\title{
Untersuchungen zur Konservierung von Körnermais mit mikrowellenunterstützter Warmlufttrocknung
}

\author{
Dissertation zur Erlangung des Doktorgrades \\ der Fakultät für Agrarwissenschaften \\ der Georg-August-Universität Göttingen
}

\author{
vorgelegt von \\ Markus Böckelmann \\ geboren in Münster
}

Göttingen, Juli 2007 
D7

Referent: Prof. Dr. Wolfgang Lücke

Korreferentin: Prof. Dr. Elke Pawelzik

Tag der mündlichen Prüfung: 19. Juli 2007 


\section{Inhaltsverzeichnis}

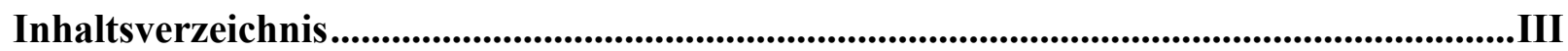

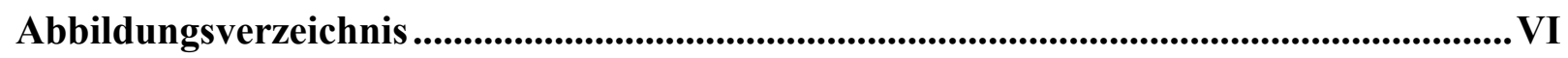

Tabellenverzeichnis ...................................................................................................................................... $\mathrm{X}$

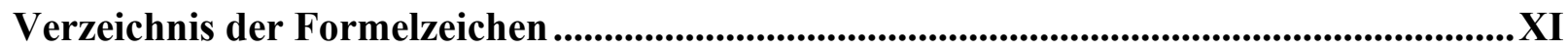

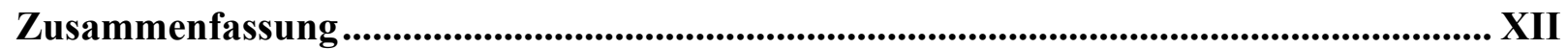

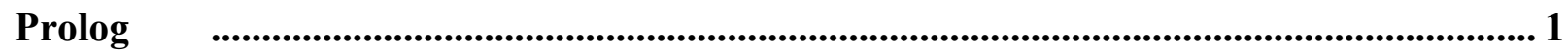

1. Teil Der Einsatz von Mikrowellen zur Maistrocknung - Grundlagen und

Laborversuche ............................................................................................................. 3

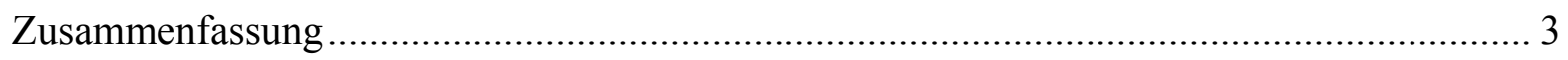

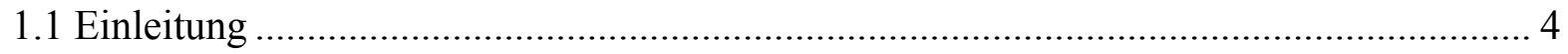

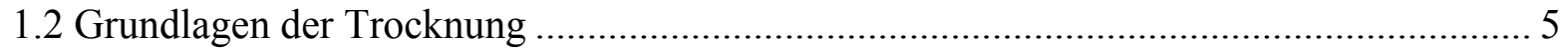

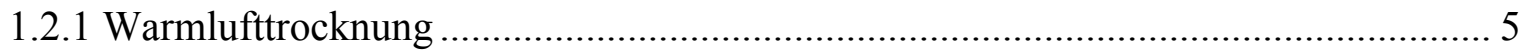

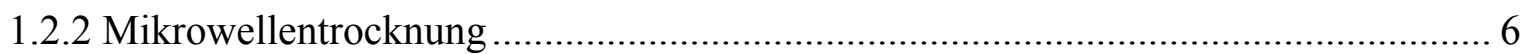

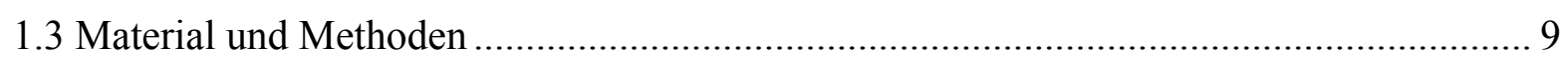

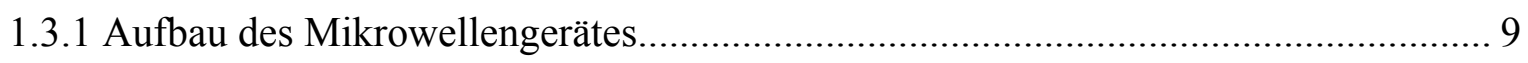

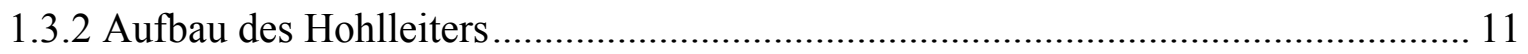

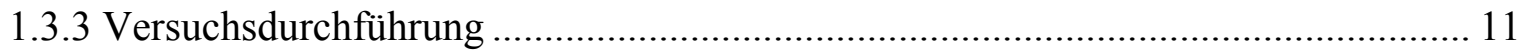

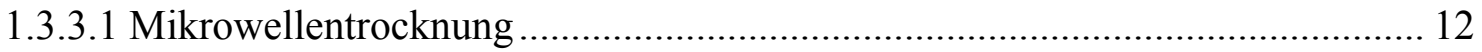

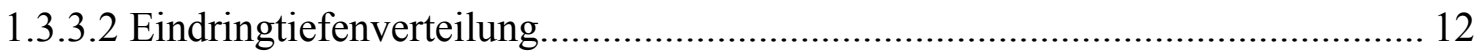

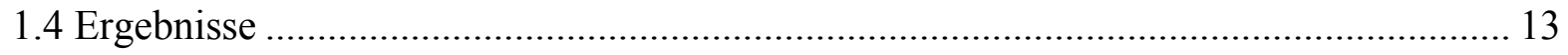

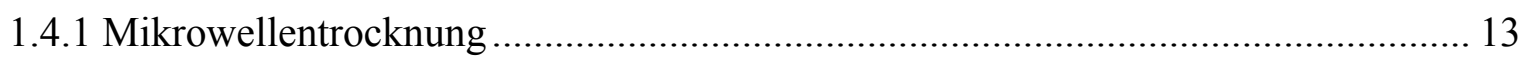

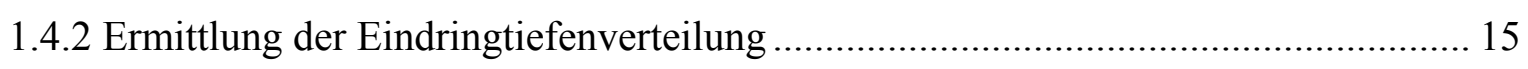

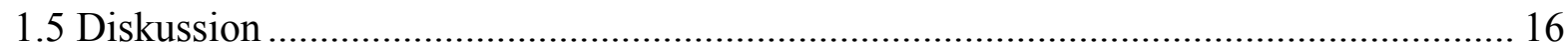

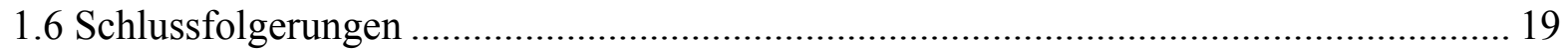

2. Teil Der Einsatz von Mikrowellen zur Maistrocknung - Anwendungen im Technikumsmaßstab ............................................................................... 21

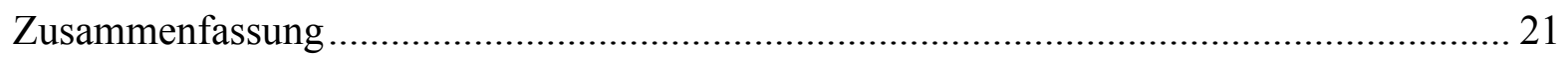

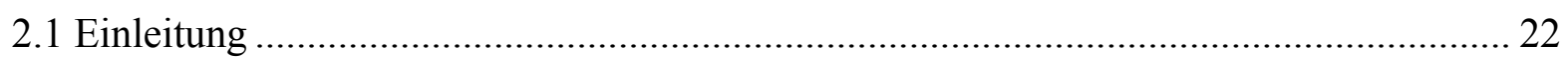

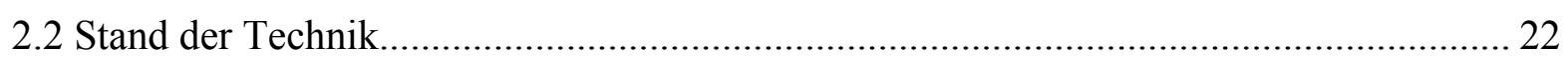

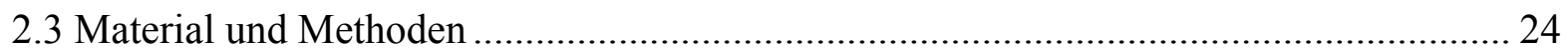

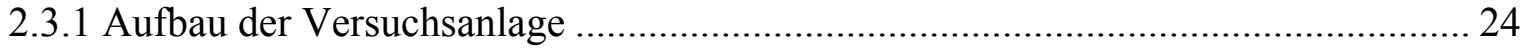

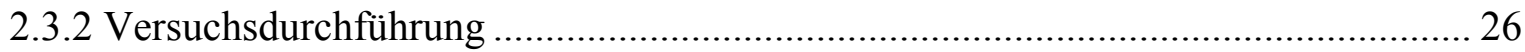

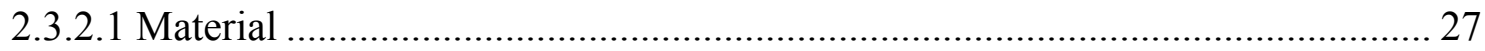


2.3.2.2 Versuch 2.1: Ermittlung des Eindringverhaltens in eine Körnermaisschicht nur mit Mikrowellenerwärmung

2.3.2.3 Versuch 2.2: Ermittlung des Trocknungsverhaltens bei alleiniger Warmlufttrocknung.....

2.3.2.4 Versuch 2.3: Ermittlung des Trocknungsverhaltens bei kombinierter Warmluft-

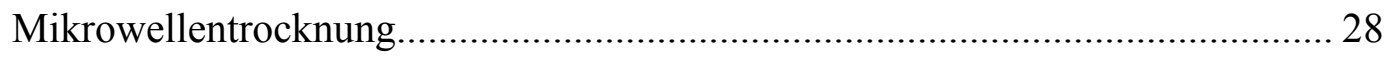

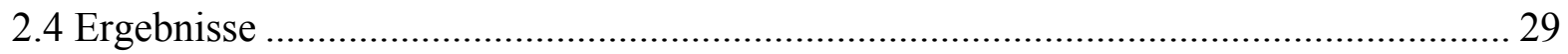

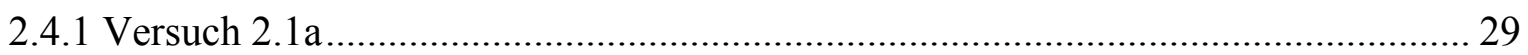

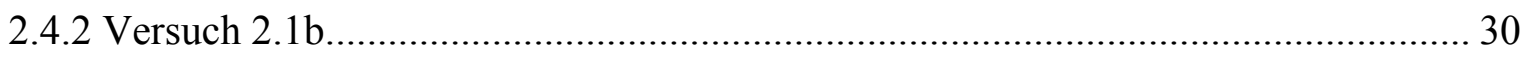

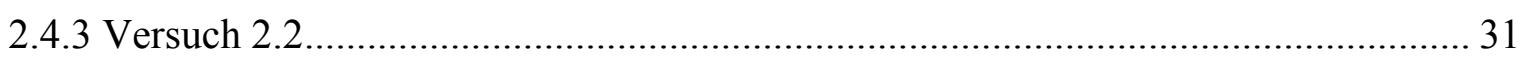

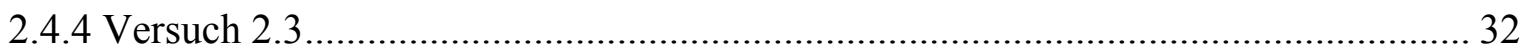

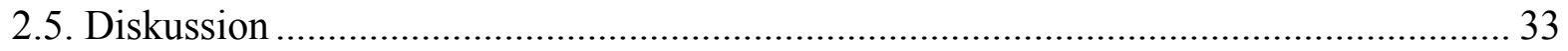

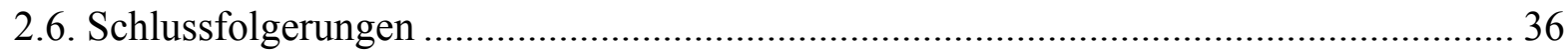

\section{Teil Einfluss von mikrowellenunterstützter Warmlufttrocknung bei der}

Körnermaistrocknung - Visualisierung mit dreidimensionaler Mikro-

Computertomographie................................................................................ 37

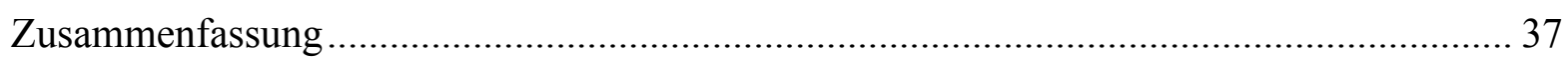

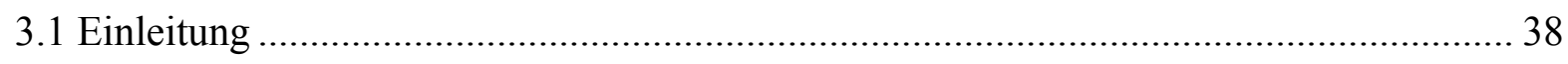

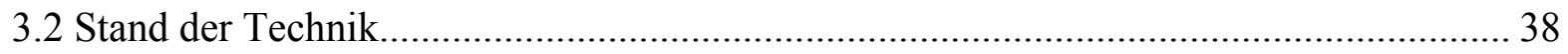

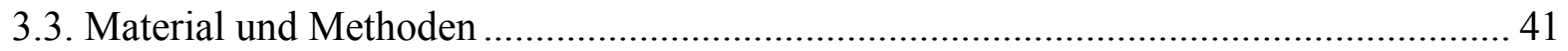

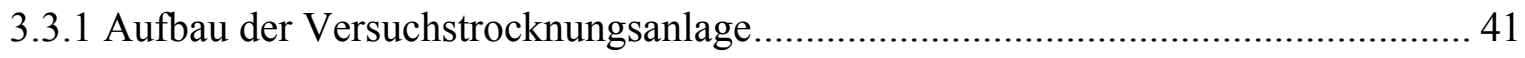

3.3.2 Aufbau der Mikrocomputertomographieanlage ................................................. 42

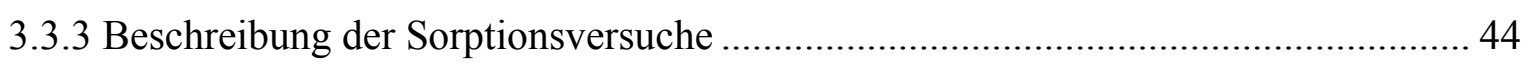

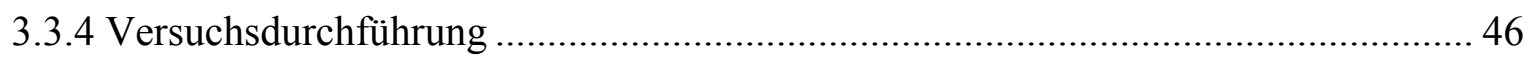

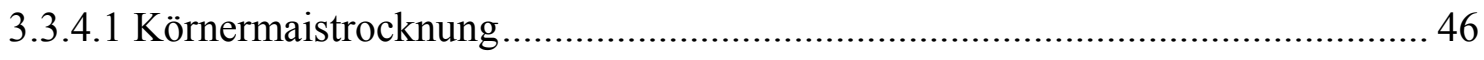

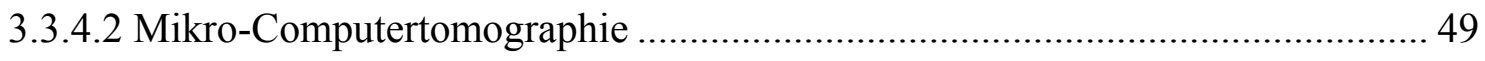

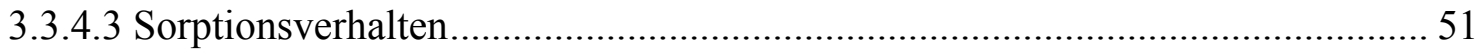

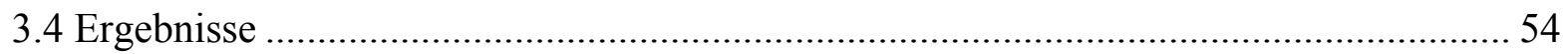

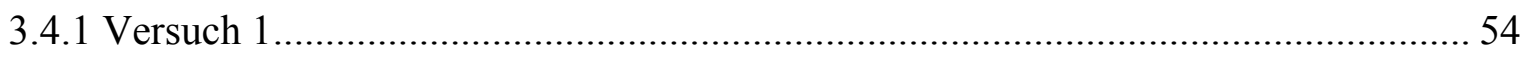

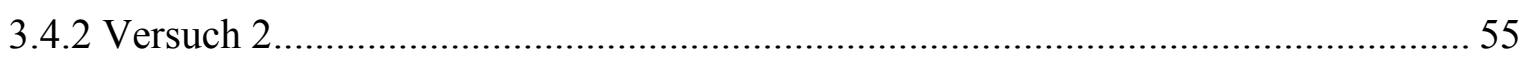

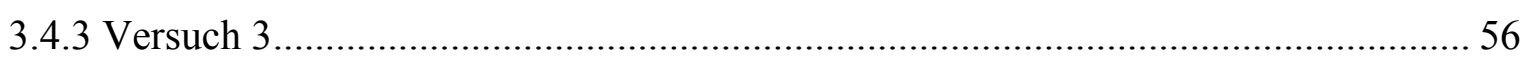

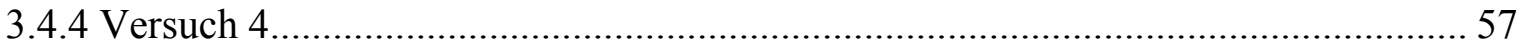

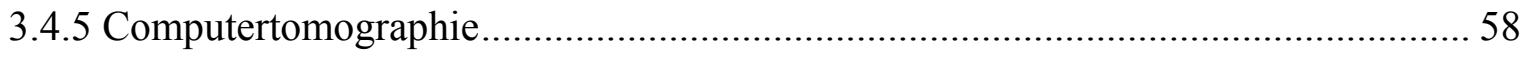

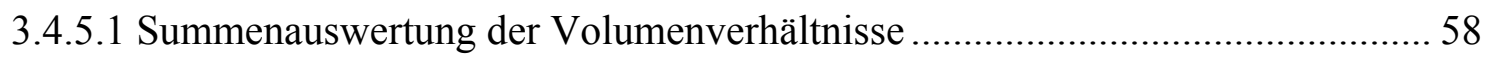

3.4.5.2 Computertomographische Aufnahmen ............................................................ 59 


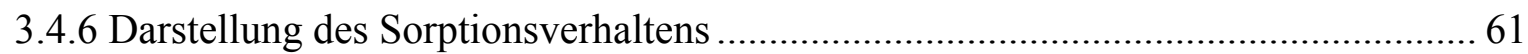

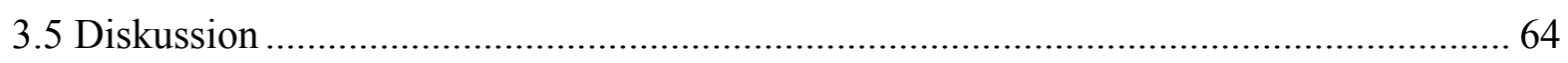

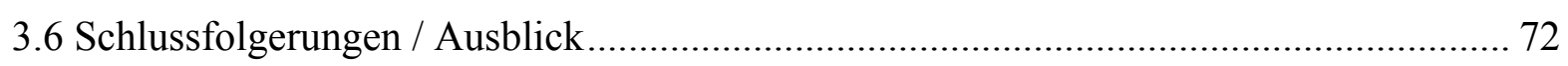

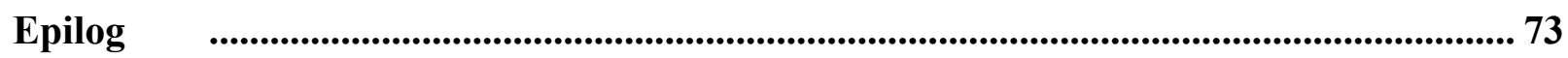

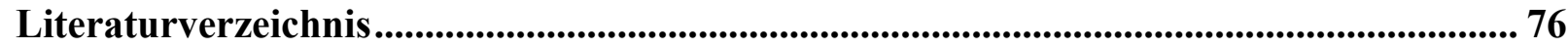

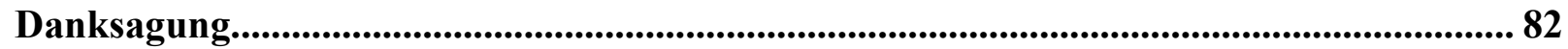

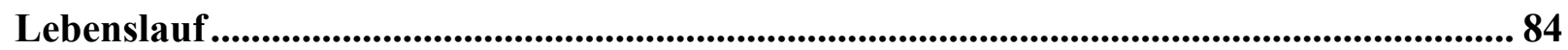




\section{Abbildungsverzeichnis}

Bild 1.1: a) Trocknungsverlauf eines kapillarporösen Gutes mit hygroskopischem Bereich

b) Charakteristischer Trocknungsverlauf von nichtporösen Stoffen

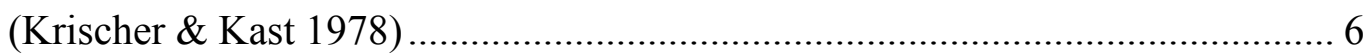

Bild 1.2: Schematische Darstellung der dielektrischen Eigenschaften von Materie in Abhängigkeit der Frequenz (Raju 2003, geändert) 7

Bild 1.3: Aufbau des Mikrowellengerätes................................................................... 10

Bild 1.4: Hohlleiter R 26

Bild 1.5: Trocknungsverlauf und Oberflächentemperaturänderung von Körnermais im Mikrowellengerät, Anfangsfeuchte 25,0 \%, Endfeuchte 14,0 \% 14

Bild 1.6: Wasserabgabe von Körnermais im Mikrowellengerät, Anfangsfeuchte 25,0\%, Endfeuchte 14,0\%

Bild 1.7: Korntemperatur in Abhängigkeit der vertikalen Position im Hohlleiter und der Applikationsdauer von Mikrowellenenergie (600 W), Anfangsfeuchte 25,0 \%. 15

Bild 1.8: Temperaturverlauf als Funktion der vertikalen Position im Hohlleiter 16

Bild 2.1: Chargenmischertrockner, Foto 25

Bild 2.2: Aufbau der Versuchsanlage 25

Bild 2.3: Ansicht in den Arbeitsraum des Chargenmischertrockners 29

Bild 2.4: Infrarotaufnahme von erntefrischem Mais im Versuchstrockner

Bild 2.5: Temperaturverteilung des durch Mikrowellen erwärmten Körnermaises. 29

Bild 2.6: Infrarotaufnahme nach $1 / 2$ Umdrehung .30 


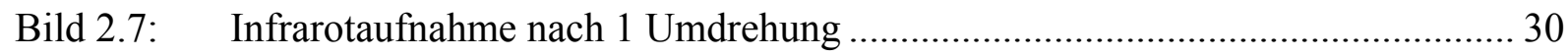

Bild 2.8: Infrarotaufnahme nach 2 Umdrehungen ...................................................... 30

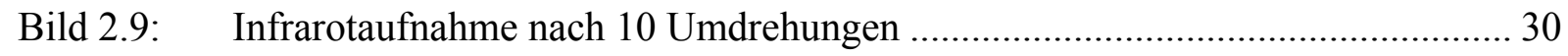

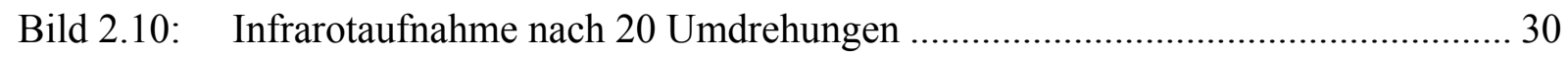

Bild 2.11: Warmlufttrocknung im Chargenmischertrockner, Diagramm ......................... 32

Bild 2.12: Mikrowellenunterstützte Warmlufttrocknung im Chargenmischertrockner,

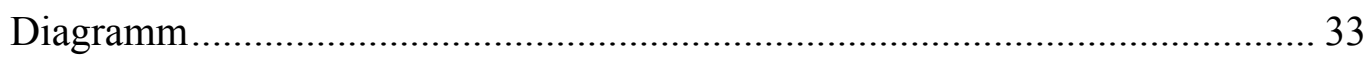

Bild 3.1: $\quad$ Abbildung Maiskorn im Schnitt (Zscheischler et al. 1990) ............................... 38

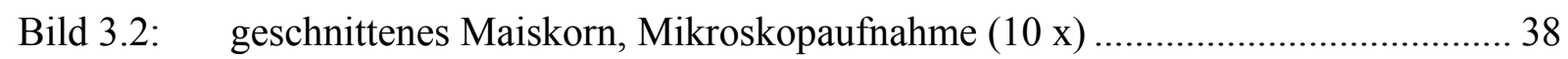

Bild 3.3: Querschnitt durch den Schalenbereich des Maiskorns (Gassner et al. 1989)...... 39

Bild 3.4: Mikroskopische Aufnahme vom Randbereich eines Maiskorns (40 x) .............. 39

Bild 3.5: Ansicht auf die CT-Röntgenkabine im Betriebszustand ................................... 42

Bild 3.6: Prinzipieller System-Aufbau der 3D-Computertomographie............................. 43

Bild 3.7: Einbettung der Maisproben in den Probeträger aus Styropor (jeweils vier Maiskörner in drei Ebenen) ....................................................... 43

Bild 3.8: Versuchsgefäß zur Ermittlung des Sorptionsverhaltens einer Körnermais-

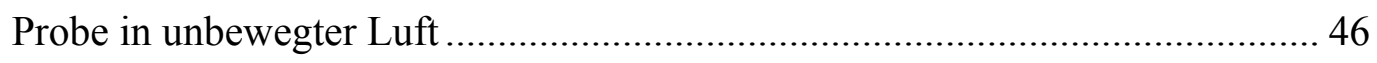

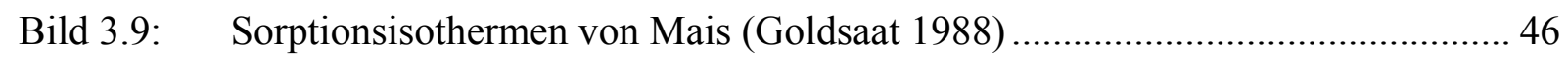

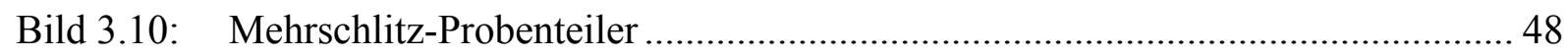


Bild 3.11: Röntgenaufnahme (linke Abbildung) eines Maiskorns in einer Schnittebene, rechtsseitig das dazugehörige segmentierte Bild

Bild 3.12: $\quad$ Übersicht Probenbenennung der Sorptionsversuche, Versuch 3.1

Bild 3.13: Körnermaisproben der Versuche 3.1 bis 3.4 in Probeträgern aus DrahtGewebe, Ansicht in den Klimaschrank 52

Bild 3.14: Körnermaisproben in Glasbehältern mit Salzlösung. Die Glasbehälter sind im Klimaschrank mit einer Innenraumtemperatur von $30^{\circ} \mathrm{C}$ aufgestellt.

Bild 3.15: Warmlufttrocknung im Chargenmischertrockner,

Ergebnisse aus Versuch 3.1 55

Bild 3.16: Mikrowellenunterstützte Warmlufttrocknung im Chargenmischertrockner, Ergebnisse aus Versuch 3.2 56

Bild 3.17: Mikrowellenunterstützte Warmlufttrocknung im Chargenmischertrockner, Ergebnisse aus Versuch 3.3. 57

Bild 3.18: Mikrowellenunterstützte Warmlufttrocknung im Chargenmischertrockner, Ergebnisse aus Versuch 3.4 58

Bild 3.19: CT-Aufnahme, dreidimensional, Kontrolle feuchtes Maiskorn $37,5 \%$ Feuchtegehalt, Hohlraumanteil 2,89\% 59

Bild 3.20: CT-Aufnahme, dreidimensional, Kontrolle Raumlufttrocknung, 14,8 \% Feuchtegehalt, Hohlraumanteil 4,45 \% 60

Bild 3.21: CT-Aufnahme, dreidimensional, Versuch 3.1, Warmlufttrocknung 14,2 \% Feuchtegehalt, Hohlraumanteil 8,89\%

Bild 3.22: CT-Aufnahme, dreidimensional, Versuch 3.2, Warmlufttrocknung + MW nach thermodynamischen Überlegungen, 14,2 \% Feuchtegehalt, Hohlraumanteil 7,74 \% 60 
Bild 3.23: CT-Aufnahme, dreidimensional, Versuch 3.3, Warmlufttrocknung + MW unterbrochen und mit reduzierter Sendeleistung $14,1 \%$ Feuchtegehalt, Hohlraumanteil 8,56\%

Bild 3.24: CT-Aufnahme, dreidimensional, Versuch 3.4, Warmlufttrocknung + MW permanent, 14,1 \% Feuchtegehalt, Hohlraumanteil 10,30\% 61

Bild 3.25: Relative Masseänderungen der Einzelproben in Sorptionslösungen Versuche 3.1a bis 3.4.d.

Bild 3.26: Wasserentzug je Trocknungsminute, Versuche 3.1 bis 3.4. 66

Bild 3.27: CT-Aufnahme, Körnermais feucht 67

Bild 3.28: CT-Aufnahme, Körnermais luftgetrocknet $\left(20^{\circ} \mathrm{C}, 65 \%\right.$ rel. Luftfeuchte. 67

Bild 3.29: Feuchtegrad und Trocknungsgeschwindigkeit aus Versuch 3.1 und 3.3, aufgetragen über der Trocknungsdauer 68

Bild 3.30: Trocknungsenergieeinsatz im Vergleich, Versuche 3.1 bis 3.4 69

Bild 3.31: CT-Schnitt, ausgewähltes Korn aus Versuch 3.4 70

Bild 3.32: CT-Schnitt, ausgewähltes Korn aus Versuch 3.1 70 


\section{Tabellenverzeichnis}

Tabelle 1.1: Für industrielle, wissenschaftliche und medizinische Anwendungen

Freigegebene Frequenzen (ISM-Frequenzen, VDE 0875 2003). 8

Tabelle 1.2: Technische Daten des Labor-Mikrowellengerätes 10

Tabelle 3.1: Relative Feuchten über gesättigten Salzlösungen (Römpp 1995) 45

Tabelle 3.2: Summenauswertung, Volumenverhältnisse der Maisproben aus Versuch

3.1 bis 3.4, feuchter und raumluftgetrockneter Körnermais

Tabelle 3.3: Kornfeuchtegehalt nach 150 Tagen Lagerdauer

$\left(15^{\circ} \mathrm{C}\right.$ Lufttemperatur und $60 \%$ rel. Luftfeuchte) 62

Tabelle 3.4: Kornfeuchtegehalt nach 48 Tagen Aufenthalt im Klimaschrank $\left(30{ }^{\circ} \mathrm{C}\right.$ Lufttemperatur und $62 \%$ rel. Luftfeuchte). 62 


\section{Verzeichnis der Formelzeichen}

\begin{tabular}{|c|c|c|}
\hline Symbol & Dimension & Bezeichnung \\
\hline $\mathrm{c}_{0}$ & $\mathrm{~m} \mathrm{~s}^{-1}$ & Lichtgeschwindigkeit $\left(3 \cdot 10^{8)}\right.$ \\
\hline$c_{p}$ & $\mathrm{Ws} \cdot(\mathrm{g} \cdot \mathrm{K})^{-1}$ & spezifische Wärme \\
\hline $\mathrm{d}$ & $\mathrm{cm}$ & Eindringtiefe \\
\hline $\mathrm{E}$ & $\mathrm{V} \cdot \mathrm{cm}^{-1}$ & elektrische Feldstärke \\
\hline $\mathrm{f}$ & $\mathrm{s}^{-1}$ & Frequenz \\
\hline $\mathrm{F}_{\mathrm{A}}$ & $\%$ & Anfangsfeuchtegehalt \\
\hline $\mathrm{F}_{\mathrm{E}}$ & $\%$ & Endfeuchtegehalt \\
\hline $\mathrm{j}$ & - & mathematischer Operator $(\sqrt{-1})$ \\
\hline$\dot{\mathrm{m}}_{\mathrm{D}}$ & $\mathrm{kg} \cdot(\mathrm{kg} \cdot \mathrm{s})^{-1}$ & Trocknungsgeschwindigkeit \\
\hline$\dot{\mathrm{m}}_{\mathrm{DI}}$ & $\mathrm{kg} \cdot(\mathrm{kg} \cdot \mathrm{s})^{-1}$ & Anfangstrocknungsgeschwindigkeit \\
\hline $\mathrm{m}_{\mathrm{H} 2 \mathrm{O}}$ & $\mathrm{kg}$ & Masse des entzogenen Wassers \\
\hline $\mathrm{m}_{\text {Frischmasse }}$ & $\mathrm{kg}$ & Masse des zu trocknenden Gutes \\
\hline $\mathrm{P}_{\mathrm{Hf}}$ & $\mathrm{W}$ & absorbierte, in Wärme umgesetzte Leistung \\
\hline $\mathrm{P}_{\text {th }}$ & $\mathrm{W}$ & Wärmeleistung \\
\hline$Q_{\text {th }}$ & Ws & thermische Energie \\
\hline $\mathrm{T}$ & ${ }^{\circ} \mathrm{C}$ & Temperatur \\
\hline$\Delta \mathrm{T}$ & ${ }^{\circ} \mathrm{C}$ & Temperaturdifferenz \\
\hline $\mathrm{t}$ & $\mathrm{s}$ & Zeit \\
\hline $\tan \delta$ & - & Verlustfaktor \\
\hline $\mathrm{t}_{\mathrm{kn} 1}, \mathrm{t}_{\mathrm{kn} 2}$ & - & Knickpunkt 1, Knickpunkt 2 \\
\hline $\mathrm{V}$ & $\mathrm{cm}^{3}$ & Volumen \\
\hline$\dot{\mathrm{V}}$ & $\mathrm{m}^{3} \cdot \mathrm{h}^{-1}$ & Volumenstrom \\
\hline$\varepsilon_{0}$ & $\mathrm{As} \cdot(\mathrm{V} \cdot \mathrm{cm})^{-1}$ & Dielektrische Feldkonstante $\left(8,85 \cdot 10^{-14}\right)$ \\
\hline$\varepsilon_{\mathrm{r}}$ & - & relative Dielektrizitätszahl \\
\hline$\varepsilon_{\mathrm{r}}, \varepsilon^{\prime}$ & - & Realteil der komplexen Permittivität \\
\hline$\varepsilon_{\mathrm{r}}{ }^{\prime}, \varepsilon^{\prime \prime}$ & - & Imaginärteil der komplexen Permittivität \\
\hline$\rho$ & $\mathrm{g} \cdot\left(\mathrm{cm}^{3}\right)^{-1}$ & Dichte \\
\hline
\end{tabular}




\section{Zusammenfassung}

Die vorliegende Monographie untersucht, wie Mikrowellen auf feuchten Körnermais wirken und unter welchen Bedingungen Mikrowellenenergie vorteilhaft zur Trocknung und Unterstützung der Warmlufttrocknung eingesetzt werden kann. Die von der Praxis geforderten Verfahrensoptimierungen zur Körnermaistrocknung orientieren sich primär an der Reduzierung des spezifischen Trocknungsenergiebedarfes, der Verkürzung thermischer Prozesswege und der Optimierung der Steuerung bzw. Regelung. Hierbei steht jedoch das Prinzip der Warmlufttrocknung weiterhin im Vordergrund. Eine Erhöhung der Trocknerleistung durch eine Warmlufttemperaturerhöhung ist aus energetischen und qualitativen Gründen begrenzt. Hohe Warmlufttemperaturen von mehr als $140{ }^{\circ} \mathrm{C}$ führen bei fortschreitender Trocknung aufgrund verringerter Produktfeuchtigkeit zu hohen Korntemperaturen. Die Trocknungsgeschwindigkeit wird dabei kaum erhöht und die Gefahr der Kornschädigung steigt. Es stellt sich daher die Frage, ob und wie der Einsatz von Mikrowellenenergie die Körnermaistrocknung beeinflusst und Mikrowellen die Trocknerleistung erhöhen können.

Im ersten Teil werden Grundlagen und Laborversuche zur Maistrocknung mit Mikrowellen beschrieben. Bei der Mikrowellentrocknung bewegen sich unter dem Einfluss eines elektrischen Wechselfeldes Dipole innerhalb eines Stoffes und erzeugen Wärme. Die Anregung der Ladungen im Molekül erfolgt entsprechend ihrer Masse in Abhängigkeit von der Frequenz. Bei hohen Frequenzen können nur Ladungsträger mit geringer Masse wie Elektronen angeregt werden, während niedrige Frequenzen alle Ladungsträger ansprechen. Die Erwärmung mit Mikrowellenenergie soll durch eine geeignete Frequenz und Applikation gleichmäßig im Produkt erfolgen, und nicht primär durch Wärmeleitung von lokalen, stark erwärmten Bereichen innerhalb des Produktes ausgehen (hot spots). Die Frequenz von 2,45 GHz hat sich bei Erwärmungsverfahren mit Mikrowellenenergie durchgesetzt. Um Aussagen zum Erwärmungsverhalten von feuchtem Körnermais mit Mikrowellenenergie zu gewinnen, sind zwei unterschiedliche Versuchsanordnungen konzipiert worden. Zunächst sind in einem für Laboruntersuchungen modifizierten Haushalts-Mikrowellengerät Trocknungsversuche durchgeführt worden. In Abhängigkeit der Applikationsdauer und Mikrowellenleistung wurde die Trocknungsdauer und damit einhergehende Oberflächentemperatur einer drei- bis vierlagigen Körnermaisprobe ermittelt.

Um eine Aussage über die Eindringtiefenverteilung in Abhängigkeit von der Applikationsdauer in eine feuchte Körnermaisschüttung zu erhalten, wurde in einem weiteren Versuch in einem 
R-26 Hohlleiter befindlicher erntefrischer Körnermais mit einem Anfangsfeuchtegehalt von $25 \%$ mit Mikrowellenenergie erwärmt. Erwartungsgemäß stellte sich an der Körnermaisoberfläche die höchste Temperatur ein, wobei im Abstand von $40 \mathrm{~cm}$ unterhalb der Körnermaisoberfläche praktisch keine Erwärmung mehr feststellbar war. Eine zu hohe Energiedichte führte bei diesem Versuch durch eine übermäßige lokale Erwärmung zu starken Bräunungen der Maiskörner. Einzelne Körnerverklumpungen zeigten eine braun-schwarze Farbe. Die Feuchtigkeitsverteilung innerhalb der Kornschüttung war ungleich. Farbliche Veränderungen wiesen auf Kornschäden hin. Es zeigte sich aus dem ersten Versuch, dass Körnermais mit einem Anfangsfeuchtegehalt von $25 \%$ sich mit einer Mikrowellenapplikation von 22 Minuten auf einen Endfeuchtegehalt von 14 \% trocknen lässt. Der zweite Versuch macht deutlich, dass sich sowohl die Eindringtiefe als auch die Temperaturerhöhung durch Mikrowellenenergie in einer unbewegten Kornschüttung ungleichmäßig ausbildet. Es stellt sich daher die Frage, welche verfahrenstechnischen Anforderungen vereint werden müssen, um die Vorteile einer kurzen Trocknungszeit durch den Einsatz von Mikrowellenenergie in Kombination mit Warmluft ausschöpfen zu können.

Aus diesem Grund wird in dem zweiten Teil der Arbeit aufgezeigt, welche Wechselwirkungen sich bei unterschiedlichen Kombinationen von Warmluft- und Mikrowellenenergieeinsatz bei der Körnermaistrocknung ausbilden. Weiter wird untersucht welche physikalisch-technischen Kriterien hinsichtlich des Trocknungsraumes erfüllt sein müssen, um eine möglichst vollständige Energieumsetzung in Wärme unter Beachtung kornphysiologischer Limits zu ermöglichen. Hierzu ist ein absätzig arbeitender Chargenmischertrockner im Technikumsmaßstab entwickelt worden. Dessen Fassungsvermögen beträgt ca. 50 Kilogramm Körnermais, wobei für die Versuche Probemengen von 25 und 30 Kilogramm gewählt worden sind. Der Versuchstrockner ist so aufgebaut, dass er hinsichtlich des Energieeintrages sowohl als reiner Warmlufttrockner und als reiner Mikrowellentrockner sowie in jeder beliebigen Kombination zu benutzen ist. Die Versuchsanlage ist mobil. Der Aufbau ist so ausgelegt, dass sowohl die Trocknungsluftbedingungen als auch die Produktbewegung während der Trocknung stufenlos verändert werden können. So ist es möglich, die Abhängigkeit der physikalisch relevanten Parameter ( $\mathrm{Zu}$ - und Abluftbedingungen, Wasserentzug, Volumenänderung, Energieeintrag usw.) während der Trocknungsversuche aufzuzeichnen.

Mit Hilfe einer Infrarotkamera wird untersucht, in welcher geometrischen Ausprägung sich Mikrowellen in der Trocknungskammer ausbilden. Auf diese Weise lassen sich wichtige Aussagen darüber machen, wie Mikrowellen in einer Maisschüttung wirken. Die 
Versuchsergebnisse zeigen, dass durch eine angepasste dielektrische Trocknung der Anteil konvektiver Energie reduziert werden kann. Im Vergleich zur reinen Warmlufttrocknung führt bei einem Parallelversuch die Verfahrenskombination mit einer mikrowellenunterstützten Warmlufttrocknung zu einer Verkürzung der Trocknungszeit um 35 Prozentpunkte und zu einer Reduzierung des Gesamtenergieeinsatzes um 22,6 Prozentpunkte. Damit die Mikrowellenenergie gleichmäßig im Produkt wirkt, ist eine ausreichende Produktbewegung während der Trocknung erforderlich. Weiterhin ist es vorteilhaft, wenn die Wirkrichtung der Mikrowellenergie mit dem Warmluftstrom gleichgerichtet ist.

Der dritte Teil der Arbeit untersucht verschiedene Proben, die im Chargenmischertrockner mit unterschiedlichen Verhältnissen von Warmluft und Mikrowellenenergie getrocknet worden sind. Hierbei wird nicht die Maisschüttung, sondern das Einzelkorn untersucht. Ziel ist es festzustellen, ob und welche physikalischen Wirkungen durch unterschiedliche Trocknungsenergieeinträge und damit verbundene unterschiedliche Trocknungsgeschwindigkeiten hervorgerufen werden. In der Arbeit wird ein neuer Ansatz vorgestellt und angewendet, um die physikalischen Vorgänge zu visualisieren, die durch Trocknung im Korn auftreten. Bisherige zweidimensionale optische mikroskopische Aufnahmen sind bei getrocknetem Maiskorn kaum anwendbar, weil Mikrotomschnitte aufgrund der Kornstruktur keinen planen Schnitt ermöglichen. Zudem gelingt dieses nicht zerstörungsfrei. Versuche $\mathrm{zu}$ durch Trocknungsenergie verursachten räumlichen Temperaturverteilung im Korn sind durch den Einsatz spezieller Mikrothermometer möglich, sie erlauben aber nur theoretisch eine grobe Beurteilung der Wirkung von Trocknungswärme auf das Korn. Die Röntgentechnik ermöglicht den zerstörungsfreien Blick in das Innere von Maiskörnern, die räumliche Zuordnung von Gewebeteilen und deren exakte Vermessung mit einer maximalen Auflösung von $10 \mu \mathrm{m}$. Anders als bei der zweidimensionalen Röntgentechnik, in der die Tiefeninformation verloren geht, erlaubt die rechnergestützte Volumenkonstruktion mit Hilfe einer rotierenden Probe erstmals einen dreidimensionalen Bildaufbau des Maiskornes. Im Vergleich zur zweidimensionalen Röntgentechnik stellen sich Unterschiede der stärker strahlenabsorbierenden Frucht- und Samenschalen aussagefähiger dar. Die kurzen Rechenzeiten für die Konstruktion der Volumina aus zweidimensionalen Schnittebenen und ein Programm für die automatische Bildauswertung ermöglichen die Röntgenanalyse, die zur dreidimensionalen Visualisierung führt. Als weltweit erstes und bislang einziges Pflanzenzuchtunternehmen hat das Unternehmen Fr. Strube GmbH \& Co. KG, Söllingen, in Zusammenarbeit mit dem Entwicklungszentrum für Röntgentechnik der Fraunhofer Gesellschaft dieses hochauflösende dreidimensionale Röntgensystem für die 
Serienuntersuchung von pelletiertem Rübensaatgut entwickelt. Im Rahmen der vorliegenden Arbeit ist dieses System für die Maiskornuntersuchung modifiziert worden. Hierbei können bis zu 12 Maiskörner gleichzeitig vermessen werden. Aufbauend auf die Versuchsergebnisse zur Warmlufttrocknung mit Mikrowellenapplikation wird untersucht, wie im Gegensatz zur Trocknung unter weitgehend natürlichen Bedingungen $\left(20^{\circ} \mathrm{C}\right.$ und $65 \%$ rel. Luftfeuchtegehalt) kornphysikalische Änderungen bei der zur Futtermaisgewinnung bewährten Warmlufttrocknung mit einer Trocknungslufttemperatur von ca. $125^{\circ} \mathrm{C}$ entstehen und welche Vorgänge deutlich werden, wenn der Trocknungsenergieeintrag durch Mikrowellen erhöht wird. Die Ergebnisse zeigen auf, dass unterschiedliche Trocknungsintensitäten zu verschiedenen geometrisch-plastischen Form- und Volumenänderungen führen, wodurch sich das Sorptionsverhalten von Körnermais ändert. 


\section{Prolog}

Eine ausreichende und flächendeckende Versorgung der Bevölkerung mit Nahrungsmitteln und Energie sind weltweit tragende Ziele der politischen und wirtschaftlichen Entscheidungsebenen. Die Landwirtschaft ist die entscheidende Säule bei der Erzeugung von Nahrungsmitteln und in zunehmenden Maß auch Energielieferant. Neben Weizen und Reis gehört Mais weltweit zu den wichtigsten Kulturpflanzen. Jedes Jahr werden weltweit mehr als 140 Millionen Hektar mit Körnermais bestellt. Davon werden jährlich ca. 600 Mio. Tonnen als Körnermais geerntet. In Deutschland wurden im Jahr 2006301.800 Hektar Körnermais angebaut und mit einem Durchschnittsertrag von ca. $8 \mathrm{t} /$ ha trockene Ware geerntet. Von dieser Erntemenge werden $22 \%$ für die menschliche Ernährung benötigt, 30 \% werden als Rohstoff in der Industrie eingesetzt und $48 \%$ der Erntemenge werden verfüttert. Die Importquote bei Körnermais in Deutschland liegt bei ca. $20 \%$.

Getreide und Körnermais werden jährlich je 1-2 Monate geerntet, getrocknet und 12 Monate als Lebensmittel, Saatgut, Futter oder nachwachsender Rohstoff genutzt. Körnermais wird in Deutschland und Mitteleuropa mit ca. $35 \%$ Wassergehalt gedroschen und ist unterhalb $14 \%$ Wassergehalt lagerfähig. Körnermais muss, um ohne Verderb lagerfähig zu sein, auf einen Kornfeuchtegehalt von weniger als $14 \%$ getrocknet werden. Geographisch und klimatisch bedingt, bewegen sich die durchschnittlichen Feuchtegehalte während der Ernte in Deutschland und vielen anderen Staaten zwischen $30 \%$ und $35 \%$ Wassergehalt. Unabhängig von witterungsbedingten Erntefeuchten, die eine dauerhafte Lagerung verhindern, provozieren zukünftig engere Erntefenster in Bezug auf Produktqualität, leistungsstarke Erntetechnik und die verwendungsorientierte Konservierung regelmäßig eine trocknungstechnische Aufbereitung in unmittelbarer Nähe der Anbaustandorte. Aktuelle Ziele der Praxis sind das Erreichen möglichst günstiger spezifischer Trocknungskosten und eine Reduzierung der Trocknungszeiten, wobei die Sekundäreffekte definierter Produktqualitäten und eine Reduzierung des Trocknungsenergieeinsatzes zunehmend wichtiger werden.

Der Energiebedarf zur Trocknung einer Tonne Körnermais beträgt bei derzeitigen Warmlufttrocknungssystemen durchschnittlich $300 \mathrm{kWh}$. Im europäischen Raum dominieren derzeit Trocknungsanlagen, deren Warmlufterzeuger mit fossiler Energie befeuert werden. Vereinzelt wird in Zentraleuropa bei einzelnen Pilotanlagen auch Trocknungsenergie eingesetzt, die beispielsweise durch Hackschnitzelfeuerung oder Wärme aus Biogasanlagen oder Dampferzeugungsanlagen bereitgestellt wird. Zur Körnermaistrocknung werden in 
Deutschland jährlich etwa 72.432.000 1 Heizöl oder äquivalente Energieträger, verbraucht. Als Heizöl entspricht diese Energiemenge einem Marktwert von ca. 36 Mio. $€$ im Jahr. Aufgrund steigender Energiepreise grenzen hohe Trocknungskosten die Wertschöpfung von Körnermais zunehmend ein. Aus Sicht der Primärproduktion umfasst die Körnermaistrocknung etwa ein Drittel der variablen Kosten bis zum lagerstabilen Zustand. Bei den variablen Trocknungskosten selbst macht der Trocknungsenergiebedarf über zwei Drittel der gesamten variablen Kosten aus. Entsprechend wichtig ist eine technisch und verfahrenstechnisch optimale Trocknung. Konventionelle Warmlufttrocknungssysteme für die Körnermaistrocknung sind technisch weitgehend ausgereift.

Im Hinblick darauf wurde in der Abteilung für Agrartechnik des Departments für Nutzpflanzenwissenschaften der Universität Göttingen und der Fachhochschule Südwestfalen, Fachbereich Agrarwirtschaft in Verbindung mit dem Labor für Mikrowellentechnik am Institut für Technologie- und Wissenstransfer, TWS, im Kreis Soest e.V. untersucht, wie sich durch

eine Verfahrenskombination aus Warmlufttrocknung und Mikrowellenapplikation Prozesse der Körnermaistrocknung optimieren lassen. Die vorliegende Monographie beschäftigt sich aus unterschiedlichen Fragestellungen mit der Anwendbarkeit von Mikrowellenenergie zur Unterstützung der Konvektionstrocknung von Körnermais. Der erste Teil gibt einen Überblick über die Grundlagen von Mikrowellentechnik und Konvektionstrocknung und stellt die Untersuchungen zur Körnermaiserwärmung mit Mikrowellenenergie im Labormaßstab vor. Im zweiten Teil werden Trocknungsversuche an Körnermais in einem eigens für dieses Forschungsvorhaben entwickelten Chargenmischertrockner vorgestellt, wobei Ergebnisse der Warmlufttrocknung mit denen der mikrowellenunterstützten Warmlufttrocknung verglichen werden. Der dritte Teil beleuchtet, wie Konvektions- und Mikrowellenenergie im Korninnern wirkt. Hierzu werden vier Trocknungsversuche mit unterschiedlichen Kombinationen des Konvektions- und Mikrowellenenergieeinsatzes durchgeführt, wobei Proben während der Trocknung entnommen werden, um mit einer Mikro-Computertomographieanlage die physikalischen Zustände zu visualisieren, die durch Trocknung im Korn entstehen. Die Ergebnisse werden anschließend mit Sorptionsversuchen verglichen. Auf Grundlage dieser Versuche lassen sich Aussagen zur Wirkung unterschiedlicher Trocknungsintensitäten auf das Trocknungsgut ableiten. 


\section{Teil}

\section{Der Einsatz von Mikrowellen zur Maistrocknung - Grundlagen und Laborversuche}

\section{Zusammenfassung}

Der Trend bei Trocknungsanlagen geht zu immer größeren Einheiten. Dabei stoßen konventionelle mit Warmluft betriebene Systeme, insbesondere solche für Körnermais, an ihre Leistungsgrenzen. Eine Unterstützung der konventionellen Trocknungssysteme durch Mikrowellenenergie ist eine Möglichkeit zur Trocknungsbeschleunigung. In dieser Ausarbeitung werden die trocknungstechnischen Grundlagen, energetische Vergleiche mit bestehenden Systemen der Körnermaistrocknung sowie die verfahrenstechnischen Möglichkeiten einer kombinierten Warmluft-Mikrowellentrocknung aufgezeigt. In Laborversuchen wird die Anwendbarkeit von Mikrowellenenergie zur Körnermaistrocknung untersucht. Die Ergebnisse bilden die Basis zur verfahrenstechnischen Dimensionierung einer kombinierten Warmluft-Mikrowellentrocknung von Körnermais im Technikumsmaßstab. 


\subsection{Einleitung}

In Körnermaisanbaugebieten müssen kurzfristig hohe anfallende Körnermaismengen konserviert werden. Durchschnittlich steigt im Jahresvergleich in Mitteleuropa der installierte Trocknerdurchsatz von Neuanlagen um ca. $0,2 \mathrm{t} \cdot \mathrm{h}^{-1}$ bei einer Entfeuchtung von $35 \%$ auf $15 \%$. Die Druschkapazität der Mähdrescher erhöht sich durchschnittlich um $0,5 \mathrm{t} \cdot \mathrm{h}^{-1} \mathrm{jährlich}$ (Rademacher 2003, Böckelmann 2003). Damit besteht zwischen der Drescherkapazität und der Trocknerleistung in der Praxis eine immer größer werdende Diskrepanz.

Die von der Praxis geforderten Verfahrensoptimierungen zur Körnermaistrocknung orientieren sich primär an der Reduzierung des spezifischen Trocknungsenergiebedarfes, Verkürzung der thermischen Prozesswege und Optimierung der Steuerung. Hierbei steht jedoch das Prinzip der Warmlufttrocknung weiterhin im Vordergrund (Gehrmann 2003).

Aus der internationalen Literatur sind Veröffentlichungen bekannt, die den Einsatz von Mikrowellenenergie zur Trocknung sowie zur Feuchtebestimmung (Mohsenin 1981) landwirtschaftlicher Produkte beschreiben. In seinen Versuchen zur Mikrowellenapplikation an Möhrenscheiben hat Tarsa (1991) festgestellt, dass es aus ökonomischen Gründen angebracht ist, das leicht entfernbare Wasser durch Konvektionstrocknung zu entziehen und erst später mit der Mikrowellenapplikation zu beginnen. Aufgrund der niedrigen Wassergehalte zum Ende der Trocknung erfolgt eine schlechte Umsetzung der Mikrowellenenergie in Wärme. Die Endtrocknung ist vorteilhaft mit Konvektionsenergie abzuschließen. Sowohl aus den theoretischen Betrachtungen als auch an Trocknungsversuchen mit Kartoffelscheiben kam Lücke (1992) zu dem Ergebnis, dass ergänzend zur Warmlufttrocknung eine Mikrowellenapplikation dann vorteilhaft wirkt, wenn die Einkopplung der Mikrowellenenergie sowohl konstruktiv-technisch als auch im Sinne des Trocknungsprofils aufeinander abgestimmt ist. Erste Versuche zur mikrowellenunterstützten Warmlufttrocknung von Hopfen kommen zu dem Ergebnis, dass bei einem Mikrowellenenergieeinsatz von 3,3 kWh/kg Trockenhopfen die Trocknungsleistung auf das 9-fache gesteigert werden kann. Vorteilhaft ist eine Kombination aus Warmluft- und Mikrowellentrocknung, wobei der Einsatzzeitpunkt und die Einsatzdauer produktabhängig erfolgen (Münsterer \& Heindl 2000). In ihren Untersuchungen zur mikrowellenunterstützten Trocknung von Arznei- und Gewürzpflanzen kommen Heindl \& Müller (2002) zu dem Ergebnis, dass die Trocknungszeit je nach Produkt um $50 \%$ bis $90 \%$ reduziert werden kann. Hierbei lässt sich fallweise Farbe, Form oder Gehalt an wertgebenden Inhaltsstoffen verbessern. 
In einer Ausarbeitung von Gunasekaran (1990) wird die Trocknung von Körnermaisproben mit Mikrowellen dahingehend untersucht, welche Pulsraten zum geringsten Energiebedarf führen. Dabei werden die sich einstellenden Produkttemperaturen nicht aufgeführt. Diese sind jedoch für die Trocknung ein wichtiges Kriterium. Die dielektrischen Eigenschaften von Körnermais haben Trabelsi \& Nelson (2003) in einem Feuchtebereich zwischen $13 \%$ bis $20 \%$ und einer Korntemperatur von $23{ }^{\circ} \mathrm{C}$ durch Messungen bestimmt. Der Anfangsfeuchtegehalt ist bei der Maistrocknung in der Regel aber höher. Konstruktive Reserven sind bei Konvektionstrocknungsverfahren für Körnermais weitgehend ausgeschöpft. Eine Erhöhung der Trocknerleistung durch eine Warmlufttemperaturerhöhung ist aus energetischen und qualitativen Gründen begrenzt. Hohe Warmlufttemperaturen von mehr als $140{ }^{\circ} \mathrm{C}$ führen bei fortschreitender Trocknung aufgrund verringerter Produktfeuchtigkeit $\mathrm{zu}$ hohen Korntemperaturen. Die Trocknungsgeschwindigkeit wird dabei kaum erhöht und die Gefahr der Schädigung steigt (Mühlbauer 1980). Es stellt sich daher die Frage, ob und wie der Einsatz von Mikrowellenenergie die Körnermaistrocknung beeinflusst.

\subsection{Grundlagen der Trocknung}

\subsubsection{Warmlufttrocknung}

Konvektionstrockner erfordern eine aufeinander abgestimmte Prozessluftmenge, Heizleistung und Verweildauer des Trocknungsgutes. Für die Konvektionstrocknung kapillarporöser, hygroskopischer Stoffe soll auf das Modell von Krischer \& Kast (1978) zurückgegriffen werden. Der Trocknungsverlauf (Abbildung 1a) bei kapillarporösen Stoffen ist durch drei Abschnitte charakterisiert. Im ersten Abschnitt wird die Oberfläche des Gutes getrocknet bis zum ersten Knickpunkt $\left(\mathrm{t}_{\mathrm{kn} 1}\right)$. Er ist gekennzeichnet durch eine konstante Trocknungsgeschwindigkeit. Im zweiten und dritten Abschnitt wird kapillar und osmotisch gebundenes Wasser abgeführt. Die Trocknungsfront dringt in das Gut ein, bis schließlich das hygroskopische Gleichgewicht erreicht wird. Dabei verlängern sich ständig die Diffusionswege bei gleichzeitig steigendem Widerstand für den Wärmeeintrag. Dies führt zu einer ständig sinkenden Trocknungsgeschwindigkeit mit einem weiteren Knickpunkt $\left(t_{\mathrm{kn} 2}\right)$, der sich durch den Anstieg der Bindungskräfte bei niedrigeren Feuchtegraden erklären lässt (Krischer \& Kast 1978). 
a)

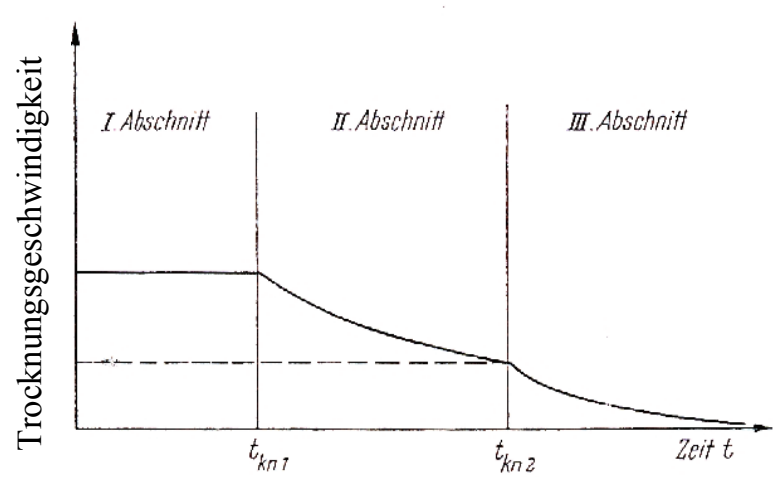

b)

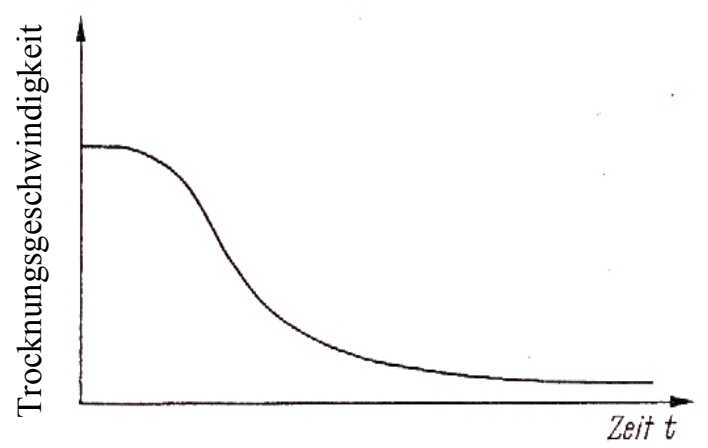

Bild 1.1: a) Trocknungsverlauf eines kapillarporösen Gutes mit hygroskopischem Bereich

b) Charakteristischer Trocknungsverlauf von nichtporösen Stoffen (Krischer \& Kast 1978)

Im Vergleich dazu zeigt Abbildung 1.1b den Trocknungsverlauf bei nichtporösen Stoffen (z.B. Gele). Im Gutsinnern erfolgt die Feuchtebewegung nur durch Flüssigkeitsdiffusion und nicht durch Dampfdiffusion. Hierdurch gibt es keine Zonen im Gut oder Zeitabschnitte, in denen verschiedene Gesetzmäßigkeiten maßgeblich sind. Die Feuchteabgabe durch Verdunstung findet nur an der Oberfläche statt (Krischer \& Kast 1978).

\subsubsection{Mikrowellentrocknung}

Unter dem Einfluss eines elektrischen Wechselfeldes bewegen sich die Dipole innerhalb eines Stoffes und erzeugen Wärme. Die Anregung der Ladungen im Molekül erfolgt entsprechend ihrer Masse in Abhängigkeit von der Frequenz. Bei hohen Frequenzen können nur Ladungsträger mit geringer Masse wie Elektronen angeregt werden, während niedrige Frequenzen alle Ladungsträger ansprechen. Mikrowellenanwendungen bewirken eine Orientierungspolarisation und liegen im Frequenzbereich von $10^{8}$ bis $10^{11} \mathrm{~Hz}$. Die wichtigsten Vorgänge sind in Bild 1.2 dargestellt. 


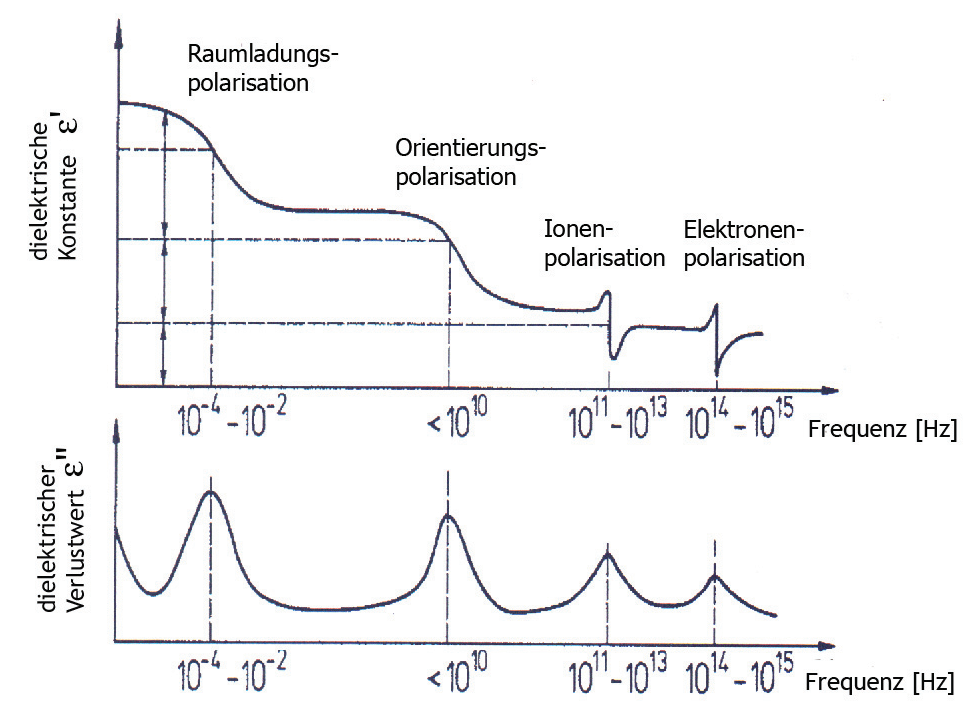

Bild 1.2: Schematische Darstellung der dielektrischen Eigenschaften von Materie in Abhängigkeit der Frequenz (Raju 2003, geändert)

Der Kurvenzug $\varepsilon^{\prime \prime}$ stellt die Absorption von Mikrowellenenergie in Materie schematisch dar. Die Kurve $\varepsilon^{\prime}$ ist ein Maß für die Polarisierbarkeit des Materials als Funktion der Frequenz. Die Angaben „Raumladungs-, Orientierungs-, Ionen und Elektronenpolarisation“ entsprechen den Frequenzbereichen, mit denen sich Ladungen von Molekülen anregen lassen. Die Frequenz, bei $\operatorname{der} \varepsilon^{\prime \prime}$ sein Maximum erreicht, wird als Relaxationsfrequenz bezeichnet. Die maximale Absorptionsfrequenz von Wasser liegt bei $\mathrm{f}=22 \mathrm{GHz}$, wobei die Eindringtiefe sehr gering ist. Deshalb ist auch aus trocknungstechnischer Sicht die verwendete Mikrowellenfrequenz von $\mathrm{f}=$ 2,45 GHz besser geeignet, weil sie eine größere Eindringtiefe ermöglicht. Die dielektrischen Eigenschaften von Stoffen werden durch die komplexe Permittivitätszahl (Püschner 1964) beschrieben. Es gilt:

$$
\varepsilon=\varepsilon_{0} \cdot \varepsilon_{\mathrm{r}}=\varepsilon_{0} \cdot\left(\varepsilon_{\mathrm{r}}{ }^{\prime}-\mathrm{j} \varepsilon_{\mathrm{r}}{ }^{\prime}\right)
$$

und daraus abgeleitet ergibt sich der Verlustwinkel: $\quad \tan \delta=\frac{\varepsilon_{\mathrm{r}}^{\prime \prime}}{\varepsilon_{\mathrm{r}}^{\prime}}$

Die frequenz - und temperaturabhängige Permittivitätszahl ist für Wasser und weitere Stoffe ermittelt (Askeland 1996, Metaxas 1983, Sembery 1989, Tabelsi \& Nelson 2003). Die Absorption von Mikrowellenenergie wird mit folgender Gleichung charakterisiert:

$$
\mathrm{P}_{\mathrm{Hf}}=\mathrm{E}^{2} \cdot 2 \cdot \pi \cdot f \cdot \varepsilon_{0} \cdot \varepsilon_{\mathrm{r}}{ }^{\prime \prime} \cdot \mathrm{V}=\mathrm{E}^{2} \cdot 2 \cdot \pi \cdot \mathrm{f} \cdot \varepsilon_{0} \cdot \varepsilon_{\mathrm{r}}{ }^{\prime} \tan \delta \cdot \mathrm{V}
$$


Mikrowellen haben die Eigenschaft, in Stoffe einzudringen. Die absorbierte Mikrowellenenergie wird in dem betrachteten Volumen V vollständig in Wärme umgewandelt. Die sogenannte Eindringtiefe ist die Tiefe, bei der die an der Produktoberfläche ankommende Leistung im Produktinnern auf den 1/e-ten Teil (ca. $37 \%$ ) gesunken ist. Sie ist von der Frequenz und den dielektrischen Eigenschaften nach Gleichung 1 und 2 abhängig (Püschner, 1964).

$$
\mathrm{d}=\frac{\mathrm{c}_{0}}{2 \cdot \pi \cdot \mathrm{f}} \cdot\left(\frac{1}{2 \cdot \varepsilon_{r}^{\prime} \cdot\left[\left(1+\left(\frac{\varepsilon_{r}^{\prime \prime}}{\varepsilon_{r}^{\prime}}\right)^{2}\right)^{0,5}-1\right]}\right)^{0,5}
$$

Um eine möglichst hohe Mikrowellenleistung zur Wärmeerzeugung in das Produkt einzubringen, sind entsprechend der Gleichung 3 eine hohe Frequenz nahe der Relaxationsfrequenz und eine hohe Feldstärke erforderlich. Eine höher gewählte Frequenz führt zu einer Verringerung der Eindringtiefe und damit zu hohen Temperaturen und einer eventuellen Überhitzung des Stoffes an der Oberfläche. Die Erwärmung mit Mikrowellenenergie soll durch eine geeignete Frequenz gleichmäßig im Produkt erfolgen, und nicht primär durch Wärmeleitung von lokalen, stark erwärmten Bereichen innerhalb des Produktes ausgehen (hot spots).

Tabelle 1.1: Für industrielle, wissenschaftliche und medizinische Anwendungen Freigegebene Frequenzen (ISM-Frequenzen, VDE 0875 2003)

\begin{tabular}{lll}
\hline $\begin{array}{l}\text { Frequenz } \\
{[\mathrm{MHz}]}\end{array}$ & $\begin{array}{l}\text { Wellenlänge } \\
{[\mathrm{m}]}\end{array}$ & $\begin{array}{c}\text { Toleranz } \\
+/-[\%]\end{array}$ \\
\hline $\begin{array}{l}\text { Hochfrequenz } \\
13,56\end{array}$ & 22,120 & 0,05 \\
27,12 & 11,060 & 0,60 \\
40,68 & 7,375 & 0,05 \\
& & \\
Mikrowelle & & \\
433,92 & 0,6914 & 0,2 \\
461,04 & 0,6507 & 0,2 \\
493,92 & 0,6074 & 0,2 \\
915,00 & 0,3279 & 2,73 \\
2450,00 & 0,1224 & 2,04 \\
5800,00 & 0,05172 & 1,29 \\
$17850,00-18150,00$ & $0,0168-0,01652$ & \\
22125,00 & 0,001356 & 0,56 \\
\hline
\end{tabular}


Hochfrequenzanwender dürfen bestimmte ISM-Frequenzen für industrielle, wissenschaftliche und medizinische Anwendungen verwenden (siehe Tabelle 1.1). Für Fernsehtechnik, Radio und andere Funksysteme sind ähnliche Frequenzbereiche freigegeben. Die Frequenz von 2,45 GHz hat sich bei Erwärmungsverfahren mit Mikrowellenenergie durchgesetzt.

Nach den Maßgaben der Thermodynamik folgt unter Berücksichtigung der thermischen Stoffeigenschaften (von Hippel 1954):

$$
\mathrm{Q}_{\mathrm{th}}=\mathrm{P}_{\mathrm{th}} \cdot \mathrm{t}=\mathrm{c}_{\mathrm{p}} \cdot \rho \cdot \mathrm{T} \cdot \mathrm{V} \quad[\mathrm{Ws}]
$$

Mit dem Ansatz $\mathrm{P}_{\mathrm{Hf}}=\mathrm{P}_{\text {th }}$ gilt für die Temperaturerhöhung je Zeit folgende Gleichung:

$$
\frac{\mathrm{dT}}{\mathrm{dt}}=\mathrm{E}^{2} \cdot \mathrm{f} \cdot\left(\frac{\varepsilon_{\mathrm{r}}^{\prime \prime}}{\mathrm{c}_{\mathrm{p}} \cdot \rho}\right)
$$

Die Temperaturerhöhung ist von dem Quadrat der Feldstärke E, der Frequenz f, dem dielektrischen Verlustwert $\varepsilon_{\mathrm{r}}{ }^{\prime \prime}$ sowie den thermischen Stoffwerten $c_{\mathrm{p}}$ und $\rho$ abhängig.

\subsection{Material und Methoden}

\subsubsection{Aufbau des Mikrowellengerätes}

Die Untersuchungen sind in einem für Laboruntersuchungen modifizierten Haushalts Mikrowellengerät (Bild 1.3) durchgeführt worden. Die in dem Arbeitsraum vorhandene Umluftheizung und Infrarotheizung wurde für Trocknungsversuche um eine Durchluftheizung ergänzt. Ein an einer Seitenwand angebrachtes Rohr enthält eine Heizung mit Gebläse, an der gegenüberliegenden Seite des Arbeitsraumes über die Raumdiagonale liegt die Austrittsöffnung. Der Luftvolumenstrom kann durch Regelung der Gebläsedrehzahl und die Lufttemperatur durch thermostatgesteuerte Heizwiderstände getrennt voneinander eingestellt werden. Bei eingeschalteter Mikrowelle bewirkt ein motorisch angetriebener Feldrührer, dass sich keine stehenden Mikrowellen ausbilden können. Auf diese Weise wird eine ungleiche Produkterwärmung durch hot spots vermieden. Derselbe Effekt ließe sich auch erreichen, indem die Probe kontinuierlich bewegt wird. 


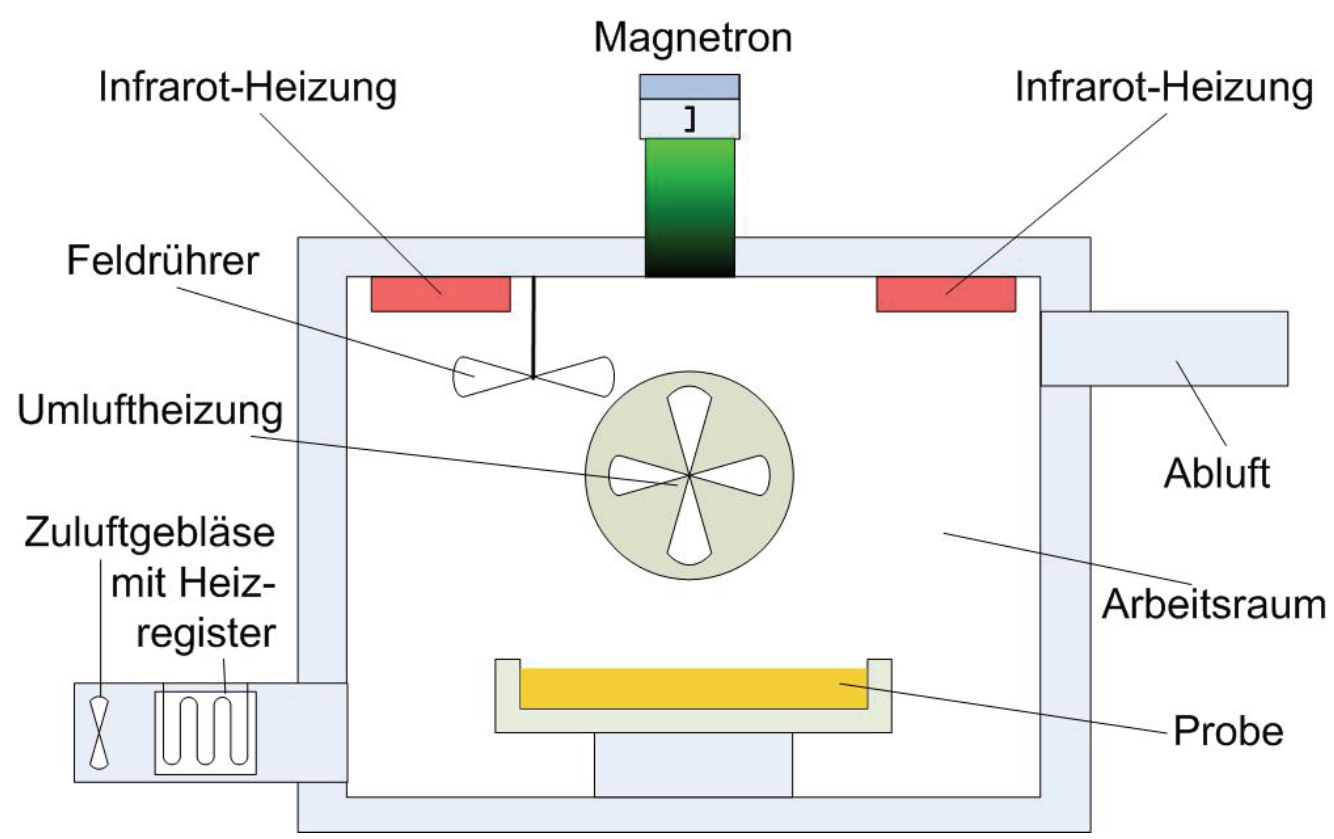

Bild 1.3: Aufbau des Mikrowellengerätes

Tabelle 1.2: Technische Daten des Labor-Mikrowellengerätes

\begin{tabular}{ll}
\hline Netzanschluss & $3 \times 400 \mathrm{~V}, 16 \mathrm{~A}$ \\
\hline Magnetron & $2 \mathrm{M} \mathrm{137}$, einstellbare Hochfrequenzleistung $120-1200 \mathrm{~W}$ \\
$\begin{array}{l}\text { Innere } \\
\text { Arbeitsraum }\end{array}$ & $\mathrm{L} \times \mathrm{T} \times \mathrm{H}=45 \times 38 \times 32 \mathrm{~cm} \quad\left(\right.$ Volumen $\left.=0,05 \mathrm{~m}^{3}\right)$ \\
Infrarot-Heizung & $\mathrm{P}=2 \times 2000 \mathrm{~W}$ \\
Umluft-Heizung & $\mathrm{P}=4000 \mathrm{~W}$ \\
Durchluft-Heizung & $\mathrm{P}=2000 \mathrm{~W}$ \\
Durchluftvolumenstrom & $\dot{\mathrm{V}}=2-35 \mathrm{~m}^{3} \mathrm{~h}^{-1}$ \\
\hline
\end{tabular}

Bei Trocknungsversuchen größeren Umfanges sind quantitative Angaben zur ausgetragenen Wassermasse erforderlich. Deshalb sind am Einlass und Auslass der Durchluftrohre Sensoren zur Erfassung der relativen Feuchte und Temperatur angebracht. Die Energieversorgung des Magnetrons ist anodenstromgeregelt. Mit einem Potentiometer kann die Leistung zwischen 120 und $1200 \mathrm{~W}$ eingestellt werden. 


\subsubsection{Aufbau des Hohlleiters}

Für die Untersuchungen zur Eindringtiefenverteilung ist ein $100 \mathrm{~cm}$ langer, genormter R 26 Hohlleiter verwendet worden. Das aufgesetzte Magnetron ist baugleich mit dem im LaborMikrowellengerät verwendeten Magnetron. Für den elektrischen Betrieb wird der aus dem Versuch mit dem Mikrowellengerät vorhandene Steuerschrank benutzt. Um einen Mikrowellen- und Produktaustritt durch den serienmäßig vorhandenen Messschlitz zu unterbinden, ist dieser über die gesamte Schlitzöffnung mit einem Edelstahllochblech mit einer Lochweite von 1,5 mm vollständig überdeckt worden.

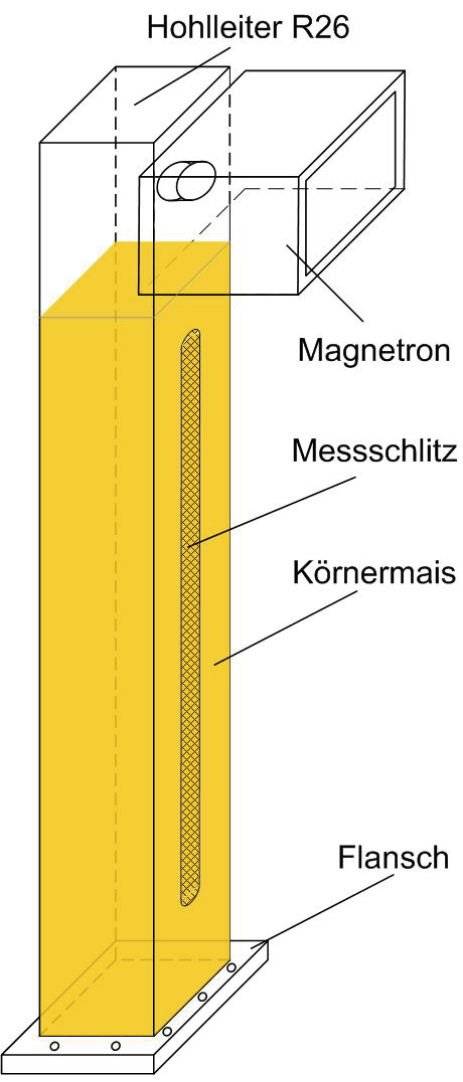

Bild 1.4: Hohlleiter R 26

\subsubsection{Versuchsdurchführung}

Der Anfangsfeuchtegehalt wurde mit einem nach kapazitivem Messverfahren arbeitendem Schnellfeuchtebestimmer für Körnermais (Pfeuffer HE 90) festgestellt und nach 24stündiger Trocknung im Trockenschrank bei $105{ }^{\circ} \mathrm{C}$ (Probe unzerkleinert) abgeglichen. Der Anfangsfeuchtegehalt betrug $25 \%$. Die in den Arbeitsraum abgegebene Mikrowellenleistung wurde auf $600 \mathrm{~W}$ eingestellt. Unmittelbar vor Versuchsbeginn wurden die Raumluftbedingungen im Labor (Raumtemperatur und Luftfeuchtegehalt) und produktbezogene Messgrößen (Uhrzeit, Oberflächentemperatur, Korntemperatur, Masse und der errechnete Produktfeuchtegehalt) aufgenommen.

Zur Messung der Oberflächentemperatur der Maiskornschüttung wurde ein berührungslos arbeitendes Infrarot-Thermometer eingesetzt. Um geometrische Oberflächenunregelmäßigkeiten im Sinne einer Messebene und auch mögliche Unterschiede der Oberflächentemperatur einzelner Maiskörner besser auszugleichen, wurde aus jeweils fünf Messstellen ein Durchschnittswert gebildet. Im Unterschied zur Oberflächentemperaturmessung wurde die Korntemperatur innerhalb der Maiskornschüttung mit einem Sekundenthermometer gemessen. Praktisch lag die Messstelle zur Erfassung der Korntemperatur zwischen den sich berührenden Maiskörnern. Es ist näherungsweise davon 
auszugehen, dass durch den Wärme- und Feuchtetransport bedingt, die mittlere Korntemperatur der Temperatur zwischen den Körnern entspricht (Mühlbauer 1972).

\subsubsection{Mikrowellentrocknung}

In einem mikrowellengeeigneten Glasgefäß wurden 500 g Körnermais gefüllt und so verteilt, dass sich eine Schichthöhe von ca. 1,5 cm einstellte. Die Trocknungsversuche wurden beendet, nachdem mittels der Duval'schen Formel der notwendige Wasserentzug - ausgehend von der Anfangsfeuchte - bestimmt worden ist, um den Endfeuchtegehalt von ca. $14 \%$ zu erreichen. Die Verwendung der Duval'schen Gleichung setzt voraus, dass der Anfangsfeuchtegehalt bekannt ist.

$$
\mathrm{m}_{\mathrm{H} 2 \mathrm{O}}=\mathrm{m}_{\text {Frischmasse }} \cdot \frac{\mathrm{F}_{\mathrm{A}}-\mathrm{F}_{\mathrm{E}}}{100-\mathrm{F}_{\mathrm{E}}}
$$

Während der Versuchsdurchführung war es somit möglich, durch Wiegen der Probe auf den vorhandenen Feuchtegehalt zu schließen. Das Probengewicht wurde mit einer Waage nach jedem Trocknungsintervall bestimmt. Die Oberflächentemperatur wurde nach jedem Trocknungsintervall (Mikrowellenzufuhr von einer Minute) außerhalb des Arbeitsraumes gemessen. Ebenso wurde nach jedem Trocknungsintervall das Gewicht ermittelt. Die Gewichtsdifferenz stellte den Wasserentzug dar. Ab der 16. Applikationsminute wurde in Zwei-Minuten Intervallen appliziert mit der Zielsetzung, die Trocknungsgeschwindigkeit nicht zu stark abfallen zu lassen. Anschließend wurde der Körnermais an Umgebungsluft gerührt. Hierdurch wurde während der Zwischenapplikationszeit außerhalb des Arbeitsraumes die Feuchteabgabe verbessert. Hiernach wurde erneut das Gewicht ermittelt und die Oberflächentemperatur gemessen. Zusätzlich wurde die mittlere Korntemperatur im Innern der Schüttung mit einem Sekundenthermometer ermittelt. Die Zeit zwischen den Mikrowellenapplikationen betrug durchgehend zwei Minuten.

\subsubsection{Eindringtiefenverteilung}

In einem weiteren Versuch wurde eine feuchte Körnermaisschüttung in einem R 26- Hohlleiter mit Mikrowellenenergie beaufschlagt. Damit sollte die Temperaturverteilung und somit das Eindringverhalten von Mikrowellenenergie in das Feuchtegut innerhalb eines Mikrowellengerätes ermittelt werden. Die Länge des Hohlleiters betrug $100 \mathrm{~cm}$, welches der rechnerischen Bemessung entsprechend etwa dem zweifachen der möglichen Eindringtiefe entsprach. Die Ermittlung der Eindringtiefenverteilung von Mikrowellenenergie in Mais lässt 
sich aufgrund einer inhomogenen Feuchteverteilung und nicht vollständig erfassbarer Korngeometrien im gesamten Maisvolumen nur bedingt bestimmen. Durch die Mikrowelleneinwirkung wurde der Körnermais erwärmt. Eine mögliche Unterstützung zur Wärmeverteilung oder Feuchteabfuhr durch bewegte Trocknungsluft erfolgte nicht. Die Temperatur des Körnermaises wurde mit einem Messintervall von 60 Sekunden und am Magnetron beginnend in Abständen von jeweils $5 \mathrm{~cm}$ in Längsrichtung des Hohlleiters durch den Messschlitz mit einem Einstich-Sekundenthermometer gemessen. Während der Temperaturmessung war das Magnetron abgeschaltet. Der Versuch wurde beendet, nachdem an der Körnermaisoberfläche durch die Mikrowellenapplikation verbrannte bzw. aufgeplatzte Maiskörner zu beobachten waren. Der Anfangsfeuchtegehalt der Körnermaisprobe betrug $25 \%$, aufgrund der kurzzeitigen Erwärmung ließen sich gleichmäßige Endfeuchtegehalte nicht bestimmen. Die Ermittlung der Eindringtiefenverteilung war erforderlich, um die mögliche Schichtdicke und die sich daraus ergebende Temperaturverteilung im Trockengut beurteilen zu können.

\subsection{Ergebnisse}

\subsubsection{Mikrowellentrocknung}

Die Trocknung von Körnermais ausschließlich mit Mikrowellenenergie zeigt einen typischen Trocknungsverlauf von kapillarporösen Stoffen (Bild 1.5). Der Wassergehalt nimmt nach einer Aufwärmphase während des Versuchs gleichmäßig ab. Die Oberflächentemperatur bleibt zunächst annähernd konstant, um dann ab einem bestimmten Wassergehalt nach der 14 . Applikationsminute deutlich anzusteigen. 


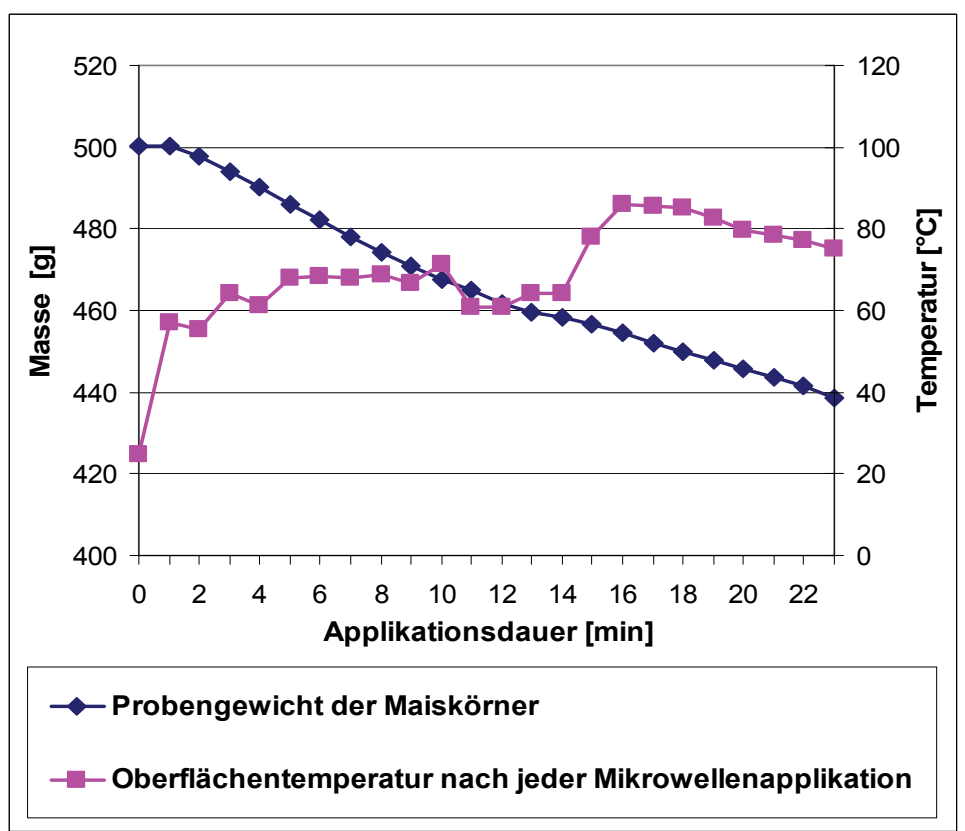

Bild 1.5: Trocknungsverlauf und Oberflächentemperaturänderung von Körnermais im Mikrowellengerät, Anfangsfeuchte: 25,0 \%, Endfeuchte 14,0 \%

Die je Minute entzogene Wassermasse ist in dem folgenden Diagramm (Bild 1.6) dargestellt. $\mathrm{Ab}$ der 16. Minute wurde in Zwei-Minutenabständen appliziert und gemessen. Nach einer Applikationsdauer von 4 Minuten wird die höchste Wasserabgabe von ca. 4,2 g je Minute erreicht, um dann bis zur 14 Minute bis auf ca. 1,5 g Wasserentzug je Minute abzufallen. Erst durch eine Erhöhung der Applikationsdauer auf 2 Minuten erfolgt ab der 16. Trocknungsminute ein erneuter Anstieg der Wasserabgabe auf maximal 2,5 g je Minute nach insgesamt 19 Minuten Trocknungsdauer.

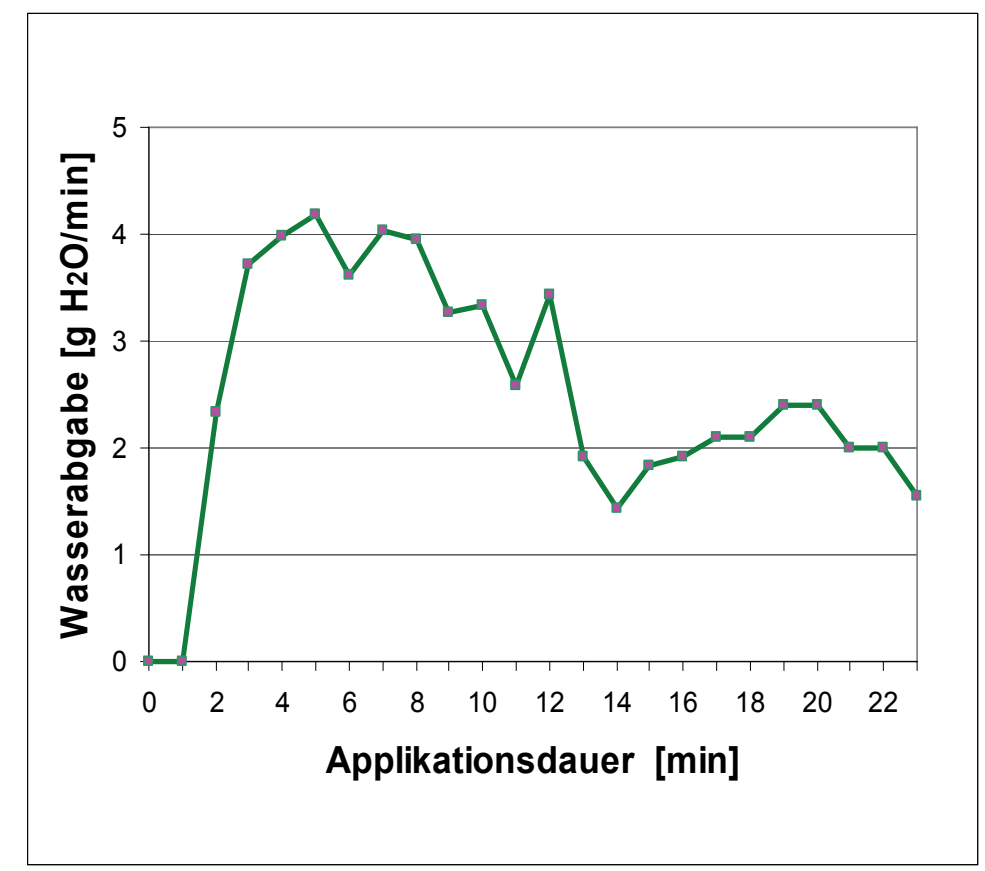

Bild 1.6: Wasserabgabe von Körnermais im Mikrowellengerät, Anfangsfeuchte: 25,0 \%, Endfeuchte $14,0 \%$ 


\subsubsection{Ermittlung der Eindringtiefenverteilung}

Das Ergebnis des Versuches zur Ermittlung der Eindringtiefenverteilung in eine feuchte Körnermaisschicht, ist in Bild 1.7 in dreidimensionaler Form dargestellt.

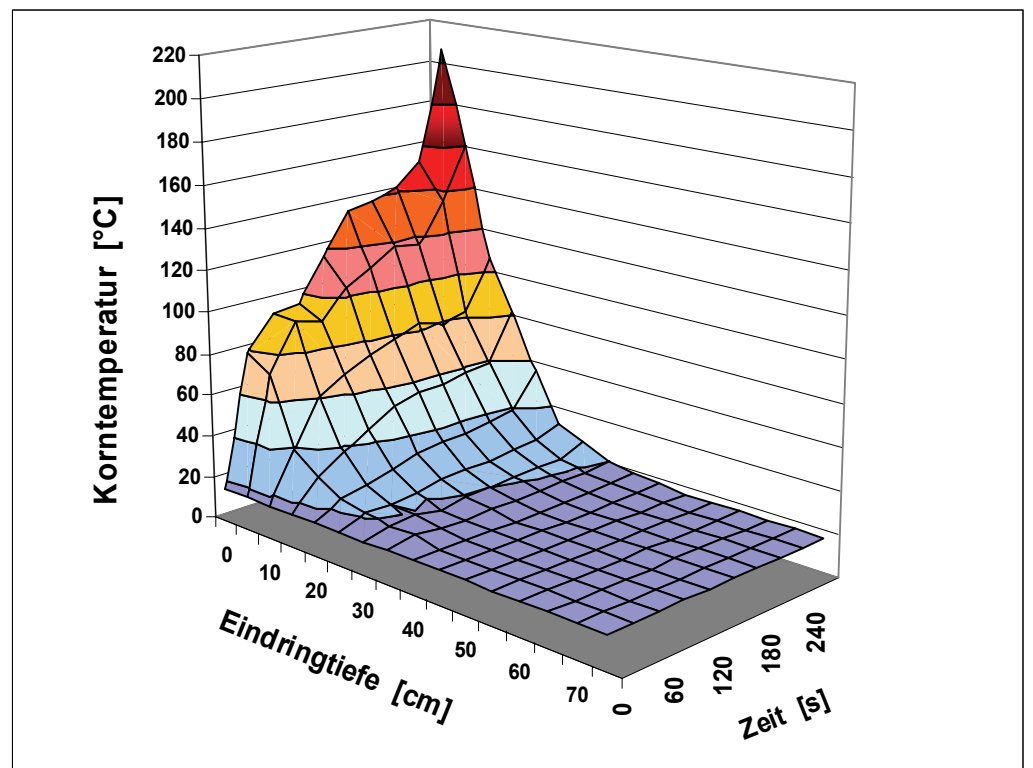

Bild 1.7: Korntemperatur in Abhängigkeit der vertikalen Position im Hohlleiter und der Applikationsdauer von Mikrowellenenergie (600 W), Anfangsfeuchte: 25,0 \%

Das Diagramm 1.7 verdeutlicht den Korntemperaturverlauf als Funktion der Eindringtiefe und Applikationsdauer. An der Körnermaisoberfläche im Hohlleiter liegt die höchste Korntemperatur von $206{ }^{\circ} \mathrm{C}$ nach einer Applikationsdauer von $270 \mathrm{~s}$ vor. Im Abstand von 40 cm unter der Körnermaisoberfläche ist praktisch keine Erwärmung mehr festzustellen. Nach einem steilen Temperaturanstieg zu Beginn der Trocknung am Hohlleiteranfang schwächt sich der Temperaturanstieg dort zunächst etwas ab, gegen Ende der Mikrowellenapplikation steigt die Temperatur jedoch sehr stark an. In Wirkrichtung des mit Körnermais gefüllten Hohlleiters fällt die Temperatur gleichmäßiger $a b$. Der gemessene Temperaturanstieg der Körnermaisschüttung am Hohlleiteranfang war im Vergleich $\mathrm{zu}$ den tiefer im Hohlleiter befindlichen Messorten sprunghafter. Eine zu hohe Energiedichte führte bei diesem Versuch durch eine übermäßige lokale Erwärmung zu starken Bräunungen der Maiskörner, wobei sich mitunter einzelne Körnerverklumpungen ausbildeten, deren Körner dann braun-schwarz wurden. Die Feuchtigkeitsverteilung innerhalb der Kornschüttung war ungleich. Die Kornfarbe ist ein äußerliches Kennzeichnen für eine Qualitätsbeurteilung (Mühlbauer 1980). Farbliche Veränderungen weisen auf Kornschäden hin. In Bild 1.8 ist der Temperaturverlauf nach der Einwirkdauer von 270 Sekunden (entnommen aus Bild 1.7) aufgetragen. Zum Vergleich wurde in das gleiche Diagramm die Exponentialfunktion $\mathrm{y}=\mathrm{e}^{-\mathrm{x}}$ eingetragen, die dem mathematisch idealen Verlauf des Eindringverhaltens entspricht. Der gemessene Temperaturverlauf nach Bild 
1.8 folgt näherungsweise dem Verlauf dieser Exponentialfunktion, wobei bei einer vertikalen Position im Hohlleiter die tatsächliche Korntemperatur meist unter der idealen Annahme liegt. Dieses erklärt sich durch einen Wärmeverlust der Maiskörner durch Wärmeübertragung auf den Hohlleiter. Hierdurch kühlten die Maiskörner im Hohlleiterinnern geringfügig ab und verfälschten somit das Messergebnis geringfügig.

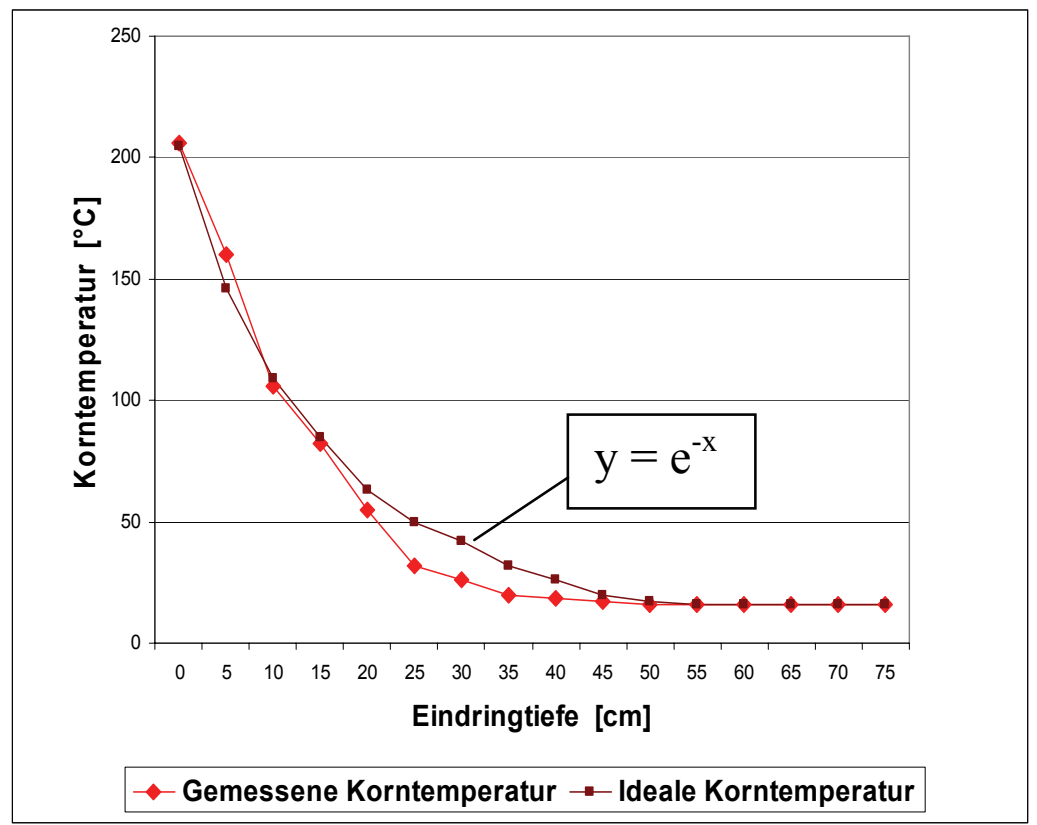

Bild 1.8: Temperaturverlauf als Funktion der vertikalen Position im Hohlleiter

\subsection{Diskussion}

Die Ergebnisse der Versuche zeigen, dass sich Körnermais mit einer Anfangsfeuchte von 25 \% gut zum Erwärmen mit Mikrowellenenergie eignet. Dieses erklärt sich in erster Linie aus dem hohen Wassergehalt und den damit einhergehenden dielektrischen Eigenschaften, die eine zügige und unmittelbare Energieumsetzung in Wärme ermöglichen. Eine Trocknung erfordert dann zusätzlich eine geeignete Feuchteabfuhr. Die Trocknung ist ein kombinierter Vorgang aus Stoff- und Wärmetransport, welcher auch hinsichtlich der mathematischen Beschreibung komplex ist (Lücke 2006). Beim Wassertransport wirken Größen wie Dampfdruck, Temperaturdifferenz, Porengröße und Kapillarlänge. Die verschiedenen Energiearten haben eigene Gesetzmäßigkeiten - auch in Bezug auf das Produkt (Kialbekov et al. 2006). Der Zusammenhang über die Korngeometrie des Einzelkornes und der Schüttung, die Gestaltung und die Befüllung des Arbeitsraumes ist schwer zu formulieren. Zwischen dem Wärme- und Feuchtetransport bestehen Wechselbeziehungen, die sich nur allgemein beschreiben lassen. Für die praktische Anwendung werden neben grundsätzlichen Betrachtungen Messungen bevorzugt 
und ein Vergleich zwischen Berechnungen und Messungen gemacht, wobei die in den Gleichungen 1-7 angegebenen Stoffeigenschaften als konstant angesehen werden. Die Ermittlung der Eindringtiefe von Mikrowelle nach Gleichung 4 mit den von Trabelsi \& Nelson (2003) angegebenen dielektrischen Werten ergibt für Körnermais mit einem Feuchtegehalt von 20,4 \% eine Eindringtiefe von 3,9 cm. Dieser Wert zeigt zu der Eindringtiefenverteilung aus Versuch 1.2 (Bild 1.8) erhebliche Abweichungen. Ein höherer Feuchtegrad als 20 \% müsste eine geringere Eindringtiefe erwarten lassen (Lücke 1992). Durch Messungen und Versuche sind umfassendere Aussagen über die Mikrowelleneigenschaften möglich, weil die produktspezifischen Eigenschaften berücksichtigt werden.

Der Versuch zur Mikrowellentrocknung zeigt, dass Mikrowellenenergie als Trocknungsenergie bei der Trocknung von Körnermais eine sehr kurze Trocknungszeit ermöglicht. Unter Berücksichtigung eines Applikationszyklus von jeweils zwei Minuten und 23 Minuten aktiver Mikrowellentrocknung ergab sich eine Trocknungsdauer der Probe von insgesamt 46 Minuten mit einem Anfangsfeuchtegehalt von $25 \%$ auf einen Endfeuchtegehalt von $14 \%$. Praxistypische Körnermaistrockner erfordern bei reiner Warmlufttrocknung in der Regel eine Trocknungsdauer von etwa drei Stunden, um einen Feuchteentzug von $25 \%$ auf $14 \%$ zu erreichen. Die Probenbereitstellung von Körnermais in der mikrowellendurchlässigen Glasschale erfolgte drei- bis vierlagig. Die Schütthöhe betrug ca. 1,2 bis $2 \mathrm{~cm}$. Durch den konstruktiv im Arbeitsraum befindlichen Feldrührer (Bild 1.3) wurde eine gleichmäßige Produkterwärmung gefördert. Hot spots im Trocknungsgut dürften dadurch unterbunden worden sein. Die Differenz zwischen Minimal- und Maximaltemperaturen, welche der Mittelwertbildung der Oberflächentemperaturen zu Grunde lagen, betrug bis $\mathrm{zu} 3{ }^{\circ} \mathrm{C}$, die Kornfärbung war korntypisch homogen. Nachteilig ist bei der Versuchsdurchführung aber der Umstand, dass während der Trocknung zusätzlich keine Luft durch die Kornschüttung geführt werden konnte, um so die Feuchteabfuhr von der Kornoberfläche zu erhöhen. Ersatzweise wurde die Feuchteabgabe während der Zwischenapplikationszeit durch Rühren der Probe außerhalb des Arbeitsraumes unterstützt. Daher ist zu erwarten, dass die Trocknung von Körnermais durch den Einsatz von Mikrowellen weiter begünstigt werden kann, wenn während der Applikation Luft zur Abfuhr der an die Kornoberfläche diffundierten Feuchtigkeit das Trocknungsgut durchströmt. Weiterhin sollte während der Trocknung das Trocknungsgut bewegt werden können, damit die Mikrowellen möglichst gleichmäßig die Produkterwärmung unterstützen. Hierbei ist davon auszugehen, dass eine passive Bewegung des Trocknungsgutes, z. B. auf einem Drehteller oder einem Förderband, in einem Mikrowellentrockner nicht so vorteilhaft wirken kann, als wenn das Trocknungsgut während der Mikrowellenapplikation 
selbst durchmischt wird. Die Produktbewegung muss so intensiv ausgeführt sein, dass eine optimale Trocknung ermöglicht wird, ohne dass sich hohe lokale Korntemperaturen (hot spots) ausbilden können. Aus den Ergebnissen von Gunasekaran (1990) zeigt sich, dass ein über die Trocknungsdauer wirkendes Impuls-Pausenverhältnis die Trocknungsdauer und den erforderlichen Energieaufwand beeinflusst. Applikationsdauer und Energiedichte der Mikrowellenapplikation müssen auf das Trocknungsgut im Arbeitsraum gleichmäßig wirken. Dieses ist bei weiterführenden Versuchen zu berücksichtigen.

In Bild 1.5 ist der Wasserverlust über der Applikationsdauer bei der Mikrowellentrocknung mit Körnermais dargestellt. Aufgrund des relativ geringen Anfangsfeuchtegehaltes von $25 \%$ und fehlender Trocknungsluft zur Abfuhr der Oberflächenfeuchte stellte sich in Versuch 1.1 keine Beharrungstemperatur im Trocknungsgut ein, wie dieses von Krischer \& Kast (1978) für die beginnende Trocknung im ersten Trocknungsabschnitt bei einem hohen Flüssigkeitsgehalt des Gutes beschrieben ist. Der Linienverlauf entsprach prinzipiell dem des zweiten oder dritten Trocknungsabschnittes, wobei nach Mühlbauer (1974) der dritte Trocknungsabschnitt bei der Körnermaistrocknung erst bei einem Feuchtegehalt unter $9 \% \mathrm{zu}$ beobachten ist. Dieser Feuchtebereich ist aber bei der Maistrocknung von geringer Bedeutung, da er weit unter dem lagernotwendigen Zustand liegt. Daher wurde der Versuch 1.1 auch bei einem Endfeuchtegehalt von $14 \%$ beendet. Nach Abkühlen der Probe an der Luft verringerte sich der Feuchtegehalt auf $13,5 \%$.

Für weiterführende Anwendungen eines Mikrowelleneinsatzes zur Körnermaistrocknung lässt sich aus Bild 1.7 erkennen, wie sich die zeitliche und räumliche Eindringtiefenverteilung in eine feuchte Körnermaisschüttung ausbilden. Als Ursachen für die teilweise sprunghaften Temperaturänderungen werden die sich zwar ähnliche, mitunter aber dennoch leicht abweichende Position des jeweiligen Messortes des Einstechfühlers in Verbindung mit möglichen geringfügigen Abweichungen hinsichtlich der Messintervalle vermutet. Auch können Einflüsse der Mikrowellenausbreitung im Hohleiter zur Entstehung von hot spots nicht ausgeschlossen werden. Durch weiterführende Untersuchungen ist noch zu klären, ob oder durch welche Applikationsbedingungen die durch Mikrowellen bewirkte Erwärmung des Einzelkorns nicht hinreichend homogen ist, bzw. wie eine ungleiche Erwärmung des Einzelkornes in einer Schüttung nachhaltig verhindert werden kann. Aus Bild 1.8 geht hervor, dass der Versuchsaufbau und die -durchführung sowie die gewählten Messintervalle und Messorte im Sinne der Temperaturmessung günstig gewählt wurden. 
Bei der Warmlufttrocknung beeinflussen die Strömungszustände der Trocknungsluft den Trocknungsverlauf. In der Praxis haben sich je nach Trocknerbauart Trocknungsschichtdicken zwischen 15 und $150 \mathrm{~cm}$ (Toftdahl) bewährt. Aus den Ergebnissen des Versuches 1.2 leitet sich $\mathrm{ab}$, dass beim Einsatz von Mikrowellen mit einer Frequenz von 2,45 GHz Schichtdicken zwischen 4 bis $20 \mathrm{~cm}$ zur Erwärmung zweckmäßig sind. Hierbei ist zu beachten, dass sich die dielektrische Erwärmung sehr viel schneller als die konvektive Erwärmung vollzieht und eine geringere Schichtdicke nicht zur Reduktion der Trocknereffizienz führt.

\subsection{Schlussfolgerungen}

Bei der Trocknung von Körnermais wird in der Praxis überwiegend die Warmlufttrocknung eingesetzt. Warmluft dient zunächst zum Austreiben der Feuchtigkeit aus dem Korn und zudem der Feuchtigkeitsabfuhr. Trocknungsverfahren sind heute weitestgehend optimiert, so dass eine Steigerung der Trocknungsleistung - also Verkürzung der Trocknungsdauer und Verringerung der Trocknungsenergie - bei Erhalt der Qualität technisch kaum noch möglich ist. Bei Verwendung der Mikrowelleenergie wird die Feuchtigkeit aus dem Korn ausgetrieben, wobei die Feuchtigkeitsabfuhr Warmluft erfordert. Durch Verwendung von Mikrowellen lassen sich die erforderlichen Trocknungszeiten deutlich verkürzen. Es stellt sich daher die Frage, inwieweit eine Verfahrenskombination aus Warmlufttrocknung und Mikrowelleneinsatz dazu beitragen kann, bei der Körnermaistrocknung den erforderlichen Energieeinsatz zu reduzieren und die erforderliche Trocknungsdauer zu verringern. Hierbei ist vor allem zu untersuchen, ob zur Unterstützung der Warmlufttrocknung eine Mikrowellenapplikation zeitgleich oder nacheinander erfolgen muss. Dieses ist ein maßgebliches Kriterium für die konstruktive und verfahrenstechnische Trocknerdimensionierung.

Vergleicht man beide Versuche miteinander, so beobachtet man bei Versuch 1.1 keine deutlich unterschiedliche Erwärmung der Körnermaisprobe im Arbeitsraum des LaborMikrowellengerätes. Bei Versuch 1.2 weist die im Hohlleiter befindliche Körnermaisprobe bereits nach kurzer Applikationsdauer eine unterschiedliche Temperaturverteilung auf. Bei dieser Versuchsanordnung war keine Möglichkeit vorhanden, um die Mikrowellen gleichmäßig auf die gesamte Körnermaisprobe wirken zu lassen. Aus Bild 1.7 zeigt sich daher, dass sich mit zunehmender Eindringtiefe sowohl die Produkttemperatur als auch die Temperaturunterschiede stark abschwächen. Daraus leitet sich ab, dass es zur vorteilhaften Anwendung von Mirkowellenenergie zur Maistrocknung erforderlich ist, entweder der Mikrowellenenergie auf das Produkt gleichmäßig im gesamten Trocknungsraum wirksam werden zu lassen, oder das 
Produkt selbst im Arbeitsraum der Mikrowelle so $\mathrm{zu}$ bewegen, dass eine gleichmäßige Umsetzung der Mikrowellenenergie in Produkterwärmung erreicht wird. Zur Optimierung des Trocknungsverlaufes sind praxistypische Körnermais-Warmlufttrockner nicht als Ruheschichttrockner ausgebildet. In weiteren Versuchen ist zu klären, ob die Mechanismen zur Kornbewegung bewährter Körnermais-Warmlufttrocknungssysteme für eine auf Mikrowelleneinsatz basierende Trocknung oder unterstützende Trocknung übertragbar sind.

Ein direkter Vergleich bzw. eine direkte Umsetzbarkeit der im Labormaßstab gewonnenen Erkenntnisse auf größere Anlagen im Technikumsmaßstab ist aber nur bedingt möglich. Sie geben jedoch wertvolle Ansätze für eine Auslegung von Trocknungsanlagen, die mit Mikrowellenenergie bzw. mit Warmluft und Mikrowellenenergie betrieben werden. Es ist deshalb sinnvoll, Versuche mit unterschiedlichen Energiekombinationen durchzuführen und die Ergebnisse stichprobenartig mit theoretischen Ansätzen $\mathrm{zu}$ vergleichen und $\mathrm{zu}$ interpretieren. Um Wechselwirkungen innerhalb des Gesamtsystems „kombinierte Trocknungsanlage“ beschreibbarer und damit für größere Anlagen anwendbar zu machen, sind weitere Versuche im Technikumsmaßstab und größer notwendig. 


\section{Teil}

\section{Der Einsatz von Mikrowellen zur Maistrocknung - Anwendungen im Technikumsmaßstab}

\section{Zusammenfassung}

Konventionelle Warmlufttrocknungssysteme für die Körnermaistrocknung sind technisch weitgehend ausgereift, wobei aus der Praxis möglichst hohe Trocknungsleistungen bei geringen Trocknungskosten gefordert werden. Im Hinblick darauf wurde am Department für Agrartechnik der Universität Göttingen und im Labor für Mikrowellentechnik am Institut für Technologie- und Wissenstransfer, TWS, im Kreis Soest e.V. untersucht, wie sich durch eine Verfahrenskombination aus Warmlufttrocknung und Mikrowellenapplikation Prozesse der Körnermaistrocknung optimieren lassen. Hierzu wurden Versuche im Technikumsmaßstab durchgeführt. Die Ergebnisse zeigen auf, unter welchen Bedingungen eine Kombinationstrocknung verfahrenstechnisch sinnvoll ist. 


\subsection{Einleitung}

Die rechtzeitige und schonende Konservierung von Körnermais und anderen landwirtschaftlichen Körnerfrüchten stellt angesichts weltweit hoher Verluste an Nahrungsmitteln im Nacherntebereich eine bedeutende Aufgabe für die agrartechnische Forschung dar. Vor dem Hintergrund, dass landwirtschaftlich erzeugte Rohstoffe zunehmend als Basis verwertbarer Grundwerkstoffe in der Industrie, Energie- oder Medizintechnik eingesetzt werden, gewinnt der Einsatz an die spätere Verwendung angepasster Trocknungsverfahren zunehmend an Bedeutung (Mellmann et al. 2005, Münzing 1995).

Im Gegensatz zur reinen Warmlufttrocknung besteht durch die Applikation von Mikrowellen eine grundsätzlich andere Möglichkeit den Energieeintrag in das Trocknungsgut durchzuführen. Hierbei wird die physikalische Eigenschaft genutzt, dass die Energie direkt in den Wassermolekülen in Wärme umgesetzt wird. Die Mikrowellenenergie wirkt im Innern des Trocknungsgutes, ohne dass diesem Effekt eine auch hierauf beruhende Wärmeübertragung von der Kornschale zum Korninnern zwingend vorausgeht. Hierdurch wird in aller Regel der Entfeuchtungsvorgang erheblich beschleunigt (Lücke 2006).

\subsection{Stand der Technik}

Die derzeit marktgängigen Trocknungssysteme stellen in Bezug auf die Wasserabgabefähigkeit des Körnermais dem Produkt stets ein überhöhtes Energieangebot zur Verfügung, um den erforderlichen Dampfdruck zum Austritt des Wassers aufzubauen. Der notwendige Energieeintrag in das Produkt wird über dieses Kriterium bestimmt (Metaxas 1983). Ziel ist es, möglichst kurze Trocknungszeiten zu erreichen. Feuchtes Getreide oder auch feuchter Mais reagieren empfindlicher auf hohe Korntemperaturen als trockene Ware (Beke 1997, Mühlbauer 1974). Die Gleichmäßigkeit der Korntemperatur in zu trocknenden Produktschüttungen ist für eine homogene und möglichst zügige Trocknung wichtig. Je höher der Energieeintrag in das Trocknungsgut ist, desto größer muss die Kornbewegung der Maisschüttung sein, um der Gefahr lokal hoher Korntemperaturen entgegenzuwirken (Krischer und Kast 1978).

Während der Trocknung innerhalb des ersten Trocknungsabschnittes sind bei feuchtem Körnermais hohe Warmlufttemperaturen möglich. Sobald der Wärmeeintrag und der daraus folgende Feuchtigkeitsaustrag aus dem Korn nicht mehr gleichgewichtig erfolgt, können 
Überhitzung und Kornschäden auftreten. Dieser Effekt lässt sich nur dadurch mindern, indem die Warmlufttemperatur verringert bzw. an die jeweiligen Trocknungsverhältnisse angepasst wird. (Maltry et al. 1975, Skriegan 2005, Toftdahl 1987).

Durch Wärmeleitung wird die zur Trocknung notwendige Energie von der Produktoberfläche ins Innere geleitet, die Wärmeleitfähigkeit des Stoffes ist von seinen thermischen Eigenschaften und seinem Feuchtegehalt abhängig. Die Wärmeleitfähigkeit verschlechtert sich mit geringer werdender Gutfeuchtigkeit. Durch Feuchteleitung erfolgt der Transport der Flüssigkeit aus dem Innern des Trocknungsgutes zur Oberfläche. Die Feuchteleitfähigkeit hängt von der Struktur der Feststoffmatrix ab und ist damit gutcharakteristisch. Die Transporteigenschaften für die Feuchte werden durch den Kapillardruck bestimmt, der mit abnehmender Porengröße und mit abnehmendem Feuchtegehalt ansteigt (Gröber et al. 1963). Stofftransport in der gasförmigen Phase findet infolge von Dampfdiffusion statt, entscheidend für dessen Größe ist der Diffusionskoeffizient des Dampfes im Gas bzw. der Trocknungsluft und der Diffusionswiderstand des Feststoffes. Dieser Diffusionswiderstand ist vom Feuchtegehalt abhängig und nimmt mit abnehmendem Feuchtegehalt zu. Hierdurch können Diffusionsbarrieren an der Gutoberfläche entstehen, die die Trocknungszeit verlängern können (Schlünder 1998).

Bei der Mikrowellentrocknung erfolgt im Gegensatz zur Warmlufttrocknung eine Verdampfung der Feuchtigkeit im Korninnern (Askeland 1996, Heindl 1993, Hippel von 1954). Die äußeren Schichten bzw. Schalen des Maiskorns bleiben bei einer Mikrowellenapplikation bis zum Ende des Trocknungsprozesses diffusionsfähiger, weil der Feuchtetransport vom Korninnern an die Oberfläche nachgeführt wird (Böckelmann et al. 2007). Bei der Warmlufttrocknung erfolgt der Energieübergang stets von der Kornoberfläche aus, so dass diese Bereiche auch zuerst getrocknet werden. Die Wärme- und Feuchtedurchlässigkeit verringern sich. Zudem wird Warmluft benötigt, um das an die Kornoberfläche gelangte Wasser abzuführen (Kröll und Kast 1989).

In seinen Versuchen zur Mikrowellenapplikation an Möhrenscheiben hat Tarsa (1991) festgestellt, dass es aus ökonomischen Gründen angebracht ist, das leicht entfernbare Wasser durch Konvektionstrocknung zu entziehen, und erst später mit der Mikrowellenapplikation zu beginnen. Aufgrund der niedrigen Wassergehalte zum Trocknungsende wird die Mikrowellenenergie schlecht in Wärme umgewandelt. Die Endtrocknung ist daher vorteilhaft mit Konvektionsenergie abzuschließen, wobei kurzzeitige Mikrowellenimpulse die 
Trocknungsgeschwindigkeit erhöhen. Im Gegensatz zu einer unabhängig von der Schnittebene weitgehend offenporigen Struktur von Möhrenscheiben zeigen Maiskörner aufgrund ihres Aufbaues ein grundlegend anderes Wasserabgabeverhalten. Beim Maiskorn liegt das Wasser nicht nur in reiner Form, sondern auch als Nährlösung vor. Daher ist zur Entfeuchtung aus dem Maiskorn zusätzlich zur Verdampfungswärme die Bindungswärme zuzuführen, die von der Feuchtebindung abhängig ist. Beim Maiskorn liegt physikalisch-chemisch gebundene Feuchtigkeit und physikalisch-mechanisch gebundene Feuchtigkeit vor (Krischer 1992, Mühlbauer 1974). Sowohl aus den theoretischen Betrachtungen als auch an Trocknungsversuchen mit Kartoffelscheiben hat Lücke (1992) festgestellt, dass ergänzend zur Warmlufttrocknung eine Mikrowellenapplikation dann vorteilhaft wirkt, wenn die Einkopplung der Mikrowellenenergie sowohl konstruktiv technisch als auch im Sinne des Trocknungsverlaufes aufeinander abgestimmt ist. Es stellt sich daher die Frage, welche verfahrenstechnischen und konstruktiven Eigenschaften eines Trockners erforderlich sind, damit mit Mikrowellenenergie die Warmlufttrocknung vorteilhaft unterstützt werden kann.

\subsection{Material und Methoden}

\subsubsection{Aufbau der Versuchsanlage}

Der metallische Trockner besteht aus einem stehenden zylindrischen Mischer mit einem ebenen Lochboden (1,5 mm Lochdurchmesser), worunter sich der Abluftraum befindet (Bild 2.1). Der Trocknungsraum hat einen Durchmesser von 0,45 m und eine Höhe von 0,50 m. Das entspricht einem Volumen von 0,08 $\mathrm{m}^{3}$. Im Gegensatz zu bekannten Behältertrocknungen wird die angewärmte Prozessluft oberhalb der Gutschicht durch eine seitliche Zuluftöffnung eingesaugt und die Abluft unterhalb des Lochbodens von einem Radialgebläse abgeführt (Bild 2.2). Durch die saugende Betriebsweise bleibt die Anlagenperipherie staubfrei und trocken, hingegen werden Abluftfeuchte, Abrieb oder Maisstaub direkt abgeführt (Ciesler 1986). In die Behälterwandung ist im Bereich des Zuluftanschlusses ein Edelstahlgitter $(1,5 \mathrm{~mm}$ Lochdurchmesser) eingearbeitet, so dass die Warmluft nahezu strähnenfrei in den Trocknungsraum einströmt (Matthis 1956). Gleichzeitig hat das Edelstahlgitter die Aufgabe, den Austritt von Mikrowellen nach außen zu verhindern. Im Ergebnis ist ein absätzig arbeitender, so genannter Chargenmischertrockner konzipiert worden. Auf diese Weise ist es möglich, alle während der Trocknung relevanten Prozesszustände separat einzustellen und zu bilanzieren. 


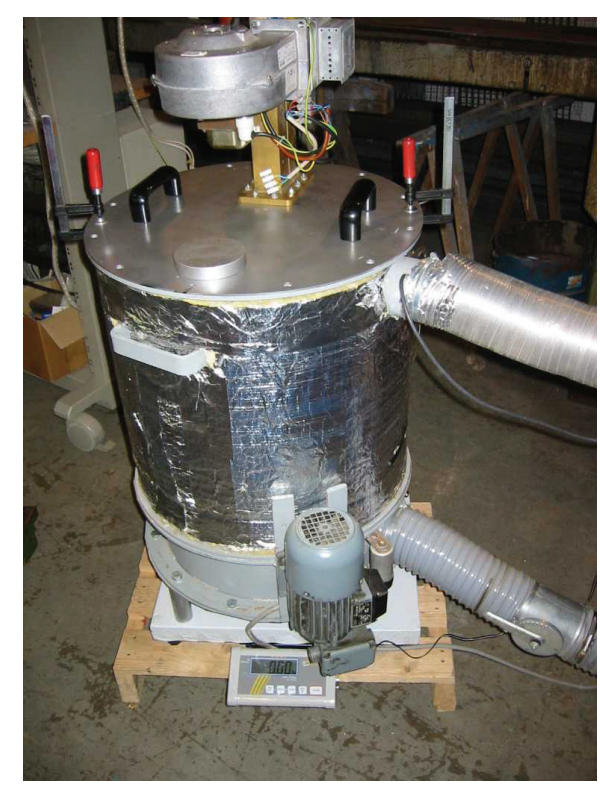

Bild 2.1: Chargenmischertrockner

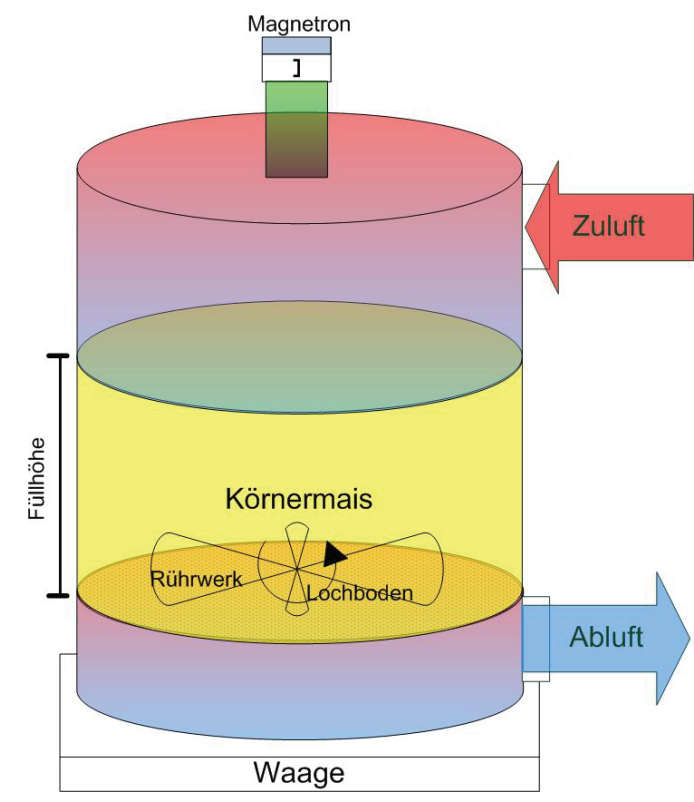

Bild 2.2: Aufbau der Versuchsanlage

Der Chargenmischertrockner verfügt über zwei austauschbare Abdeckungen. Mit Hilfe eines hitzebeständigen Glasdeckels lässt sich der Trockner als reiner Warmlufttrockner betreiben, wobei sich während des Trocknungsvorganges die Veränderungen von Volumen, Farbe und Fließverhalten des Trocknungsgutes gut beobachten lassen. Der zweite Deckel besteht aus einer Metallscheibe, auf dem exzentrisch der Hohlleiter mit einem aufgesetzten Industriemagnetron mit $1200 \mathrm{~W}$ Sendeleistung bei einer Frequenz von 2,45 GHz positioniert ist. Die Sendeleistung ist zwischen 120 und $1200 \mathrm{~W}$ Hochfrequenz stufenlos regelbar. Das verwendete Magnetron hat einen Wirkungsgrad von ca. $70 \%$, daraus ergibt sich eine elektrische Aufnahmeleistung von 1800 W. Die Abwärme des Magnetrons wird bei diesem Trockner technisch nicht weiter verwertet. Im industriellen Mikrowelleneinsatz lassen sich in der Regel ca. 50 \% der Abwärme verfahrenstechnisch sinnvoll nutzen (Püschner 1964). Durch ein frequenzgesteuertes Radialgebläse lässt sich ein Prozessluftstrom zwischen 30 bis $350 \mathrm{~m}^{3}$ pro Stunde einstellen. Das entspricht einer spezifischen Trockenluftrate von 380 bis $4800 \mathrm{~m}^{3}$ Prozessluft je Kubikmeter Trockengut und Stunde (Kröll und Kast 1989, Maltry et al. 1975, Zscheischler et al. 1990). Die Trocknungsluft wird über zwei in Reihe angeordnete elektrisch betriebene Widerstandsheizregister mit je $1700 \mathrm{~W}$ erwärmt, wobei die Warmlufttemperatur von einem PID-Regler stufenlos zwischen der Umgebungstemperatur und $200{ }^{\circ} \mathrm{C}$ einstellbar ist. Für die Energiebilanzierung der Trocknungsversuche sind die elektrische Energieaufnahme der Warmlufterzeugung und der dielektrischen Erwärmung durch getrennte Energiezähler erfasst worden. Während der Trocknung wird das Trocknungsgut durch eine motorisch angetriebene Rührwelle mit 10 Umdrehungen pro Minute von zwei Rührarmen durchmischt. Dadurch ist 
eine homogene Produktverteilung während der Trocknung gegeben. Die Einschaltzeiten des Rührwerkes können mit einem Arbeitszeit-Pausenrelais stufenlos verstellt werden. Im Betriebszustand ist der Trockner auf einer digitalen Waage mit einer Auflösung von 0,050 kg positioniert, so dass die Gewichtsänderung des Trocknungsgutes durch die Wasserabgabe kontinuierlich aufgezeichnet werden kann.

\subsubsection{Versuchsdurchführung}

Bei den Versuchen zur Körnermaistrocknung hat sich bei dieser Anlage eine Chargengröße zwischen 10 und $30 \mathrm{~kg}$ bewährt. Dieses entspricht einer prozentualen Trocknerraumausnutzung von ca. 18 bis 55 \%. Durch eine in Abhängigkeit der Befüllhöhe einstellbare Durchströmung der Schüttung lassen sich unterschiedliche Strömungsverhältnisse erfassen (Martinez 2001). Die Trocknerkonstruktion ist so ausgeführt, dass die Wirkrichtung der zuschaltbaren Mikrowelleapplikation mit dem Luftstrom gleichgerichtet ist. Zur Energie- und Leistungsbilanzierung werden während des Trocknerbetriebes folgende Messdaten erfasst:

- Systemgewicht (Probengewicht nach Tara-Abzug)

- Temperatur, Feuchtegehalt und Strömungsgeschwindigkeit der Zu- und Abluft

- Trocknungsguttemperatur (nur bei Warmlufttrocknung möglich)

- Druckdifferenz

- Warmluft- bzw. Heizleistung

- Mikrowellenenergie bzw. Mikrowellenleistung

- Leistungsbedarf zur Prozessluftförderung

- Einschaltdauer Rührwerk

Die Feuchtegehaltsbestimmung des eingesetzten Körnermais sind mit einem geeichten Getreidefeuchtemessgerät (Pfeuffer HE 90) sowie einem thermogravimetrischen Messgerät (Ohaus MB 45) durchgeführt worden. Referenzmessungen sind im Trockenschrank abgeglichen worden. Für die durchgeführten Trocknungsversuche ist erntefrischer Körnermais verwendet worden. Im Rahmen dieser Ausarbeitung werden drei Versuche am Chargenmischertrockner beschrieben, wobei der erste Versuch aus zwei Teilversuchen besteht: 
Versuch 2.1: Ermittlung des Eindringverhaltens in eine Körnermaisschüttung nur mit Mikrowellenerwärmung

2.1a: ohne Durchmischen mit dem Rührwerk

2.1b: mit anschließendem Durchmischen mit dem Rührwerk

Versuch 2.2: Ermittlung des Trocknungsverhaltens bei alleiniger Warmlufttrocknung

Versuch 2.3: Ermittlung des Trocknungsverhaltens bei kombinierter WarmluftMikrowellentrocknung

\subsubsection{Material}

Für die Versuche ist Körnermais der Sorte Boss verwendet worden, der einen Tag vor der Versuchsdurchführung geerntet worden ist. Die Abreifebedingungen waren an dem Standort aufgrund einer sandigen Bodenbeschaffenheit nicht optimal. Im stehenden Bestand waren Anzeichen von Trockenstress erkennbar. Die Kolbengröße war teilweise heterogen, ebenso die Größe der Körner selbst. Der Erntefeuchtegehalt betrug $29 \%$.

\subsubsection{Versuch 2.1: Ermittlung des Eindringverhaltens in eine} Körnermaisschicht nur mit Mikrowellenerwärmung

In Vorversuchen hat sich gezeigt, dass ein freier Abstand der am Magnetron austretenden Mikrowellen bis zum Auftreffen auf die feuchte Körnermaisoberfläche günstig ist, der der einbis dreifachen Wellenlänge der Mikrowellenfrequenz entspricht. Der Chargenmischertrockner ist mit $25 \mathrm{~kg}$ Körnermais mit einem Feuchtegehalt von 29,0 \% und einer Korntemperatur von ca. $11{ }^{\circ} \mathrm{C}$ befüllt worden. Um Feuchtigkeit abzuführen, ist die Schüttung mit Kaltluft $\left(20,3{ }^{\circ} \mathrm{C}\right.$, $65,8 \%$ rel. Luftfeuchtegehalt) bei einem Volumenstrom von $100 \mathrm{~m}^{3} \mathrm{~h}^{-1}$ durchströmt worden. Hierdurch hat sich eine geringfügige Verzerrung des Temperaturausbreitungsprofils im Mais ergeben. Für die Dauer von $300 \mathrm{~s}$ wurden Mikrowellen mit einer Sendeleistung von $600 \mathrm{~W}$ appliziert. Hiernach ist der Deckel mit dem integrierten Magnetron für die Aufnahmen im sichtbaren Bereich (Bild 2.3) und Infrarotaufnahmen (Bild 2.4 - 2.10) entfernt worden. Im ersten Teil des Versuches (2.1a) ist der Körnermais nicht bewegt worden. Im zweiten Teil des Versuches (2.1b) wurde das Rührwerk zunächst für $1 / 2$ Umdrehung, dann für ein, für zwei, für zehn und weiterhin für 20 Umdrehungen (2 Minuten) eingeschaltet und die Oberfläche der im Trockner befindlichen Körnermaisschüttung mit der Infrarotkamera (FLIR Therma-CAM Typ 
PM 575) aufgenommen. Durch die Abfolge an Fotographien wird die Wirkung der Durchmischung und Temperaturverteilung der Maiskörner sichtbar.

2.3.2.3 Versuch 2.2: Ermittlung des Trocknungsverhaltens bei alleiniger

\section{Warmlufttrocknung}

Der Versuch 2.2 ist - ebenso wie Versuch 2.3 - mit je $25 \mathrm{~kg}$ Feuchtmais durchgeführt worden.

Zunächst wurde mit: $\mathrm{m}_{\mathrm{H} 2 \mathrm{O}}=\mathrm{m}_{\text {Frischmasse }} \cdot \frac{\mathrm{F}_{\mathrm{A}}-\mathrm{F}_{\mathrm{E}}}{100-\mathrm{F}_{\mathrm{E}}}$

$\begin{array}{ll}\mathrm{m} \mathrm{H}_{2} \mathrm{O}=\text { Masse Wasser } & {[\mathrm{kg}]} \\ \mathrm{m}_{\text {Frischmasse }}=\text { Masse Feuchtmais } & {[\mathrm{kg}]} \\ \mathrm{F}_{\mathrm{A}}=\text { Anfangsfeuchtegehalt } & {[\%]} \\ \mathrm{F}_{\mathrm{E}}=\text { Endfeuchtegehalt } & {[\%]}\end{array}$

die Masse Wasser ermittelt, die aus dem Körnermais entfernt werden muss, um das Zielgewicht für einen Endfeuchtegehalt von ca. 14,0 \% zu erreichen. Während des Trocknens sind keine Feuchtegehaltsmessungen am Produkt durchgeführt worden, stattdessen ist der Wasserentzug während des Trocknungsvorganges gravimetrisch mit Hilfe der Waage ermittelt worden. Für die ersten 45 Minuten ist eine Zulufttemperatur von $90{ }^{\circ} \mathrm{C}$ gewählt, anschließend ist die Zulufttemperatur bis zum Ende des Versuchs auf $130{ }^{\circ} \mathrm{C}$ erhöht worden. Der Trockner ist mit konstanter Prozessluftmenge von ca. $200 \mathrm{~m}^{3} \mathrm{~h}^{-1}$ betrieben worden. Das Rührwerk ist permanent in Betrieb gewesen. Die Abluftzustände sind mit einem Klimamessgerät (Testo 400), bestehend aus einem Temperatur-Luftfeuchte-Kombinationsfühler mit angeschlossenem Datenlogger aufgezeichnet worden.

2.3.2.4 Versuch 2.3: Ermittlung des Trocknungsverhaltens bei kombinierter Warmluft-Mikrowellentrocknung

Für den Versuch 2.3 sind die aus Versuch 2.2 gewählten Zuluftbedingungen übernommen worden. Im Unterschied zu Versuch 2.2 ist nach Unterschreiten des Abluftfeuchtegehaltes von $75 \%$ rel. Feuchte Mikrowellenenergie mit einer Sendeleistung von $360 \mathrm{~W}$ für 30 Minuten zugeschaltet worden. Das Rührwerk ist ebenfalls permanent in Betrieb gewesen. 


\subsection{Ergebnisse}

\subsubsection{Versuch 2.1a}

Bild 2.3 zeigt den mit Mais gefüllten Chargenmischertrockner von oben. Bild 2.4 zeigt die Maisoberfläche aus Bild 2.3 als Infrarotbild. Man erkennt eine nahezu gleichmäßige Temperaturverteilung der Oberfläche zwischen 5,6 und 13,6 ${ }^{\circ} \mathrm{C}$. Helle Bereiche bedeuten hohe Temperaturen, dunkle Bereiche stehen für tiefe Temperaturen. An dem Messpunkt Sp1 beträgt die Temperatur $7,0^{\circ} \mathrm{C}$. Die Kamera wurde über dem Chargenmischertrockner fest montiert, die Stelle dieses Messpunktes ist während der Versuche nicht verändert worden.

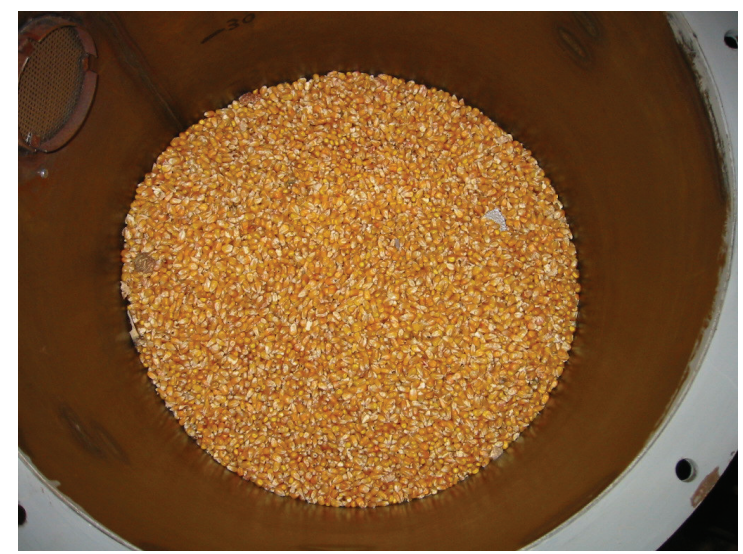

Bild 2.3: Ansicht in den Arbeitsraum des Chargenmischertrockners

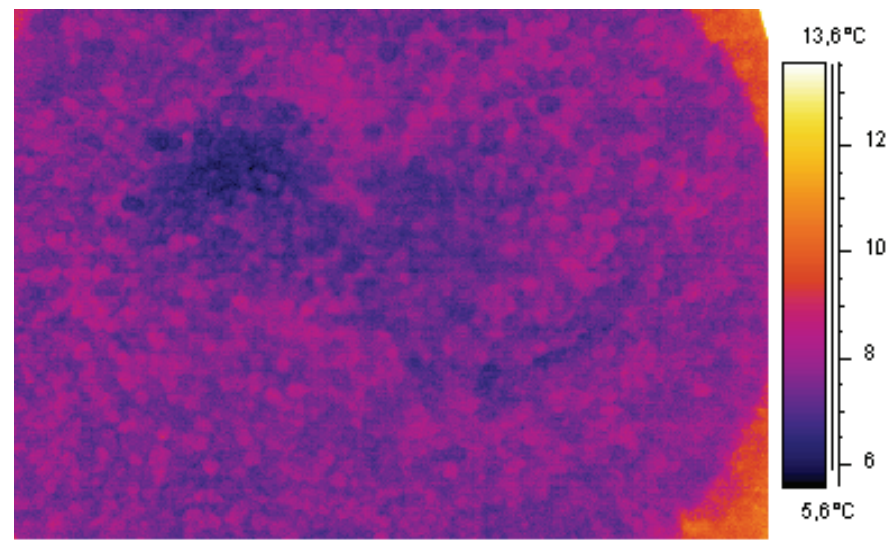

Bild 2.4: Infrarotaufnahme mit erntefrischem Mais Temperatur: $5,6-13,6{ }^{\circ} \mathrm{C}$

Nach der Zufuhr von Mikrowellenenergie hat sich die Oberflächentemperatur unterschiedlich erhöht. Die Temperaturverteilung ist in Bild 2.5 gezeigt. Sie lag zwischen 19,7 und $78,8{ }^{\circ} \mathrm{C}$. An dem Messpunkt Sp1 betrug die Temperatur $23,1{ }^{\circ} \mathrm{C}$. In Bild 5 erkennt man ausgeprägte heiße Stellen in relativ regelmäßigen Abständen voneinander, die sich durch das Wirkfeld der Mikrowellen im Trocknungsraum ergeben.

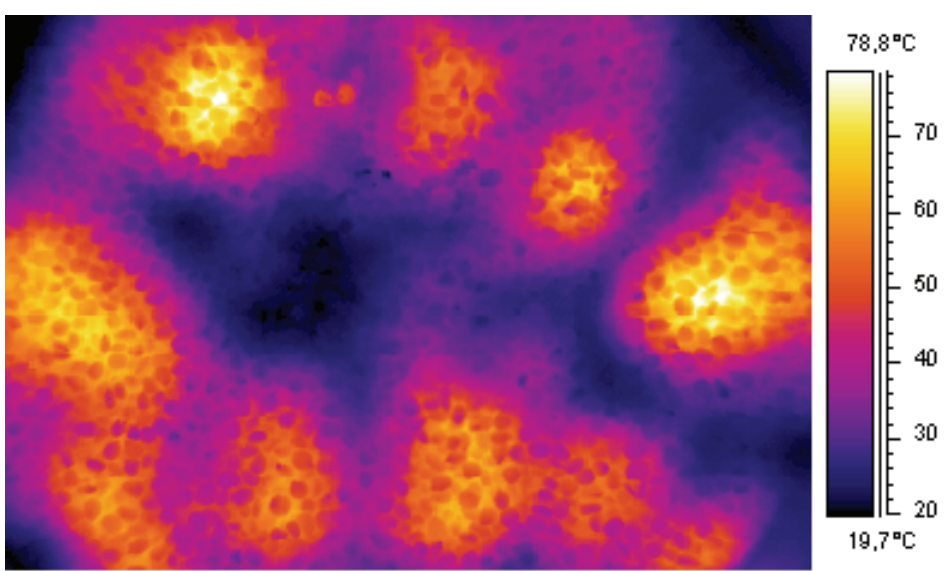

Bild 2.5: Temperaturverteilung des durch Mikrowellen erwärmten Körnermaises.

Temperatur: $19,7-78,8^{\circ} \mathrm{C}$ 


\subsubsection{Versuch 2.1b}

Die Ergebnisse dieses Versuches sind in einer Abfolge von Infrarotbildern wiedergegeben. Sie beginnen mit dem Bild 2.5 von Versuch 2.1a. Mit ihnen soll gezeigt werden wie sich die Temperaturverteilung an der Oberfläche, ausgehend vom erwärmten Zustand (Bild 2.5) ändert, sobald das Rührwerk für eine verschiedene Anzahl Umdrehungen (Bild 2.6 bis 2.10), eingeschaltet wird. Ein Temperaturausgleich wird dadurch gefördert. Man erkennt an der jeweils rechten Temperaturskala, dass die Temperaturspreizung sich erst nach zwei und mehr Umdrehungen verringert (Bild 2.8 bis 2.10).

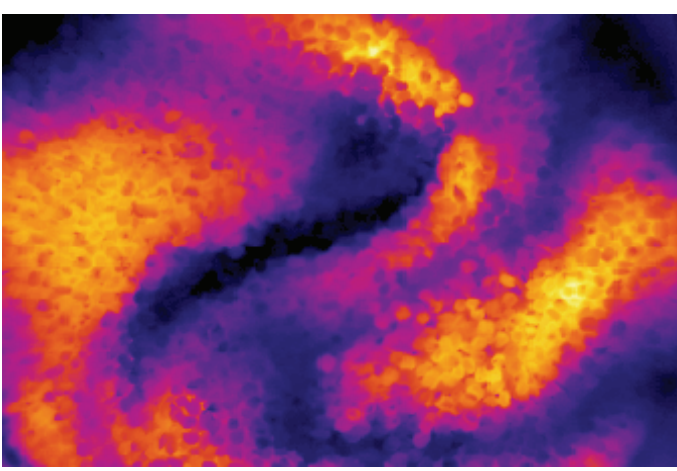

Bild 2.6: Infrarotaufnahme nach $1 / 2$ Umdrehung Temperatur: $19,7-78,8^{\circ} \mathrm{C}$

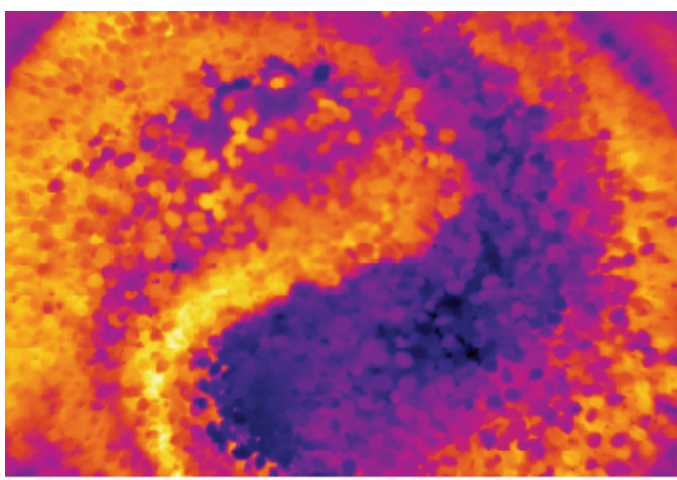

Bild 2.8: Infrarotaufnahme nach 2 Umdrehungen Temperatur: $17,5-55^{\circ} \mathrm{C}$

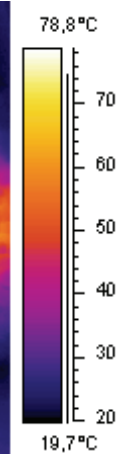

\section{Bild 2.7}
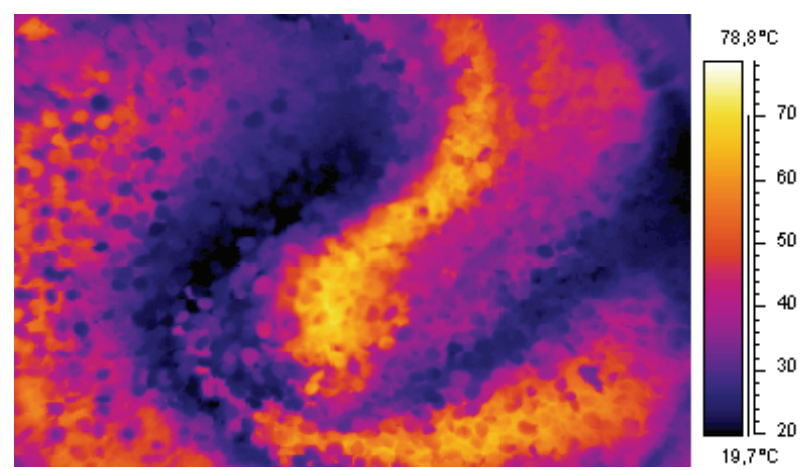

nfrarotaufnahme nach 1 Umdrehung Temperatur: $19,7-78,8^{\circ} \mathrm{C}$
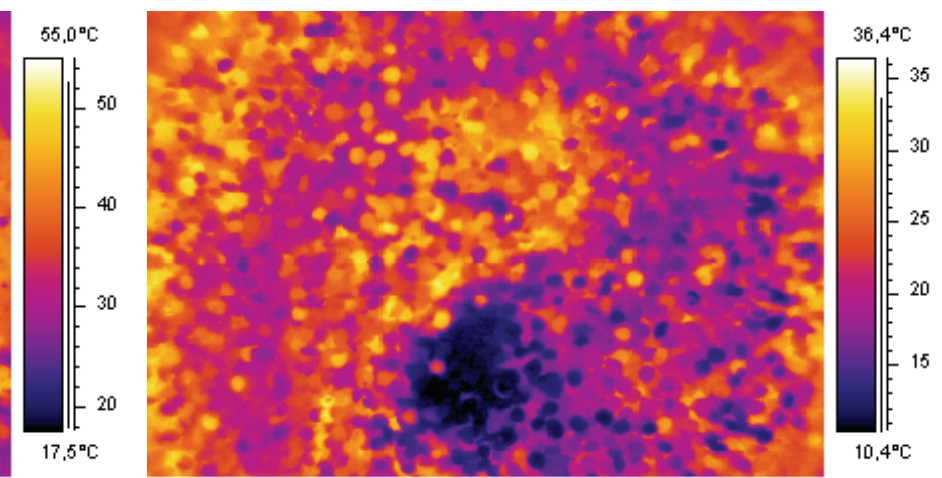

Bild 2.9: Infrarotaufnahme nach 10 Umdrehungen Temperatur: $10,4-36,4{ }^{\circ} \mathrm{C}$

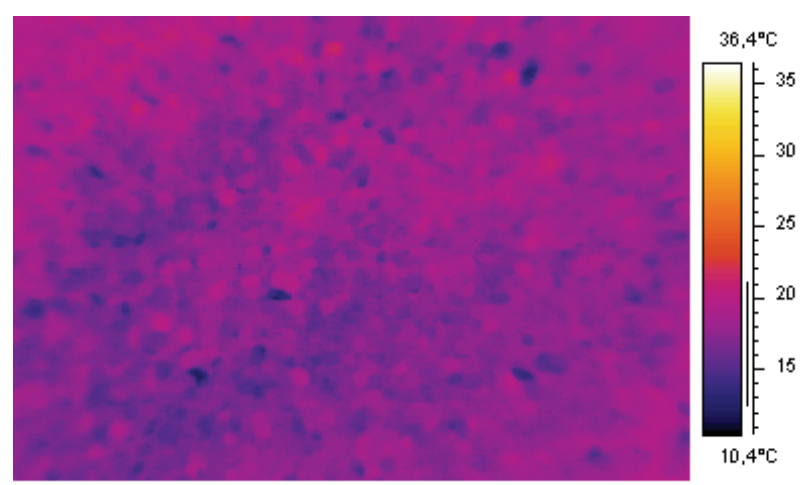


Das Temperaturniveau in Bild 2.10 ist erwartungsgemäß tiefer als in Bild 2.5, weil ein Temperaturausgleich zwischen den Körnern erfolgt ist.

\subsubsection{Versuch 2.2}

In Bild 2.11 sind die wesentlichen Ergebnisse des Versuches zur Körnermaistrocknung mit Warmluft grafisch dargestellt. Als Folge der Zulufttemperaturerhöhung nach 45 Minuten ist der Abluftfeuchtegehalt gesunken, wobei sich aber in Vorversuchen auch gezeigt hat, dass nach ca. einstündiger Trocknungsdauer mit vergleichbarem Eingangsfeuchtegehalt und weiterhin eingestellter Warmlufttemperatur von $95{ }^{\circ} \mathrm{C}$ der Abluftfeuchtegehalt aufgrund nachlassender Feuchtigkeitsabgabe des Körnermaises ebenfalls abfällt. Durch die Erhöhung der Zulufttemperaturen auf $130{ }^{\circ} \mathrm{C}$ hat sich mit einem Zeitverzug von 15 Minuten der Abluftfeuchtegehalt bei ca. $78 \%$ stabilisiert. Bis zum Ende der Trocknung ist der Abluftfeuchtegehalt auf etwa $35 \%$ gefallen. Die Ablufttemperatur hat von etwa $10{ }^{\circ} \mathrm{C} z u$ Versuchsbeginn auf $36,8^{\circ} \mathrm{C}$ zum Ende des Versuches zugenommen.

Die Korntemperatur konnte während der Trocknung nicht messen werden. Die größte Wasserbeladung der Abluft hat sich nach 90 Minuten Trocknungsdauer mit $20 \mathrm{~g} \mathrm{~m}^{-3}$ eingestellt, um danach bis zum Ende des Versuches auf ca. $14 \mathrm{~g} \mathrm{~m}^{-3}$ abzufallen. Der Wasserentzug hat sich im Verlauf der Trocknung deutlich verringert. Im Ergebnis sind $4300 \mathrm{~g}$ Wasser entzogen worden. Bei einem Anfangsgewicht der Körnermaisprobe von $25000 \mathrm{~g}$ (Feuchtegehalt 29 \%) betrug das Endgewicht der Probe 20700 g (Feuchtegehalt 14,2 \%). Die Trocknungsdauer betrug 210 Minuten. Bei einem thermischen Energieeinsatz von 8,38 kWh ergab sich hierdurch ein Trocknungsenergiebedarf von 1,94 $\mathrm{kWh} \mathrm{kg}^{-1}$ Wasserentzug. 


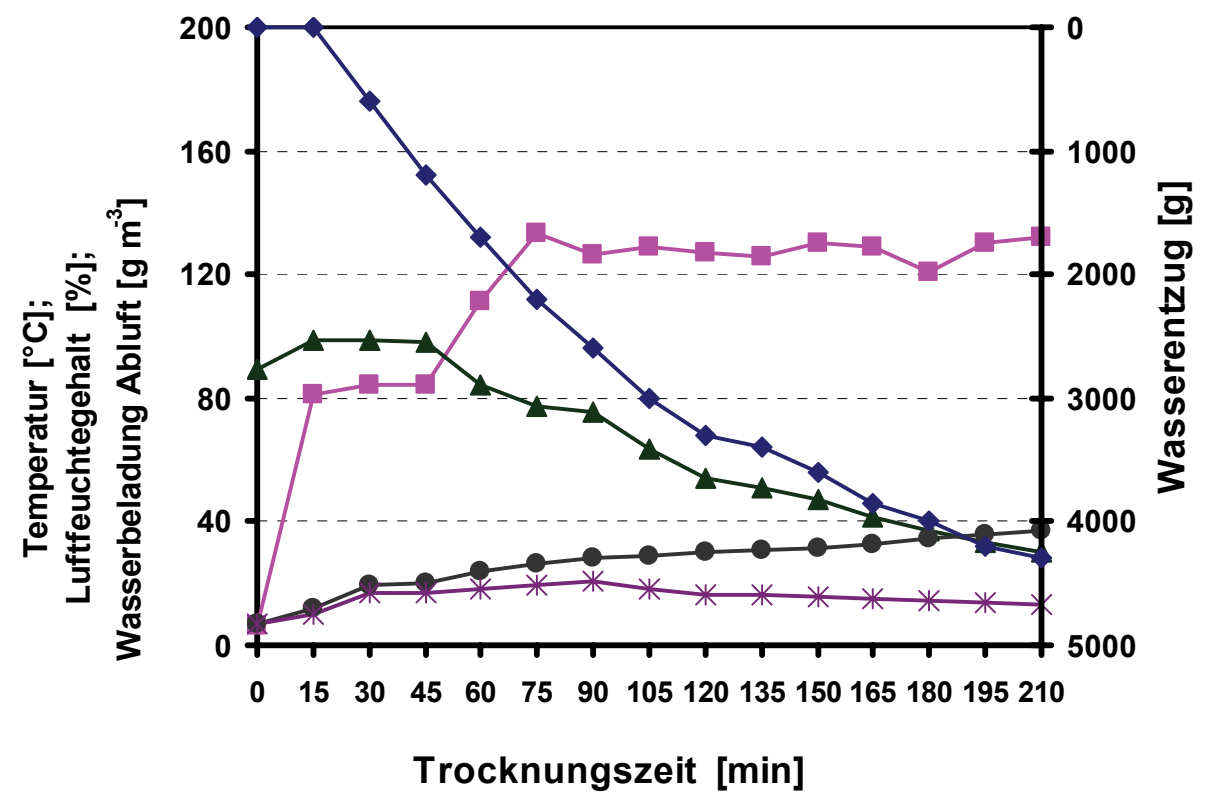

\section{$\rightarrow-$ Zulufttemperatur \\ $\rightarrow-$ Ablufttemperatur \\ $\multimap-$ Wasserentzug}

$\neg$ Abluftfeuchtegehalt

* Wasserbeladung Abluft

Bild 2.11: Warmlufttrocknung im Chargenmischertrockner

\subsubsection{Versuch 2.3}

Im Unterschied zu Versuch 2.2 wurde ergänzend zur Warmlufttrocknung Mikrowellenenergie unterstützend eingesetzt. Als Einschaltkriterium ist der Abluftfeuchtegehalt gewählt worden. Nach Unterschreiten von $75 \%$ rel. Feuchte wurde die Mikrowellenapplikation mit 360 W Sendeleistung für 30 Minuten eingeschaltet. Dieses war nach der 82. Trocknungsminute gegeben. Aufgrund dieser Mikrowellenzufuhr hat sich eine Erhöhung des Abluftfeuchtegehaltes auf ca. 80 \% eingestellt. Damit hat sich die Wasserbeladung der Abluft auf 28,1 $\mathrm{g} \mathrm{m}^{-3}$ erhöht. Zum Ende der Trocknung ist der Abluftfeuchtegehalt auf 19,3\% abgefallen. Der erforderliche Wasserentzug von $4300 \mathrm{~g}$ ist bereits nach der 135 . Trocknungsminute erreicht worden, die Ablufttemperatur erreichte zum Ende der Trocknung $45,9^{\circ} \mathrm{C}$. Bei einem Energieeinsatz von $6,15 \mathrm{kWh}$ für Warmluft und einem Energieeinsatz für den Mikrowelleneinsatz von 0,25 kWh ergab sich zum Trocknen eine eingesetzte Energie von $6,40 \mathrm{kWh}$. Der spezifische Trocknungsenergiebedarf betrug somit $1,48 \mathrm{kWh} \mathrm{\textrm {kg } ^ { - 1 }}$ Wasserentzug. 


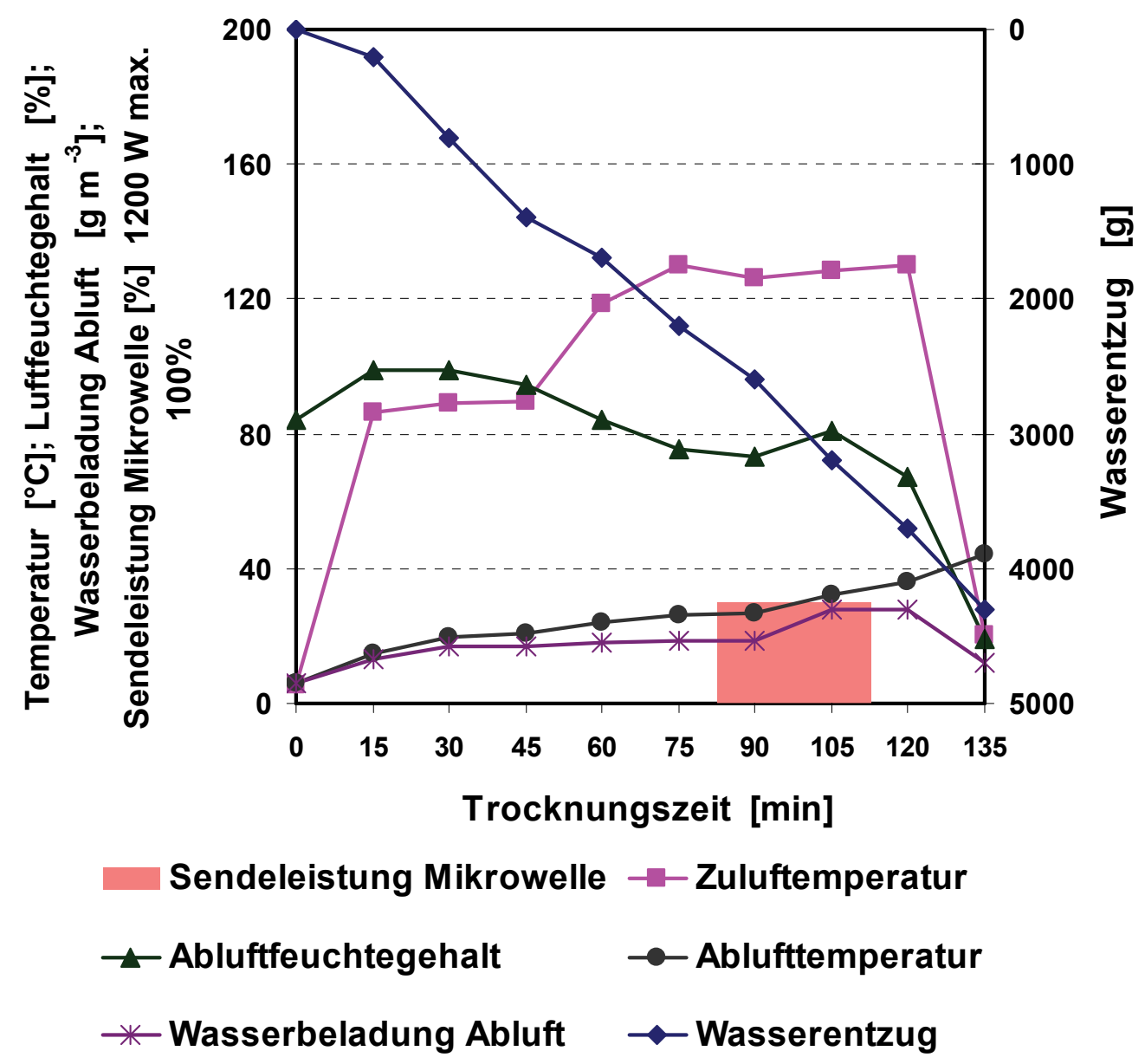

Bild 2.12: Mikrowellenunterstützte Warmlufttrocknung im Chargenmischertrockner

\subsection{Diskussion}

Bild 2.5 verdeutlicht eine typische Mikrowelleneigenschaft. Die Mikrowellen verteilen sich im Arbeitsraum nicht gleichmäßig. Dieses führt zu unterschiedlichen Absorptionen und damit zu ungleichen Erwärmungen, die sich in unterschiedlichen Temperaturen (hot spots) zeigen. Die Abstände zwischen heißen und kalten Stellen zeigen, dass sich eine stehende Welle ausgebildet hat, die durch die Geometrie des Trocknerinnenraumes, durch das Trocknungsgut und die Befüllhöhe beeinflusst worden ist. Hierdurch hervorgerufene Temperaturdifferenzen sind für Trocknungsvorgänge unerwünscht. Abhilfe ist mechanisch durch Umrühren der Schüttung (Bilder 2.6 bis 2.10) möglich. Eine andere Möglichkeit ist der Einsatz eines Feldrührers (Decareau 1986, Fanslow 1971), um das Mikrowellenfeld im Arbeitsraum zu vergleichmäßigen. Aus Versuch 2.1 zeigt sich, dass der hohe Feuchtigkeitsgehalt von Körnermais eine begrenzte Eindringtiefe der Mikrowellen bei guter Energieumsetzung ergibt. Zur Abfuhr der freigesetzten Feuchte ist nicht angewärmte Prozessluft eingesetzt worden, 
wobei aber durch die Strömungsrichtung bedingt Wärmeenergie in tiefere Kornschichten verschoben wurde. Hierdurch wurde das Temperaturprofil der Maisschüttung beeinflusst. Für gleichmäßige Korntemperaturen ist daher ein permanentes Durchmischen erforderlich. In Vorversuchen hat sich anhand der Zugabe von verfärbtem Körnermais gezeigt, dass bei der vorstehenden Versuchsanlage und auf Dauerbetrieb geschaltetem Rührwerk eine vollständige Durchmischung nach ca. 360 Sekunden (60 Rührwerksumdrehungen) gegeben war.

Der stündliche Wasserentzug ist bei der Körnermaistrocknung abnehmend (Mühlbauer 1974, Toftdahl 1987). Über den gesamten Trocknungsverlauf gesehen weisen praxistypische Warmlufttrockner bei der Maistrocknung von $35 \%$ auf $15 \%$ einen mittleren Feuchtentzug von ca. $4 \%$ pro Stunde auf. Hierzu ist ein Verhältnis von Inhalt im Trockenraum zu Durchsatz pro Stunde von 3,5:1 bis 4,5:1 erforderlich. Je niedriger der Anfangsfeuchtegehalt ist, desto geringer ist der durchschnittliche Feuchteentzug pro Stunde (Kröll und Kast 1989). Der Wasserentzug aus Versuch 2.2 erforderte 210 Trocknungsminuten von einem Anfangsfeuchtegehalt $29 \%$ auf einen Endfeuchtegehalt von 14,2\%. Das entspricht einem mittleren Feuchteentzug von 4,22\% $\mathrm{h}^{-1}$. Unter Berücksichtigung des Anfangsfeuchtegehaltes von $29 \%$ zeigt sich, dass die Bemessung des Chargenmischertrockners hinsichtlich möglicher Trocknungszeiten mit praxisbekannten Warmlufttrocknern korreliert.

Bei Versuch 2.2 beträgt der Trocknungsenergieeinsatz 1,94 kWh je kg Wasserentzug. Dieser Energiebedarf ist im Vergleich zu Warmlufttrocknern aus der Praxis hoch, wobei das Versuchsziel eine kurze Trocknungszeit zu erreichen sich auch nur dann realisieren lässt, wenn über die gesamte Trocknungsdauer ein ausreichend hohes Dampfdruckgefälle zwischen den Körnern und der Prozessluft besteht. Die Abluftsättigung ist mit fortschreitender Trocknung nachlassend. Das ist typisch für chargenweises Trocknen. In die Energiebetrachtungen fließt auch die Erwärmung des Trockners ein. Bezogen auf die Masse Feuchtmais von $25 \mathrm{~kg}$ ist die Eigenmasse mit 77,20 kg hoch. In der Praxis weisen aus Stahl, Edelstahl oder Aluminium gebaute Körnermaistrockner mit einem Fassungsvermögen von beispielsweise $40 \mathrm{t}$ Körnermais eine Eigenmasse von ca. $10 \mathrm{t}$ bis $15 \mathrm{t}$ auf. Bei dem Versuchstrockner sind die Masseverhältnisse umgekehrt. Die Trocknermasse ist deshalb von praktischer Bedeutung, weil sich trotz der Wärmeisolierung des zylindrischen Behälters eine Energieaufnahme der wärmeaufnehmenden Bauteile einstellt (Gerecke 1990). Eine Betrachtung der reduzierbaren Wärmeverluste soll aber in dieser Ausarbeitung nicht vertieft werden, der Einfluss auf die Versuche 2.2 und 2.3 ist vergleichbar. Bedingt durch den Anfangsfeuchtegehalt von $29 \%$ ist der Wärmeeintrag in die Maiskörner durch eine schlechtere Wärmeleitung langsamer, als bei 
einem Anfangsfeuchtegehalt von $35 \%$. Hierdurch wird ein Grund für den hohen spezifischen Energiebedarf bei dieser Versuchsdurchführung gesehen. Durch weitere Versuchsreihen während der Erntezeiträume im Herbst 2003 bis 2006 mit diesem Trockner hat sich aber gezeigt, dass mit reiner Warmlufttrocknung und Eingangsfeuchten von 35 Prozent ein spezifischer Energiebedarf von $1,15 \mathrm{kWh} \mathrm{kg}^{-1}$ Feuchtentzug ausreicht, allerdings ist dann etwa die doppelte Trocknungszeit erforderlich. Bei der Warmlufttrocknung besteht zwischen minimalem Energieeinsatz und maximalem Durchsatz ein Zielkonflikt.

Der Entzug von 29 auf $14 \%$ Feuchtegehalt erfordert bei mikrowellenunterstützter Warmlufttrocknung (Versuch 2.3) bei gleich eingestellten Prozessluftbedingungen eine Trocknungsdauer von 135 Minuten (2,25 Std.), wobei auch der Energiebedarf auf 1,48 kWh $\mathrm{kg}^{-1}$ Feuchteentzug deutlich abfällt. Im Vergleich von Bild $2.11 \mathrm{zu}$ Bild 2.12 zeigt sich, dass bis zur 75 Trocknungsminute die Kurvenverläufe vergleichbar sind. In Versuch 2.2 war aber das Niveau der Wasserbeladung deutlich geringer als in Versuch 2.3. Infolge dessen wurde der Wasserentzug von $4300 \mathrm{~g}$ deutlich früher erreicht. Hierdurch begründet sich die kürzere Trocknungsdauer. Eine kurze Trocknungszeit stellt einen großen trocknungstechnischen Vorteil dar, wobei durch die Mikrowellenapplikation nur soviel Wärmeenergie im Korninneren wirksam wird, wie es für einen bestimmten Feuchtentzug erforderlich ist. Die Mikrowellenenergie ist in Versuch 2.3 nach thermodynamischen Überlegungen für 30 Minuten zugeschaltet worden mit dem Ziel, die Trocknungsgeschwindigkeit $\mathrm{zu}$ erhalten und gegebenenfalls zu erhöhen. Der Energieanteil der Mikrowellenerwärmung betrug bei diesem Versuch 3,9 Prozentpunkte. Der Versuch 2.3 zeigt im Vergleich zum Versuch 2.2 einen signifikanten Anstieg der Ablufttemperatur zum Trocknungsende. Im Ergebnis ist bei dem mikrowellenunterstützten Versuch zuviel Energie an das Trocknungsgut herangeführt worden.

Eigene Vorversuche haben gezeigt, dass das Korn bei der gleichzeitigen Trocknung mit Warmluft und Mikrowellen eine höhere Temperatur annimmt, als bei gleicher Trocknungszeit und reiner Warmlufttrocknung mit gleicher Warmlufttemperatur. Bei optimierter mikrowellenunterstützter Prozessführung ist $\mathrm{zu}$ erwarten, dass es möglich ist, die Korntemperatur zum Trocknungsende niedriger zu halten als bei der Warmlufttrocknung. Dieses begründet sich damit, dass die Mikrowelle im Korninnern in Wärme umgewandelt wird und dort einen Dampfdruck erzeugt. Dadurch wird die Feuchtigkeit nach außen transportiert, wobei die Poren feucht bleiben. Warmlufttrockner können diesen Effekt prinzipbedingt nicht vollständig nutzen. Die Warmlufttrocknung bewirkt hingegen eine Trocknung zunächst an der Oberfläche, womit die Diffusion der Feuchtigkeit aus dem Korninnern erschwert wird. Durch 
Wärmeleitung wird die Wärme ins Korninnere transportiert, dort erhöht sich dann der Dampfdruck. Die Feuchtigkeit bewegt sich gegen stärker geschlossene Porenöffnungen. Um die Feuchtigkeit aus dem Korn abzuführen wird demzufolge eine höhere Temperatur benötigt. Es ist noch darzulegen, ob und inwieweit ein gezieltes oder über den Trocknungsverlauf getaktetes Aktivieren der Mikrowellen mit einer angepassten Sendeleistung den Trocknungsvorgang weiter beschleunigt, um dabei auch den Gesamtenergieeinsatz zu optimieren (Kialbekov et al. 2006, Radajewski et al. 1988, Shivhare et al. 1994).

\subsection{Schlussfolgerungen}

Die Versuche zielen darauf ab, die technische Machbarkeit einer Verfahrenskombination zur Trocknung von Körnermais mit Warmluft und Mikrowellen zu erarbeiten. Wesentliche Ziele sind dabei, Zusammenhänge zwischen Trocknungszeit und spezifischem Energiebedarf aufzuzeigen. Die Versuchsergebnisse zeigen, dass durch eine angepasste dielektrische Trocknung der Anteil konvektiver Energie reduziert werden kann. Im Vergleich zur reinen Warmlufttrocknung führt bei einem Parallelversuch die Verfahrenskombination mit einer mikrowellenunterstützten Warmlufttrocknung zu einer Verkürzung der Trocknungszeit um 35 Prozentpunkte und zu einer Reduzierung des Gesamtenergieeinsatzes um 22,6 Prozentpunkte. Damit die Mikrowellenenergie gleichmäßig im Produkt wirkt, ist eine ausreichende Produktbewegung während der Trocknung erforderlich. Weiterhin ist es vorteilhaft, wenn die Wirkrichtung der Mikrowellenergie mit dem Warmluftstrom gleichgerichtet ist. Durch eine richtige Auslegung der Trocknerkonstruktion, der Energiekreisläufe bestehend aus Wärmezufuhr zum Trocknungsgut, Abwärmenutzung der/des Magnetron(s) und Abwärmenutzung des erwärmten trockenen Gutes lassen sich Trocknungsreserven mobilisieren. Für eine optimale Anlagenkonfiguration ist es daher richtig, unterschiedliche Vorgänge während der Konservierung mit dem Oberbegriff Trocknen zu trennen. Hierbei ist die konstruktive Gestaltung der Mikrowelleneinkopplung in das Trocknungssystem wichtig. Bei guter Prozessführung wird auch der Trocknungsprozess seinerseits durch Optimierungen innerhalb der einzelnen Trocknungsabschnitte einschließlich der Rückkühlung und Wärmerückgewinnung verfahrenstechnisch unterschiedlich gestaltet sein müssen. Hier kann die ergänzende Mikrowellenapplikation die Konvektionstrocknung unterstützen. Bei der Weiterentwicklung zu praxisreifen Systemen wird der Einsatz zunehmend knapper und teurer werdender Energie an Trocknungsanlagen neu zu bewerten sein. 


\section{Teil}

\section{Einfluss von mikrowellenunterstützter Warmlufttrocknung bei der Körnermaistrocknung - Visualisierung mit dreidimensionaler Mikro- Computertomographie}

\section{Zusammenfassung}

Die Möglichkeiten und Grenzen der reinen Warmlufttrocknung sind auf der Bewertungsgrundlage nach thermodynamischen Grundsätzen bekannt. Züchterische Fortschritte und steigende Erträge fordern verfahrenstechnische Anpassungen zur Konservierung durch Trocknung, um Korninhaltsstoffe optimal zu erhalten. Gleichzeitig sind aber die Prozesse, die bei der Trocknung im Korninnern stattfinden noch nicht vollständig bekannt. Im Hinblick darauf wurde am Department für Agrartechnik der Universität Göttingen und im Labor für Mikrowellentechnik am Institut für Technologie- und Wissenstransfer, TWS, im Kreis Soest e.V. untersucht, wie eine Verfahrenskombination aus Warmluft- und Mikrowellentrocknung auf Körnermais wirkt. Die Untersuchungen hierzu erfolgen mit Hilfe dreidimensionaler Mikro-Computertomographie. Die Ergebnisse zeigen auf, dass unterschiedliche Trocknungsintensitäten zu verschiedenen geometrisch-plastischen Form- und Volumenänderungen führen. 


\subsection{Einleitung}

Die Kombination von Warmlufttrocknung mit unterstützter Mikrowellenapplikation ist eine Möglichkeit, die Trocknung $\mathrm{zu}$ beschleunigen, wobei unter Beachtung einer verfahrenstechnisch richtigen Prozessführung die Trocknungsdauer deutlich verkürzt werden kann (Wieneke 1988). Gleichwohl ist der Einsatz von Mikrowellenenergie eine gute Möglichkeit, die Grenzen der maximalen Trocknungsgeschwindigkeit von Körnermais überhaupt zu ermitteln.

Es besteht weiterhin Entwicklungsbedarf, um die Ziele zur Erhaltung einer hohen, auf die Verwendung ausgerichteten Produktqualität bei möglichst kurzen Trocknungszeiten zu vereinen. Bisherige Untersuchungen stützten sich neben der Untersuchung der Korninhaltsstoffe nur auf die äußeren kornspezifischen Parameter zur Beurteilung einer möglichen und zulässigen Trocknungsgeschwindigkeit.

\subsection{Stand der Technik}

Bisherige zweidimensionale mikroskopische Aufnahmen sind bei getrockneten Maiskörnern kaum anwendbar, weil Mikrotomschnitte aufgrund der Kornstruktur keinen planen Schnitt ermöglichen (Gerlach 1985, Nultsch \& Grahle 1983). Zudem gelingt dieses nicht zerstörungsfrei. Bei einer geringen 10-fachen Vergrößerung eines zuvor geschnittenen Maiskorns ist die Gesamtstruktur zwar erkennbar, Rückschlüsse auf Vorgänge während der Trocknung lassen sich daraus nicht schlüssig ableiten.
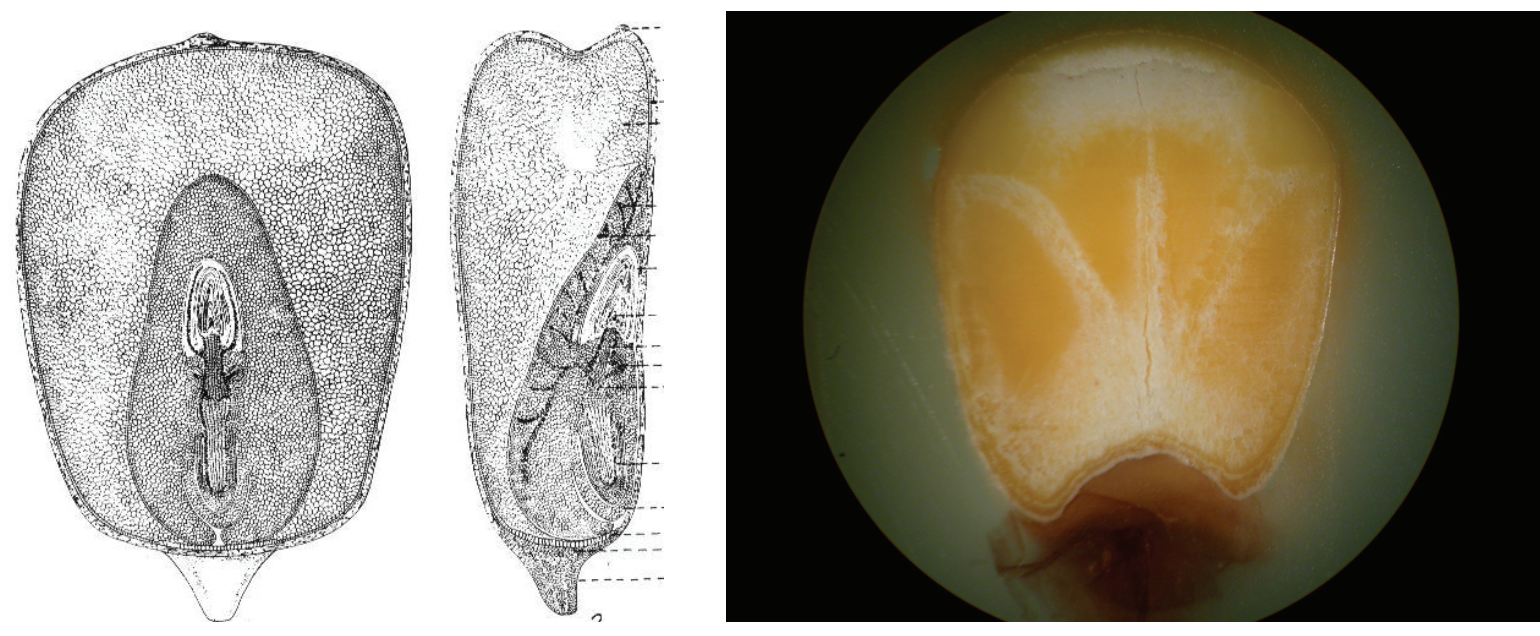

Bild 3.1: Abbildung Maiskorn im Schnitt Bild 3.2: geschnittenes Maiskorn (Zscheischler et al. 1990) Mikroskopaufnahme $(10 \mathrm{x})$ 
Fragen nach Vorgängen zum Feuchteaustritt an Maiskörnern können nach Wieneke (1972) und Mühlbauer (1974) dadurch visualisiert werden, indem Maiskörner in ein Ölbad eingetaucht werden, über dem ein Unterdruck erzeugt wird. Infolge des geringen Drucks, beginnt die Feuchte im Korn bereits bei Raumtemperatur zu sieden. An der Oberfläche des Maiskorns treten Dampfblasen aus. Zu Beginn der Trocknung erfolgt der Austritt der Blasen hauptsächlich an der Ansatzstelle des Korns am Kolben, mit fortschreitender Trocknung an der gesamten Kornoberfläche. Bei der Trocknung mit hohen Lufttemperaturen kann der im Innern des Korns entstehende Wasserdampf nicht in dem Maße durch die Zellwände diffundieren in welchem er entsteht, so dass sich eine Dampfblase im Korninnern ausbildet und die Feuchtebewegung durch eine hydrodynamische Strömung verstärkt wird. Der Druck im Korninnern kann Werte annehmen, bei denen die Zellwände der Belastung nicht mehr standhalten und die Körner platzen. (Mühlbauer 1974). Wenn im Korninnern Dampfdruck durch Mikrowellenenergie aufgebaut wird, kann prinzipiell der gleiche Zustand entstehen.

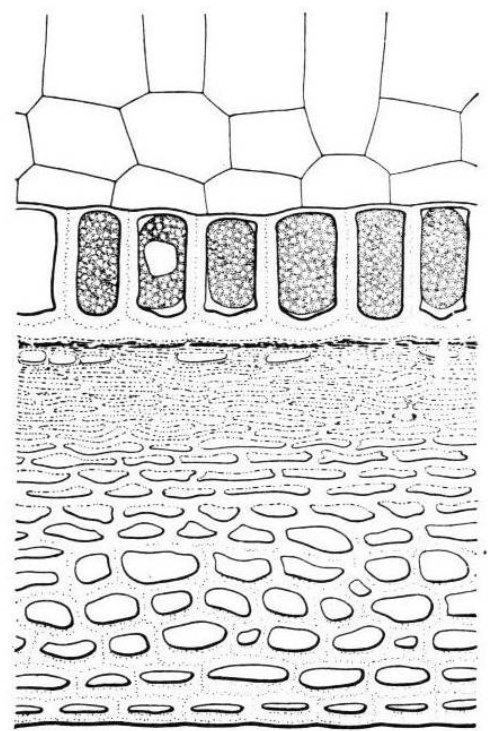

Bild 3.3: Querschnitt durch den Schalenbereich des Maiskorns (Gassner et al. 1989)

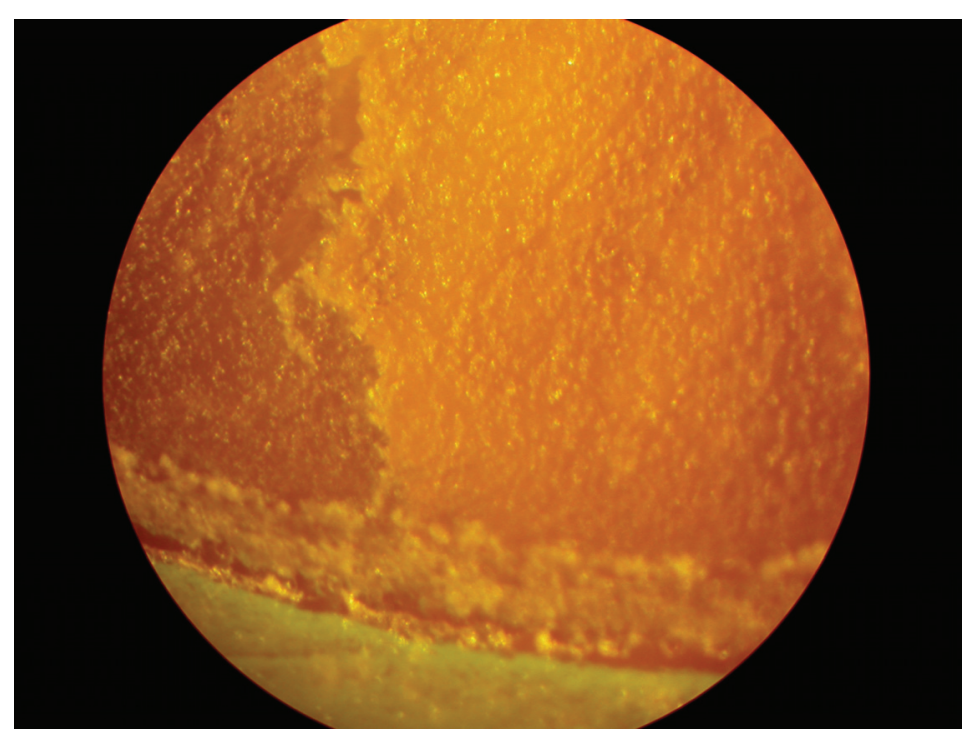

Bild 3.4: Mikroskopische Aufnahme vom Schalenbereich eines Maiskorns (40 x).

Mühlbauer (1974) führt aus, dass die aus früheren Untersuchungen getroffene Annahme, dass die Schale der Feuchtebewegung einen erheblichen Widerstand entgegensetzt, bei Trocknungsversuchen nicht bestätigt werden konnte. Körner mit und ohne Schale weisen das gleiche Trocknungsverhalten auf.

Quantitative Untersuchungen über die Feuchtebewegungen im Korn und deren Ursachen sind auf Grund der komplizierten Struktur der Maiskörner nicht möglich. Mühlbauer (1974) stellt 
fest, dass die Fluoreszensmethode zur Sichtbarmachung der Feuchteverteilung in Maiskörnern nicht anwendbar ist. Bei dem Versuch einer Klärung der Mechanismen der Feuchtebewegung im Korn ist man auf Beobachtungen am trockenen Korn angewiesen. $\mathrm{Zu}$ mikroskopischen Zwecken werden von Gassner et al. (1989) und Robenek (1995) verschiedene Färbemethoden biologischer Proben beschrieben, um deren Art und Zusammensetzung sowie auch eine Einschätzung der Feuchteverteilung $\mathrm{zu}$ erhalten. Bei der Färbung ist zwischen zwei Reaktionsmöglichkeiten zu unterscheiden. Entweder lagert sich der Farbstoff adsorptiv an die zu färbende Substanz bzw. Struktur an oder der Farbstoff geht eine kovalente Bindung mit der zu färbenden Substanz ein (Robenek 1995).

Versuche zu durch Trocknungsenergie verursachten räumlichen Temperaturverteilung im Korn sind durch den Einsatz spezieller Mikrothermometer möglich, sie erlauben jedoch nur eine grobe Beurteilung der nachhaltigen Wirkung von Trocknungswärme auf das Korn. Mühlbauer (1974) hat hierzu in zu trocknende Maiskörner Thermoelemente eingebracht, die ohne Zwischenraum auf ein Mantelthermoelement aufgefädelt worden sind. Am Messkorn ist die Schale an der Ansatzstelle durchstochen worden und das Thermoelement wurde eingepresst.

Mühlbauer (1974) stellt fest, dass die nach obigem Verfahren gemessenen Korntemperaturen lediglich Näherungswerte darstellen, da das Anbringen der Thermoelemente an exakt definierten Stellen des Korns bei dem inhomogenen Kornaufbau nicht möglich ist.

Zerstörungsfreie Untersuchungen an Maiskörnern sind im Wesentlichen sensorische Prüfungen hinsichtlich Farbe, Konsistenz, Form, Geruch und dem spezifischen Gewicht. Die Korntemperatur (innere Temperatur) lässt sich nur im Gleichgewichtsfeuchtezustand bestimmen, wobei diese vorteilhaft in einer homogenen Kornschüttung feststellbar ist. Die Oberflächentemperatur lässt sich hingegen mit Infrarotmesstechnik oder einem Kontaktthermometer regelmäßig messen. Ganzkornfeuchtemessgeräte erlauben auch mit Hilfe eines kapazitiven Messverfahrens das zerstörungsfreie Bestimmen des Gutfeuchtegehaltes, wobei hierzu auch der Gleichgewichtsfeuchtezustand und eine homogene Korntemperaturverteilung bestehen müssen. Mühlbauer (1974) führt aus, dass ein Ausmessen der Kornoberfläche oder Volumenberechnungen an Maiskörnern aufgrund der unterschiedlichen Formen und Abmessungen erhebliche Schwierigkeiten bereitet.

Untersuchungstechniken zu Zuständen oder der Struktur im Korninnern sind begrenzt. Eine Möglichkeit hierzu ist die Anwendung der Röntgentechnik, wie sie in der Humanmedizin 
bekannt ist (Friedmann et al. 1981), aber zur Beurteilung pflanzlicher Organismen bislang wenig eingesetzt wird. Die Röntgentechnik ermöglicht den zerstörungsfreien Blick in das Innere von Maiskörnern, die räumliche Zuordnung von Gewebeteilen und deren exakte Vermessung mit einer maximalen Auflösung von $10 \mu \mathrm{m}$. Eine Tiefeninformation ist dabei nicht vorhanden. Anders als bei der zweidimensionalen Röntgentechnik, in der die Tiefeninformation verloren geht, erlaubt die rechnergestützte Volumenkonstruktion mit Hilfe einer rotierenden Probe erstmals einen dreidimensionalen Bildaufbau des Maiskornes. Im Vergleich zur zweidimensionalen Röntgentechnik stellen sich Unterschiede der stärker strahlenabsorbierenden Frucht- und Samenschalen aussagefähiger dar. Als weltweit erstes und bislang einziges Pflanzenzuchtunternehmen hat die STRUBE-DIECKMANN GmbH und Co. KG in Zusammenarbeit mit dem Entwicklungszentrum für Röntgentechnik der Fraunhofer Gesellschaft ein hochauflösendes dreidimensionales Röntgensystem entwickelt. Aufbauend auf die Versuchsergebnisse zur Warmlufttrocknung mit Mikrowellenapplikation (Teil 2) wird untersucht, wie im Gegensatz zur Trocknung unter weitgehend natürlichen Bedingungen (20 ${ }^{\circ} \mathrm{C}$ und $65 \%$ rel. Luftfeuchtegehalt) kornphysikalische Änderungen bei der zur Futtermaisgewinnung bewährten Warmlufttrocknung mit einer Trocknungslufttemperatur von ca. $125{ }^{\circ} \mathrm{C}$ entstehen und welche Vorgänge deutlich werden, wenn der Trocknungsenergieeintrag durch Mikrowellen erhöht wird. Es stellt sich daher die Frage, bis zu welchen Verhältnissen von Warmluft und Mikrowellenenergie die Trocknungsgeschwindigkeit gesteigert werden kann und welche Auswirkungen sich auf das Trockengut und den spezifischen Trocknungsenergiebedarf ergeben. Hieraus ergibt sich auch die Frage, ob unterschiedliche Trocknungsintensitäten Einfluss auf die Gleichgewichtsfeuchte bzw. das Sorptionsverhalten haben.

\subsection{Material und Methoden}

\subsubsection{Aufbau der Versuchstrocknungsanlage}

Für die nachfolgend beschriebenen Versuche ist der unter 2.3.1 (Aufbau der Versuchsanlage) beschriebene und abgebildete Chargenmischertrockner (Teil 2) mit gleicher Ausstattung eingesetzt worden. 


\subsubsection{Aufbau der Mikrocomputertomographieanlage}

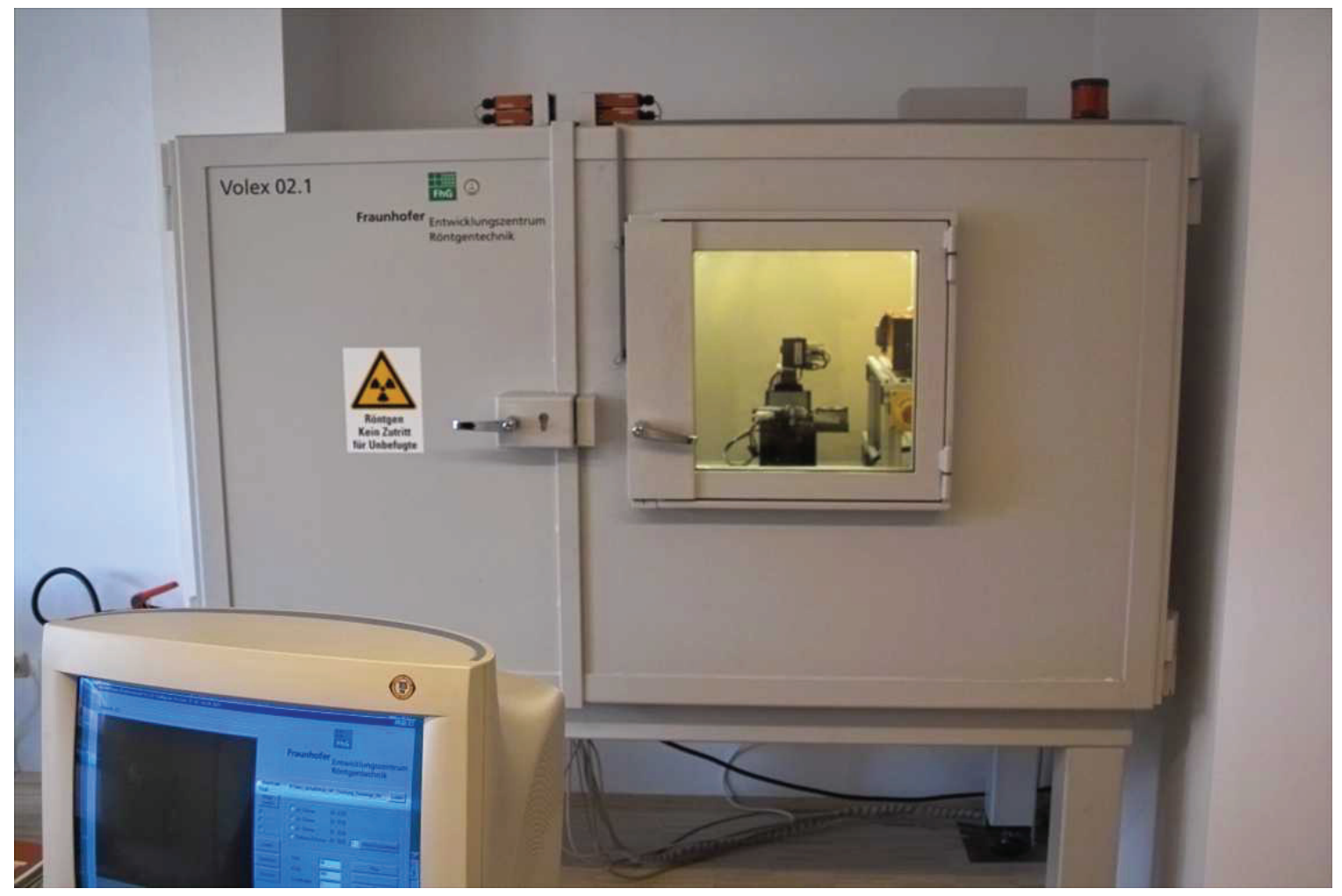

Bild 3.5: Ansicht auf die CT-Röntgenkabine im Betriebszustand

Bild 3.5 zeigt die im Hause Fr. Strube Saatzucht GmbH \& Co. KG in Söllingen installierte Röntgenkabine. Auf Grund der relativ geringen Röntgen Absorption der biologischen Objekte ist eine Maximalenergie der verwendeten Röntgenstrahlung von $150 \mathrm{kV}$ ausreichend. Die Röntgenkabine ist mit einem Objektmanipulator mir vier motorisierten Präzisionsachsen ausgestattet. Diese gestatten die genaue Einstellung der gewünschten Vergrößerung und die automatische Justage der Objekt-Drehachse. Mit der eingesetzten Hardware, Röntgenröhre mit Mikrofokustechnik und Flat-Panel-Detektor mit 512² (=262144) Einzelelementen ist es möglich, Auflösungen bis zu $10 \mu \mathrm{m}$ zu erreichen. In dem System sind zwei Rechner vorhanden. Der eine Rechner übernimmt die Steuerung und Aufnahme, während der andere mit Hilfe eines entsprechenden Algorithmus die Online-Rekonstruktion des untersuchten Volumens durchführt. 


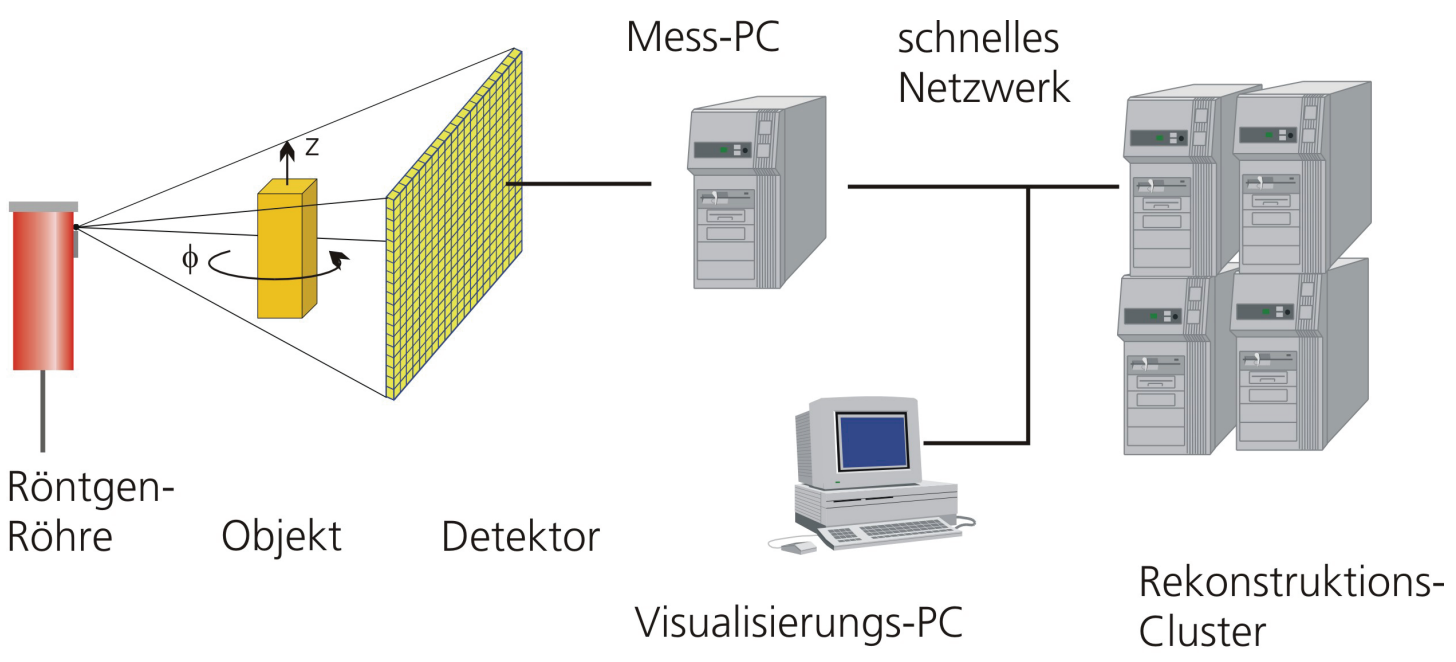

Bild 3.6: Prinzipieller System-Aufbau der 3D-Computertomographie

In Zusammenarbeit mit dem Hause Fr. Strube Saatzucht GmbH \& Co. KG und dem Institut für Technologie und Wissenstransfer, Soest (TWS) wurde am Standort der Röntgenanlage in Söllingen zunächst eine Aufnahmeapparatur aus Styropor entwickelt, um die bislang nur für Rübensamen genutzte Anlage für die deutlich größeren Maiskörner einsetzen zu können. Nach der Auswertung von Vorversuchen wurde ein Körnermaisaufnahmeträger für die Aufnahme von 12 Maiskörnern gefertigt. Diese 12 Körner können dann innerhalb eines Röntgendurchganges untersucht werden.

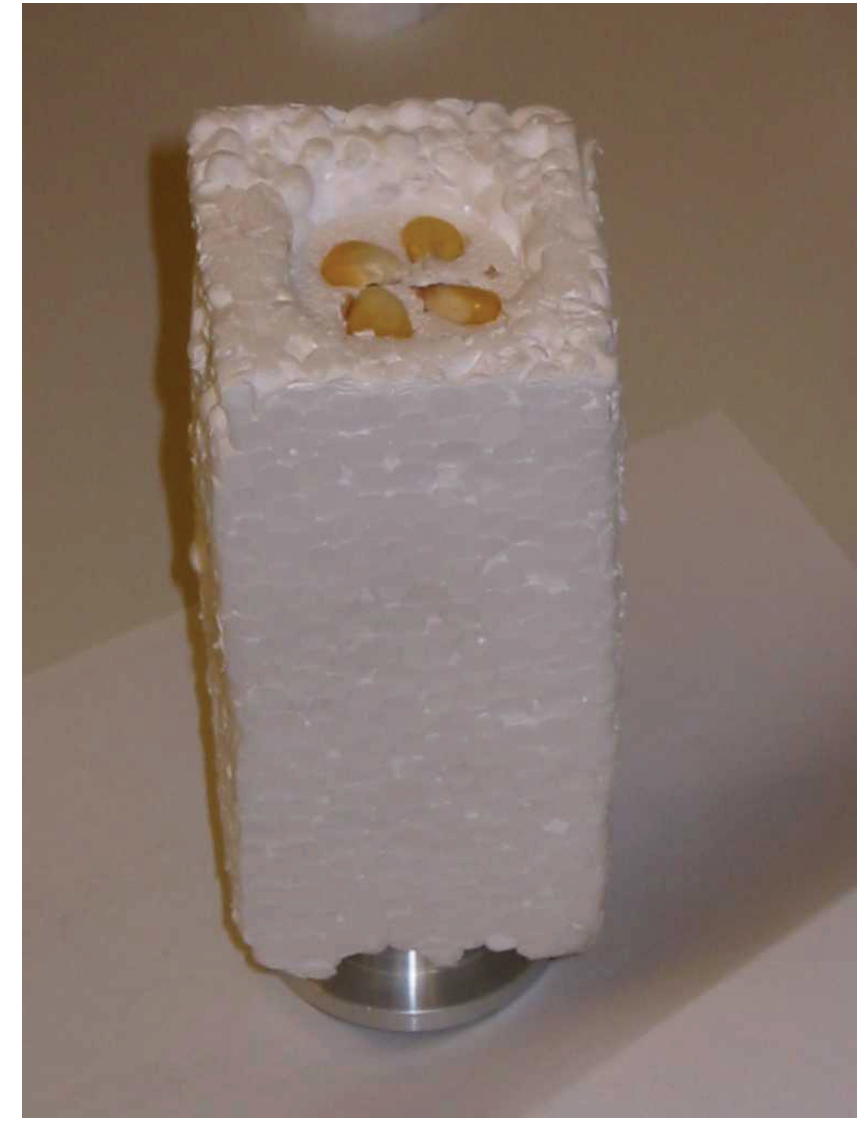

Bild 3.7: Einbettung der Maisproben in den Probeträger aus Styropor (jeweils vier Maiskörner in drei Ebenen) 


\subsubsection{Beschreibung der Sorptionsversuche}

Körnermais ist ein hygroskopisches Gut, das heißt, der Wassergehalt des Gutes passt sich an den relativen Feuchtegehalt der umgebenden Luft an. Dabei stellt sich jeweils ein hygroskopisches Gleichgewicht ein. Im hygroskopischen Gleichgewichtszustand wird die Beziehung zwischen Wassergehalt und Gleichgewichtsfeuchtegehalt des Produktes durch Sorptionsisothermen ausgedrückt. Für jeden Feuchtegehalt der umgebenden Luft zeigt eine Sorptionsisotherme den entsprechenden Wassergehalt des Materials bei einer gegebenen, konstanten Temperatur an. Ändert sich die Zusammensetzung des Materials, so ändert sich auch das Sorptionsverhalten. Bedingt durch die Komplexität der Sorptionsvorgänge können Isothermen nicht rechnerisch, sondern nur experimentell ermittelt werden (Kersten 2004).

Ziel der nachfolgend beschriebenen Sorptionsversuche ist es nicht, vollständige Sorptionsisothermen für den untersuchten Körnermais zu entwickeln. Im Vordergrund stehen Untersuchungen, ob unterschiedliche Trocknungsparameter Einfluss auf das Sorptionsverhalten haben. Der Versuchsaufbau hierzu ist aber prinzipiell vergleichbar, nicht aber die graphische Ergebnisdarstellung.

Für die Bestimmung des Sorptionsverhaltens der Körnermaisproben nach der Trocknung ist ein Klimaschrank eingesetzt worden. Der Klimaschrank wird durch einen Thermostat und Hygrometer überwacht, diese erlauben eine Temperaturkonstanz von +/- $1 \mathrm{~K}$ und eine Feuchtekonstanz von +/-1 \% rel. Luftfeuchte. Für die Körnermaisproben stehen aus einem Drahtgewebe bestehende Probenträger zur Verfügung. Der Klimaschrank ist für das Einstellen der Gleichgewichtsfeuchte aller Proben erforderlich, bevor das Adsorptions- bzw. Desorptionsverhalten ermittelt werden kann. Weiterhin ist eine entsprechende Anzahl verschließbarer und auch belüftbarer Behälter erforderlich, welche die Aufrechterhaltung gleichbleibender Klimabedingungen sowie eine Gewichtsüberprüfung ermöglichen. Die Einstellung der relativen Luftfeuchte in den Versuchsbehältern erfolgte über Wasser und gesättigte Salzlösungen. Über der Lösung stellt sich in Abhängigkeit von der Temperatur eine bestimmte Luftfeuchte in den geschlossenen Behältern ein. 
Tabelle 3.1: Relative Feuchten über gesättigten Salzlösungen (Römpp 1995)

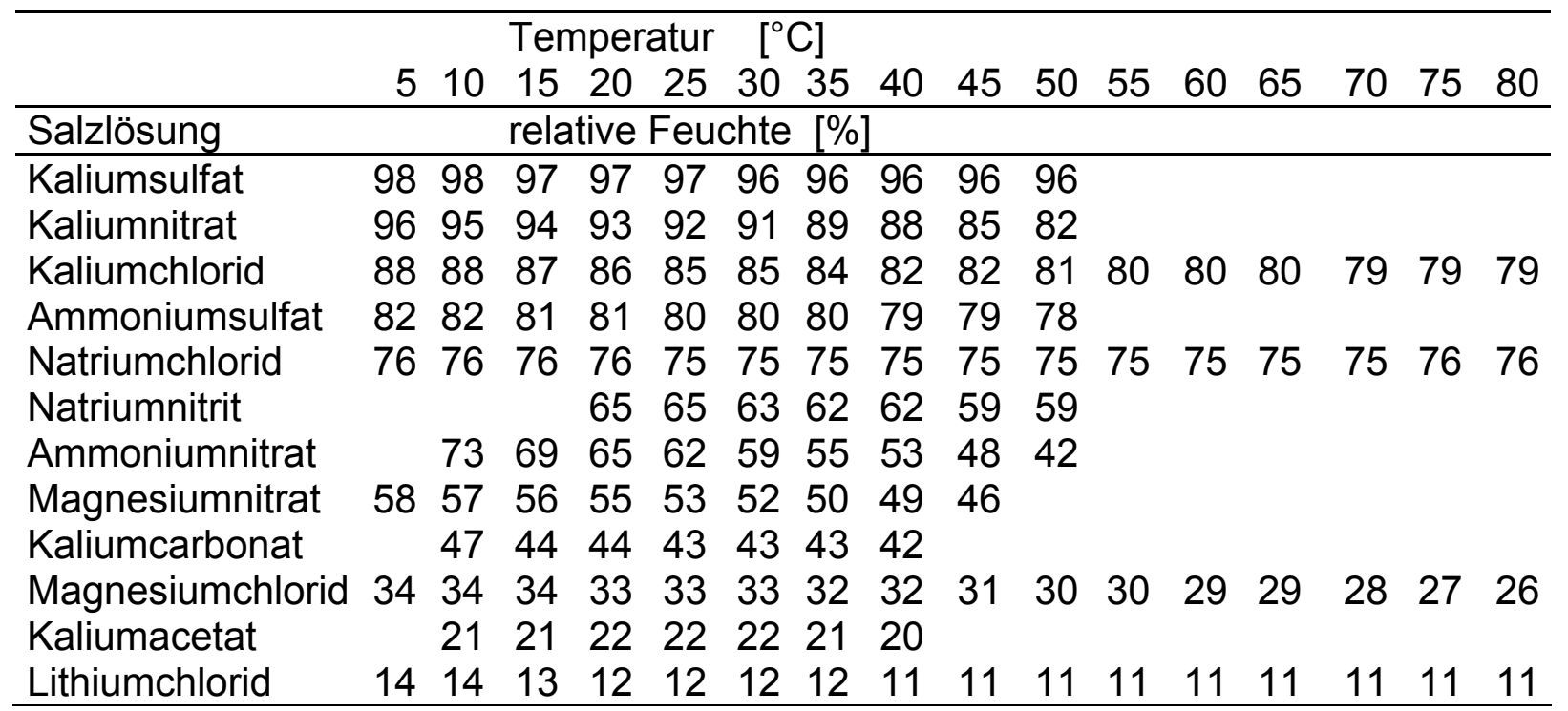

Versuche zum Adsorptionsverhalten sind mit Ammoniumsulfat $\left(\left(\mathrm{NH}_{4}\right)_{2} \mathrm{SO}_{4}\right)$ durchgeführt worden, zum Desorptionsverhalten ist Magnesiumchlorid $\left(\mathrm{MgCl}_{2}\right)$ eingesetzt worden.

Diese Methode ist bei der Bestimmung von Sorptionsisothermen für Lebensmittel und landwirtschaftliche Produkte (Bild 3.9) sowie bei der Kalibrierung elektrischer Feuchtefühler gebräuchlich (Nemitz 1963, Weber 1995). Die unbelüfteten Körnermaisproben wurden in Glasgefäße mit einem Inhalt von 31 eingebracht (Bild 8). Auf einem Ständer (c) befand sich etwa $6 \mathrm{~cm}$ von der Flüssigkeitsoberfläche entfernt ein Probenträger (d), der eine bestimmte Körnermaismasse enthielt. In den im Verhältnis zur Probenmasse großvolumigen Behältern erfolgt aufgrund von Dichteunterschieden eine geringe Luftzirkulation, die das Einstellen der Gleichgewichtszustände der Körnermaisproben unterstützt. 
a Glasgefäß

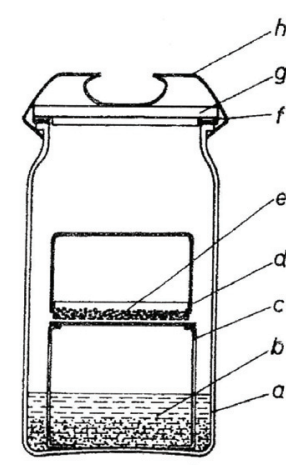

(3 1 Fassungsvermögeri)

b Salzlösung

c Ständer mit

gelochter Tragplatte

d Probenträger

e Körnermaisprobe

f Gummidichtring

g Glasdeckel

h Verschlussklammer

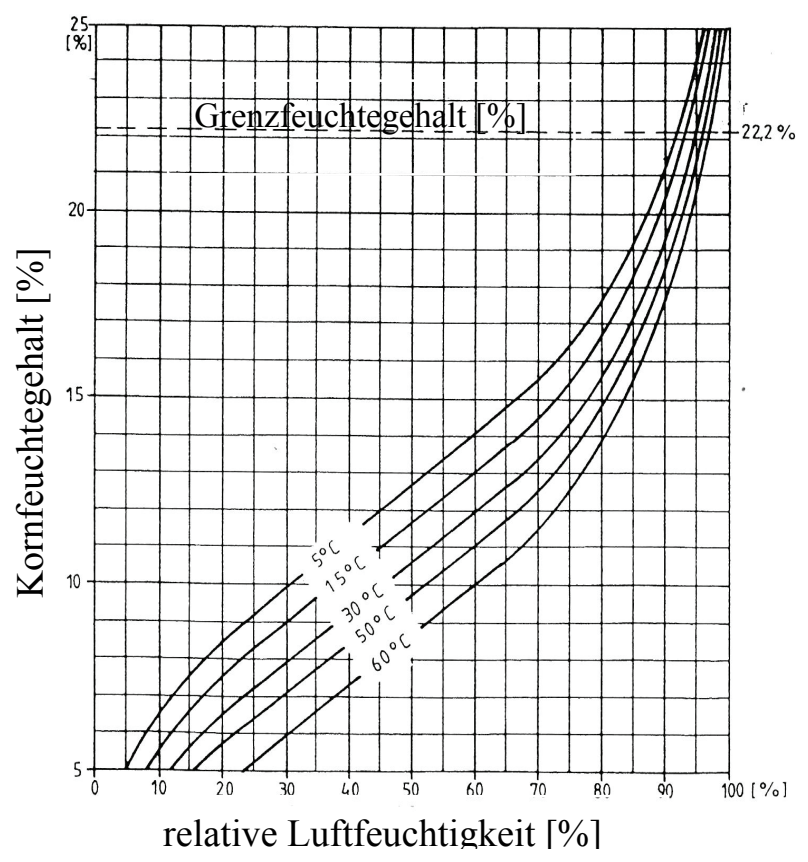

Bild 3.8: Versuchsgefäß zur Ermittlung des Sorptionsverhaltens einer Körnermaisprobe in unbewegter Luft.

Bild 3.9: Sorptionsisothermen von Mais (Goldsaat 1988)

Weitere, zu dieser Versuchsanordnung gehörende Ausstatiungen sind eine Feinwaage mit einer Auflösung von $0,001 \mathrm{~g}$ sowie ein Exsikkator, dessen Sohle mit wasserfreiem Kalziumsulfat befüllt ist.

\subsubsection{Versuchsdurchführung}

\subsubsection{Körnermaistrocknung}

Aufbauend auf unter 2.3.2 beschriebene Versuchsdurchführung zur Warmlufttrocknung mit Mikrowellenapplikation in dem Chargenmischertrockner (Teil 2) soll untersucht werden, wie im Gegensatz zur korntypischen Trocknung unter weitgehend natürlichen Bedingungen $\left(20^{\circ} \mathrm{C}\right.$ und $65 \%$ rel. Luftfeuchtegehalt) kornphysikalische Änderungen bei der zur Futtermaisgewinnung bewährten Warmlufttrocknung mit einer Trocknungslufttemperatur von ca. $125{ }^{\circ} \mathrm{C}$ entstehen, und ob weitere Vorgänge deutlich werden, wenn der Trocknungsenergieeintrag durch Mikrowellen erhöht wird.

Hierzu werden vier Versuchsreihen in direkter zeitlicher Folge mit nahezu gleichem Feuchtmais durchgeführt: Bei den Versuchen 3.1 und 3.2 betrug der Anfangsfeuchtegehalt $37,5 \%$. Die Versuche 3.3 und 3.4 wurden etwa 10 bis 16 Stunden später durchgeführt, hier 
betrug der gemessene Anfangsfeuchtegehalt 37,0 \%. Die Masse der Feuchtmaisproben betrug jeweils $25000 \mathrm{~g}$. Proben aus diesen Versuchen sind in der CT-Anlage geröntgt worden. Um die Wirkung von Trocknungsenergie auf diesen Körnermais insgesamt einordnen zu können, sind ergänzend CT-Untersuchungen an Feuchtmais und raumluftgetrocknetem Mais durchgeführt worden.

Versuch 3.1: Reine Warmlufttrocknung mit praxistypischen Trocknungsparametern hinsichtlich Warmlufttemperatur und Luftdurchsatz (siehe auch Versuch 2.3.2)

Versuch 3.2: Warmlufttrocknung + Mikrowellenapplikation. Das Hinzuschalten der Mikrowellenenergie erfolgt nach der thermodynamischen Überlegung, den Abluftfeuchtegehalt als Indikator sinkender Trocknungsgeschwindigkeit $\mathrm{zu}$ verwenden. Das Einschalten der Mikrowelle erfolgt nach einem Unterschreiten des Abluftfeuchtegehaltes unter $75 \%$ relativer Feuchte.

Versuch 3.3: Warmlufttrocknung + Mikrowellenapplikation. Hierbei erfolgt bereits ein frühes paralleles Hinzuschalten der Mikrowellenenergie zur Konvektionstrocknung mit zunächst geringer Sendeleistung mit dem Ziel, einen permanent hohen Dampfdruck im Trockengut zu erreichen. Die Mikrowellenapplikation wird im Verlauf des Versuches unterbrochen und nach einem Unterschreiten des Abluftfeuchtegehaltes von $75 \%$ rel. Feuchte erneut zugeschaltet.

Versuch 3.4: Warmlufttrocknung + permanenter Mikrowelleneinsatz. Hier erfolgt die Mikrowellenapplikation mit maximaler Sendeleistung von $1200 \mathrm{~W}_{\mathrm{HF}}$ ergänzend zur Warmlufttrocknung bereits zu Beginn des Versuches. Ziel ist eine möglichst kurze Trocknungszeit unter Inkaufnahme einer deutlich steigenden Korntemperatur zum Trocknungsende.

Der Chargenmischertrockner ist zur Durchführung der Versuche auf dem Betriebsgelände des Pflanzenzuchtunternehmens STRUBE-DIECKMANN im Nebenraum der Röntgenanlage in Söllingen installiert worden, um Körnermaisproben der Sorte Sileno während und nach der Trocknung unmittelbar anschließend zu röntgen. Das Trocknen des Versuches 1 erfolgte 16 Stunden nach dem Dreschen, das Trocknen des Versuches 3.4 erfolgte 34 Stunden nach dem Dreschen des Körnermaises. Der Mais wurde bei einer Außentemperatur von $4{ }^{\circ} \mathrm{C}$ gelagert. Das entsprach auch etwa der Korntemperatur nach dem Dreschen. Witterungsbedingt war der stehende Bestand bis zur Ernte frostfrei.

Basierend auf die in den Trockner mit einem Anfangsfeuchtegehalt von 37,5\% eingefüllte Körnermaismasse von $25 \mathrm{~kg}$ ist entsprechend der Duval'schen Formel 


$$
\mathrm{m}_{\mathrm{H} 2 \mathrm{O}}=\mathrm{m}_{\text {Frischmasse }} \cdot \frac{\mathrm{F}_{\mathrm{A}}-\mathrm{F}_{\mathrm{E}}}{100-\mathrm{F}_{\mathrm{E}}}
$$

$\mathrm{m}_{2} \mathrm{O}=$ Masse Wasser $\quad[\mathrm{kg}]$

$\mathrm{m}_{\text {Frischmasse }}=$ Masse Feuchtmais $\quad[\mathrm{kg}]$

$\mathrm{F}_{\mathrm{A}}=$ Anfangsfeuchtegehalt $\quad[\%]$

$\mathrm{F}_{\mathrm{E}}=$ Endfeuchtegehalt $\quad[\%]$

ermittelt worden, bei welchem Nettogewicht eine Produktfeuchte von etwa
a) $25,0 \%$
b) $17,0 \%$
c) $14,0 \%$ (gleichzeitige Beendigung der Trocknung)

vorliegt. Daraus leitet sich die Probenbezeichnung 3.1a, 3.1b $\left(3.1 \mathrm{c}^{1}\right), 3.2 \mathrm{a}, 3.2 \mathrm{~b}, 3.2 \mathrm{c}$ für die Versuche 3.1 bis $3.4 \mathrm{ab}$. Weil der Chargenmischertrockner auf einer Waage aufgesetzt ist (Auflösung: 0,050 kg), ist eine zeitlich hierauf ausgerichtete Ermittlung der Proben gut möglich. Von jeder Probe wurden drei Feuchtemessungen mit einem geeichten Schnellfeuchtebestimmer (Pfeuffer HE 90) durchgeführt und der Durchschnittswert gebildet.

Einmal aus dem Trockner entnommene Proben wurden nicht wieder in den Trocknungsprozess zurückgeführt. Folglich kommen für die CT-Aufnahmen jeweils neue Körner zum Einsatz.

Während der Durchführung der Trocknungsversuche sind aus Versuch 3.1 (Warmlufttrocknung) zwei Körnermaisproben zu je 200 g entnommen worden. Aus den Versuchen 3.2 bis 3.4 sind jeweils drei Körnermaisproben von ebenfalls je $200 \mathrm{~g}$ entnommen worden. Die Abstände zwischen den Probeentnahmen orientierten sich an dem Gewichtsverlust durch die Trocknung. Zusätzlich zu den unterschiedlichen Trocknungsdurchgängen ist eine Standarduntersuchung von erntefeuchtem Mais und raumluftgetrocknetem Körnermais erfolgt. Diese Proben sind zwei Wochen später geröntgt worden. Insgesamt ergeben sich so 13 unterschiedliche Probengattungen.

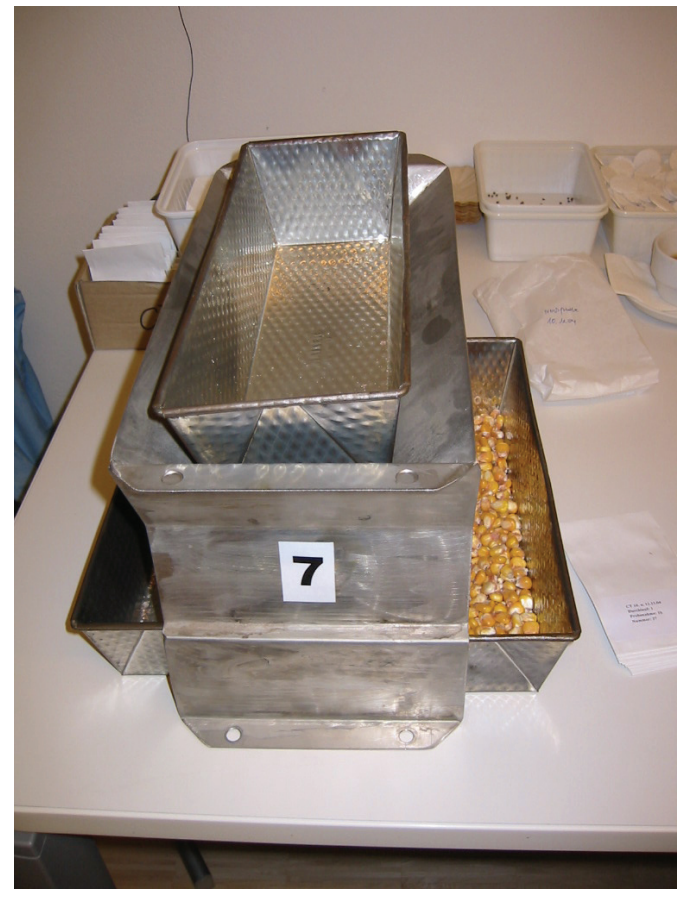

Bild 3.10: Mehrschlitz-Probenteiler 
Die jeweiligen $200 \mathrm{~g}$ Proben sind mit einem Mehrschlitz-Probenteiler (Bild 3.10) geteilt worden. So sind aus jeder Probe mit etwa 800 Körnern im Ergebnis 48 Körner differenziert worden, die zu jeweils 12 Körnern in vier Durchgängen geröntgt wurden. Jedes einzelne Korn ist entsprechend seiner Einbettung in der Aufnahmeapparatur nummeriert worden und steht in einer Archivierungsregistratur weiterhin zerstörungsfrei zur Verfügung.

\subsubsection{Mikro-Computertomographie}

\subsection{Computertomographie}

Nach der Probenteilung erfolgte eine einheitliche Homogenisierungszeit von ca. 60 Minuten an Umgebungsluft $\left(20{ }^{\circ} \mathrm{C}, 65 \%\right.$ rel. Luftfeuchte), bevor die Proben geröntgt wurden. Hierzu wurden jeweils 12 Körner einer Probe in den aus Styropor bestehenden Probenträger eingeklemmt. Dieser wurde anschließend auf dem Rotationsteller in der Röntgenkammer befestigt. Nach dem rechnergeführten Starten der Röntgenanlage rotiert der Probenträger nach jeweils 2 Sekunden um $1^{\circ}$ um seine vertikale Achse, so dass für jeweils 12 Körner eine Aufnahmedauer von 12 Minuten erforderlich ist. Einschließlich der Nebenzeiten ergeben sich ca. 15 Minuten für jeden Durchgang.

\subsection{Segmentierung}

Das mit der Röntgenanlage verknüpfte Rechnersystem trennt entsprechend einer definierbaren Kontrastskala Graustufen der Röntgenabbildung in einer Durchleuchtungsebene in schwarze und weiße Felder. Hierdurch wird eine vergleichsweise scharfkantige Ebenenabbildung erreicht. Durch Vorversuche im Hause Strube-Dieckmann sind die für eine Bewertung entsprechend den geometrischen Eigenarten des Maiskorns passenden Kontrastparameter ermittelt worden. Die Segmentierung ist für die rechnergestützte Binärbilderzeugung erforderlich. 


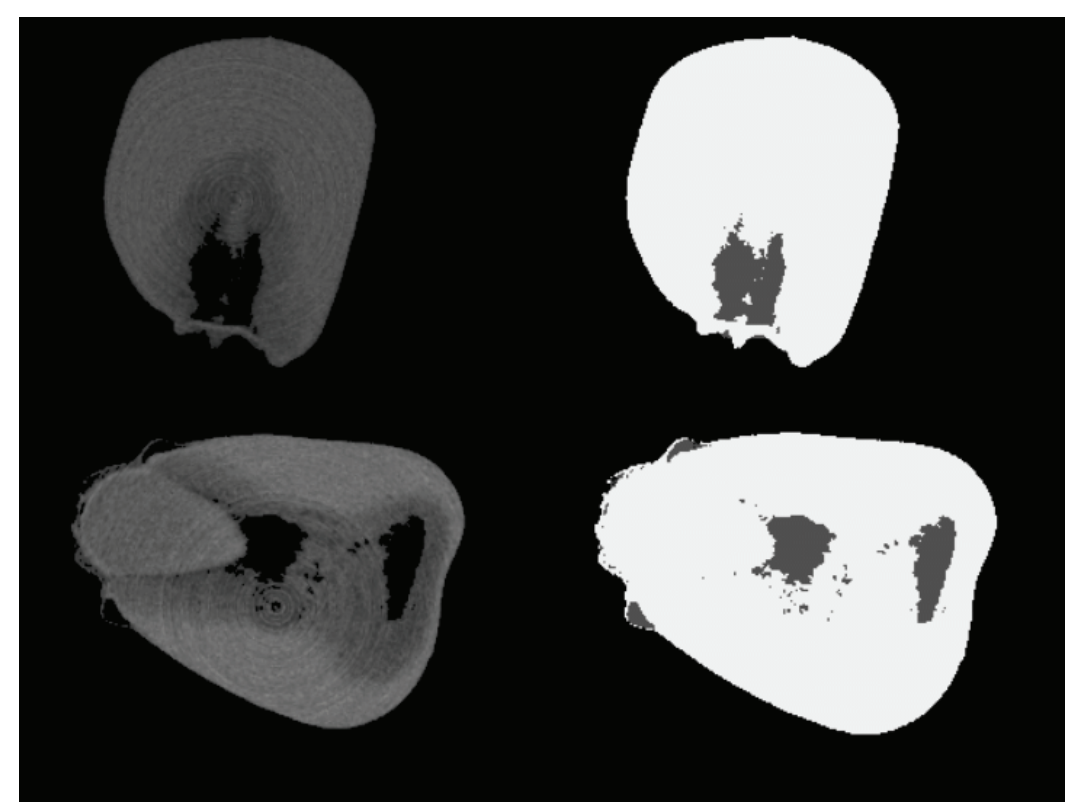

Bild 3.11: Röntgenaufnahme (linke Abbildungen) eines Maiskorns in einer Schnittebene, rechtsseitig das dazugehörige segmentierte Bild.

Die CT-Aufnahme aus Bild 3.11 zeigt mit $125{ }^{\circ} \mathrm{C}$ Warmluft getrockneten Körnermais, rechtsseitig die Segmentierung. Auf dieser Basis erfolgt die weitere rechnerische Verwertung. Durch Überlagerung ergeben sich dann im Volumenmodell wieder Grauschattierungen (Tiefenwirkung).

\subsection{Bildgenerierung}

Für eine dreidimensionale Betrachtung der Maisproben werden aus den drei Schnittebenen (xy; x-z; y-z) verwertbare Bilder konfiguriert. Diese erlauben Aussagen zur Produktgeometrie, Volumenänderungen und Verhältnisänderungen von Fest- zu Hohlkörperanteil und insbesondere auch deren räumliche Ausbreitung und Gestaltung. Weiterführend lassen sich so auch typische Ausbreitungsrichtungen ermitteln. Auf diese Weise lassen sich Erkenntnisse herleiten, welche Kraftrichtungen durch den Aufbau von Dampfdruck während der Trocknung von Maiskörnern entstehen. Die in dieser Ausarbeitung abgebildeten Vollkörperbilder der Körnermaisproben entsprechen hinsichtlich der Volumenverhältnisse (Volumen insgesamt und Verhältnis von Fest- zu Hohlkörper) am nächsten dem Durchschnittswert einer Probe. Dargestellt sind jeweils die Endtrocknungszustände. 


\subsubsection{Sorptionsverhalten}

Nach der Durchführung der Trocknungsversuche und der Erstellung der unmittelbar sich hieran anschließenden computertomographischen Aufnahmen sind die getrockneten Körnermaisproben (jeweils ca. $18000 \mathrm{~g}$ ) der vier Versuche zunächst für 150 Tage unter lagertypischen Bedingungen bei $15{ }^{\circ} \mathrm{C}$ und $65 \%$ rel. Raumluftfeuchte gelagert worden.

Die Aufnahme von Adsorptions- und Desorptionsisothermen gilt als ein wissenschaftlich gesichertes Verfahren zur Bewertung von Befeuchtungs- und Entfeuchtungsvorgängen, gleichwohl ebenso zur Ermittlung von Gleichgewichtszuständen. Entsprechend der vier energetisch unterschiedlich durchgeführten Versuche sollen die Erkenntnisse der Mikroröntgentechnik dem Sorptions- bzw. Desorptionsverhalten der Körnermaisproben gegenüber gestellt werden.

Zur Vorbereitung werden aus den verschiedenen Trocknungsversuchen 3.1 bis 3.4 zunächst je 5 Proben mit einer Masse von etwa $30 \mathrm{~g}$ entnommen, wovon jeweils in zwei der Probenschalen ganzkörnige Maiskörner (Probenzusatzbezeichnung a) manuell sortiert werden und in zwei weitere Probeschalen gebrochene oder geschlitzte Körner gefüllt werden (Probenzusatzbezeichnung b). Diese Körner sind nicht zusätzlich zerstört worden, sondern entstammen dem jeweiligen Trocknungsgut. So werden mögliche Unschärfen aufgrund anderer Diffusionensgeschwindigkeiten durch eine zerstörte Maiskornschale offensichtlich, die bei einer Gemengeprobe nicht zur ermitteln sind. Um die Kornfeuchtegehalte nach Erreichen der Beharrungszustände im Klimaschrank festzustellen, werden vier weitere Kontrollproben (3.1k - 3.4k) entnommen und der Produktfeuchtegehalt nach der Trockenschrankmethode bestimmt. Insgesamt ergeben sich somit insgesamt 4 × $5=20$ Einzelproben. Die aus Drahtgewebe bestehenden Probeschalen ermöglichen einen ungehinderten Feuchteaustausch der einzelnen Maiskörner mit der umgebenden Atmosphäre. Nachfolgendes Schaubild verdeutlicht die Vorgehensweise beispielhaft für die Aufteilung und Probenbenennung aus Versuch 3.1, wobei derselbe Bezeichnungsschlüssel auch für die Proben aus den Versuchen 3.2 bis 3.4 besteht. 


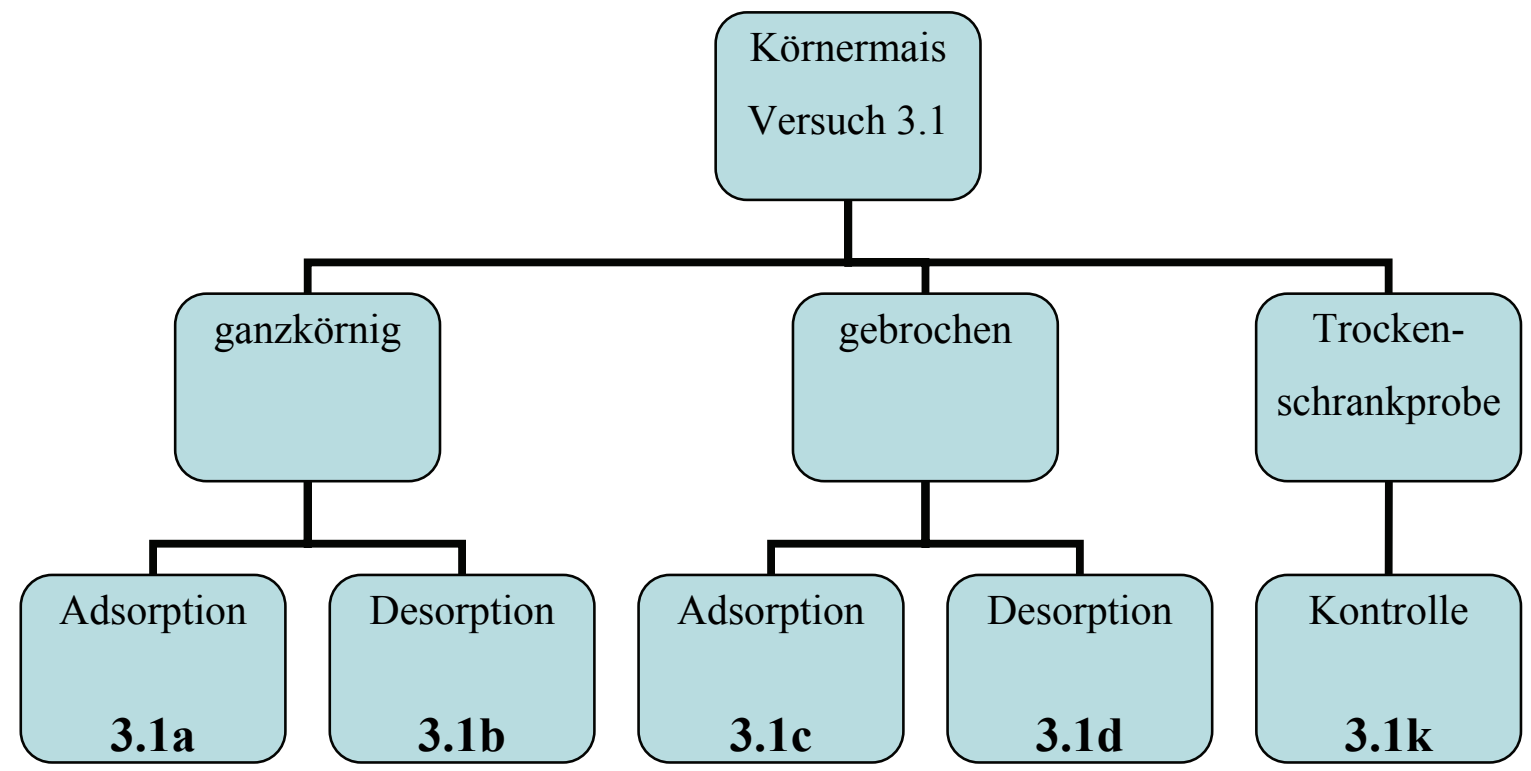

Bild 3.12: Übersicht Probenbenennung der Sorptionsversuche am Beispiel Versuch 3.1

Nach dem Auswiegen der Proben (Auflösung 0,001 g) folgt zunächst ein 48 tägiges Verweilen (1152 Stunden) in einem Klimaschrank (Bild 3.13) mit einem eingestellten Innenluftfeuchtegehalt von $62,0 \%$ bei $30{ }^{\circ} \mathrm{C}$ Lufttemperatur. Entsprechend vorliegender Sorptionsisothermen für Mais sollte dieses einer Gleichgewichtsfeuchte von 12,3\% entsprechen. Es ist aber zu berücksichtigen, dass durch sorten- und standortsignifkante Einflüsse hiervon aber Abweichungen möglich sind (Kersten 2004). Wichtig ist, dass sich alle Proben im Zustand des Feuchtegleichgewichtes befinden, bevor die eigentliche Aufnahme des Adsorptions- und Desorptionsverhaltens geschieht.

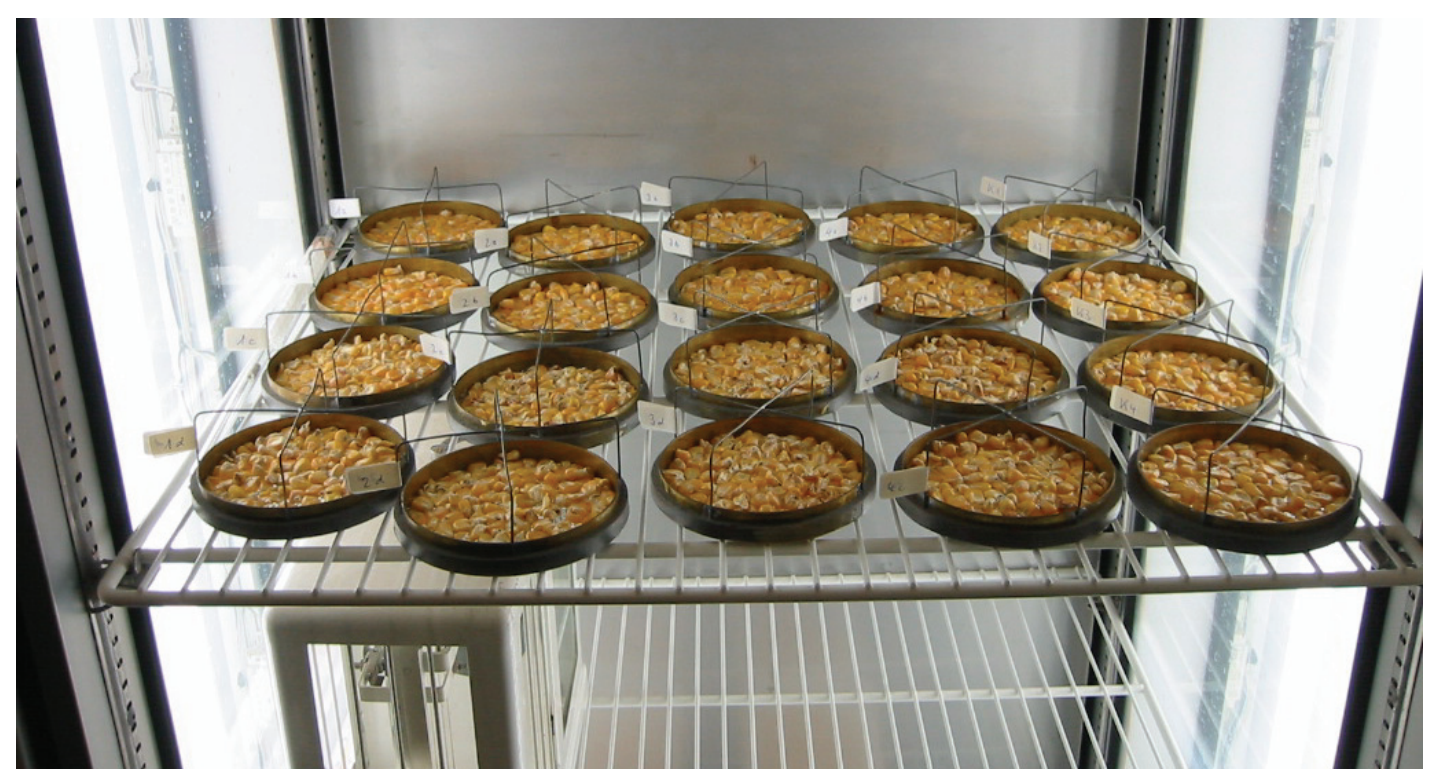

Bild 3.13: Körnermaisproben der Versuche 3.1 bis 3.4 in Probenträgern aus Drahtgewebe, Ansicht in den Klimaschrank. 
Zur Adsorption, also zur Feuchtigkeitsaufnahme des Maises aus der möglichen Umgebung innerhalb des Glasbehälters wird eine übersättigte Amoniumsulfatlösung ((NH4) $\left.)_{2} \mathrm{SO} 4\right)$ verwendet, wobei sich bei einer Beharrungstemperatur von $30{ }^{\circ} \mathrm{C}$ ein relativer Raumfeuchtegehalt von $80 \%$ einstellt. Zur Desorption, also zur Feuchtigkeitsabgabe des Maises über die Zustände des Klimaschrankes hinaus, wird innerhalb des Glasbehälters eine untersättigte Magnesiumchloridlösung $(\mathrm{MgCl} 2)$ benutzt. Hier stellt sich bei einer gleichen Beharrungstemperatur von $30{ }^{\circ} \mathrm{C}$ eine relative Raumfeuchte von $33 \%$ ein. Hierzu werden die einzelnen Drahtgewebeschalen mit dem darin befindlichen Körnermais in geschlossenen Glasbehältern isoliert. In den Glasbehältern befinden sich jeweils nicht gesättigte bzw. übersättigte Salzlösungen, die im Ergebnis zu einer Befeuchtung oder Entfeuchtung der Maisproben führen.

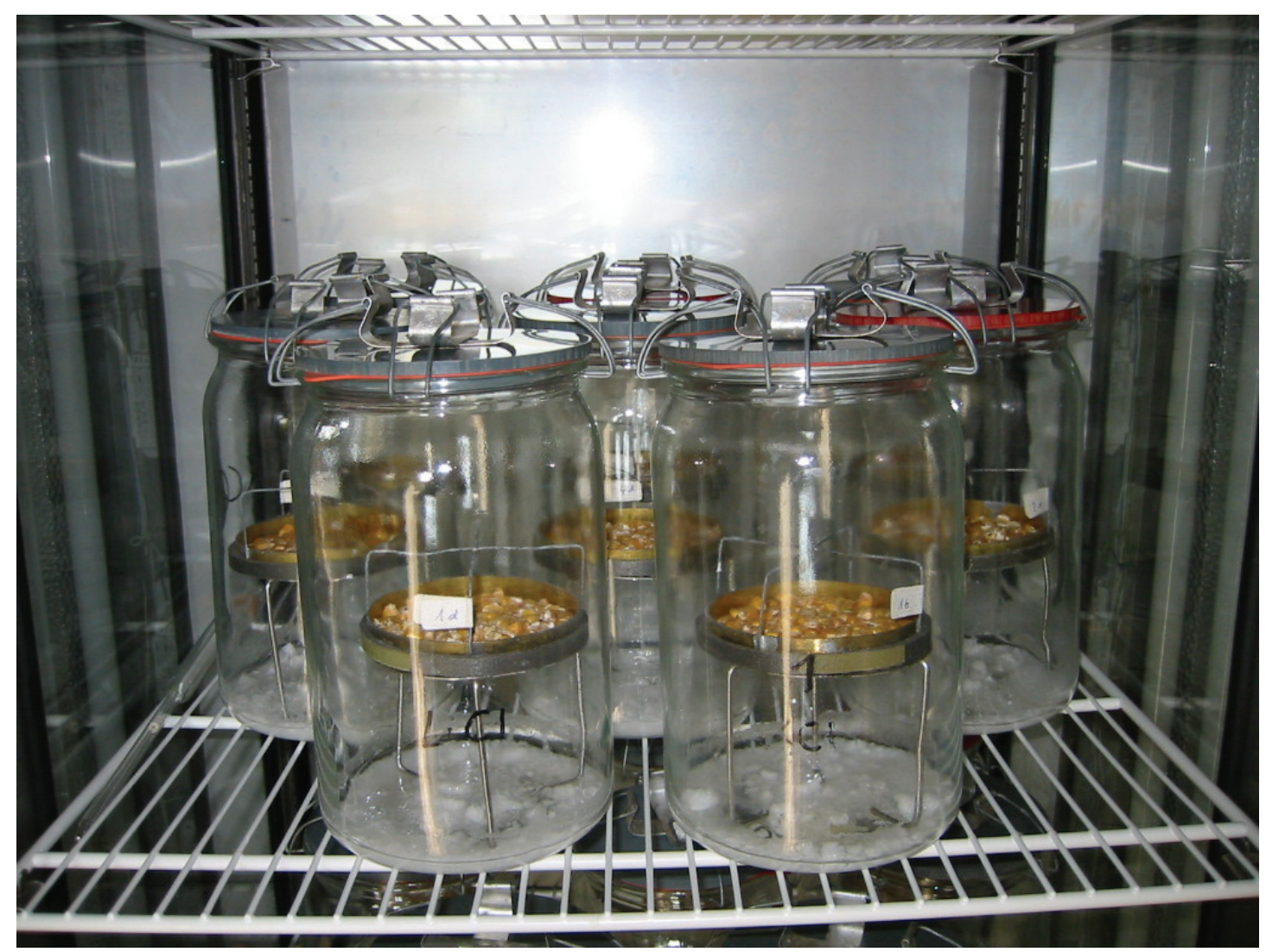

Bild 3.14: Körnermaisproben in Glasbehältern mit Salzlösung. Die Glasbehälter sind im Klimaschrank mit einer Innenraumtemperatur von $30^{\circ} \mathrm{C}$ aufgestellt.

Die Zustandsänderungen der Maisproben wurden über einen Zeitraum von insgesamt 50 Tagen aufgenommen. Danach bildete sich an den Proben in der 80 \%igen Raumluftatmosphäre Schimmel, so dass eine weitere Bewertung ausgeschlossen ist. Für die spätere Bewertung wird daher nur der Zeitraum der ersten 1000 Stunden (ca. 41 Tage) berücksichtigt. 


\subsection{Ergebnisse}

In den Bildern 3.15 bis 3.18 sind die wesentlichen Ergebnisse der Versuche zur Körnermaistrocknung mit Warmluft bzw. Warmluft und Mikrowellenenergie grafisch dargestellt.

\subsubsection{Versuch 1}

Zur besseren Vergleichbarkeit von Versuch 3.1 mit dem Versuch 2.2 wurde zunächst für die ersten $75 \mathrm{~min}$ Trocknungsdauer eine Warmlufttemperatur von $80{ }^{\circ} \mathrm{C}$ eingestellt. Im Unterschied zu Versuch 2.2 betrug der Anfangsfeuchtegehalt nicht $29 \%$ sondern bei diesem Versuch 37,5 \%. Es zeigt sich, dass der Abluftfeuchtegehalt bereits nach der 45. Trocknungsminute kontinuierlich abfällt, wobei die Erhöhung der Warmlufttemperatur nach der 75. Trocknungsminute auf ca. $125{ }^{\circ} \mathrm{C}$ kurzzeitig zu einer Erhöhung der Wasserbeladung der Abluft auf 25,1 $\mathrm{g} \mathrm{m}^{-3}$ führt, um dann bis zum Ende der Trocknung nach 225 Minuten auf 16,0 $\mathrm{g} \mathrm{m}^{-3}$ abzufallen. Während dieses Versuches konnte - ebenso wie bei den Versuchen 3.2 bis 3.4 - die Korntemperatur nicht gemessen werden. In Vorversuchen hat sich aber gezeigt, dass bei dem Versuchstrockner aufgrund des chargenweisen Betriebes die Ablufttemperatur mit der Korntemperatur in Korrelation zu sehen ist.

Um in der Körnermaisschüttung unterschiedliche Trockengutschichtungen zu vermeiden, ist das Trocknungsgut permanent gerührt worden (Ciesler 1986). Zum Ende des Versuches betrug die Ablufttemperatur $52,0^{\circ} \mathrm{C}$ bei einem Kornfeuchtegehalt von 14,2\%. Aus der Anfangsmasse von $25000 \mathrm{~g}$ sind $6400 \mathrm{~g}$ Wasser getrocknet worden, $400 \mathrm{~g}$ sind für die CT Untersuchungen während der Trocknung entnommen worden. Der spezifische Trocknungsenergiebedarf betrug $1,33 \mathrm{kWh} \mathrm{kg}^{-1}$ Wasserentzug. 


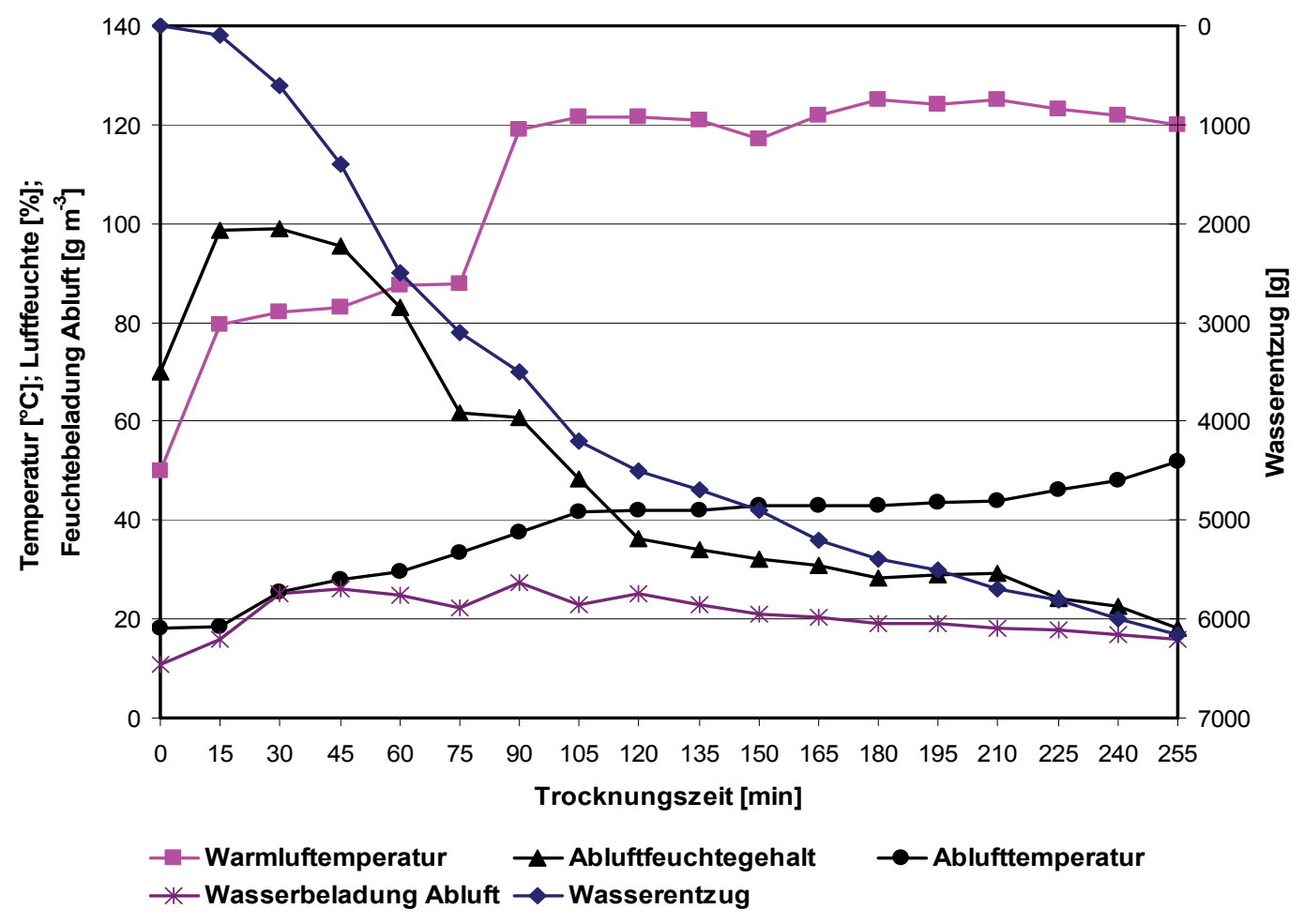

Bild 3.15: Warmlufttrocknung im Chargenmischertrockner, Ergebnisse aus Versuch 3.1

\subsubsection{Versuch 2}

Im Unterschied zum voran stehenden Versuch wurde ergänzend zur Warmlufttrocknung Mikrowellenenergie unterstützend eingesetzt. Als Einschaltkriterium ist der Abluftfeuchtegehalt gewählt worden. Nach Unterschreiten von $75 \%$ rel. Feuchte wurde die Mikrowellenapplikation mit $960 \mathrm{~W}$ Sendeleistung für 45 Minuten eingeschaltet. Die Wasserbeladung der Abluft hat daraufhin ein Maximum von 36,1 $\mathrm{g} \mathrm{H}_{2} \mathrm{O} \mathrm{m}^{-3}$ erreicht. Zum Ende der Trocknung ist der Abluftfeuchtegehalt auf 16,5\% abgefallen. Der erforderliche Wasserentzug durch Trocknung von $6600 \mathrm{~g}$ ist bereits nach der 225. Trocknungsminute erreicht worden, die Ablufttemperatur erreichte zum Ende der Trocknung 56,3 ${ }^{\circ} \mathrm{C}$. Bei einem Energieeinsatz von $8,70 \mathrm{kWh}$ für Warmluft und einem Energieeinsatz für die Mikrowellenapplikation von 1,75 kWh ergab sich zum Trocknen eine eingesetzte Energie von $10,45 \mathrm{kWh}$. Der spezifische Trocknungsenergiebedarf betrug somit 1,58 $\mathrm{kWh} \mathrm{kg}^{-1}$ Wasserentzug. Im Verhältnis zum Gesamtenergieeinsatz beträgt der prozentuale Anteil Mikrowellenenergie bei diesem Versuch 16,74 \%. 


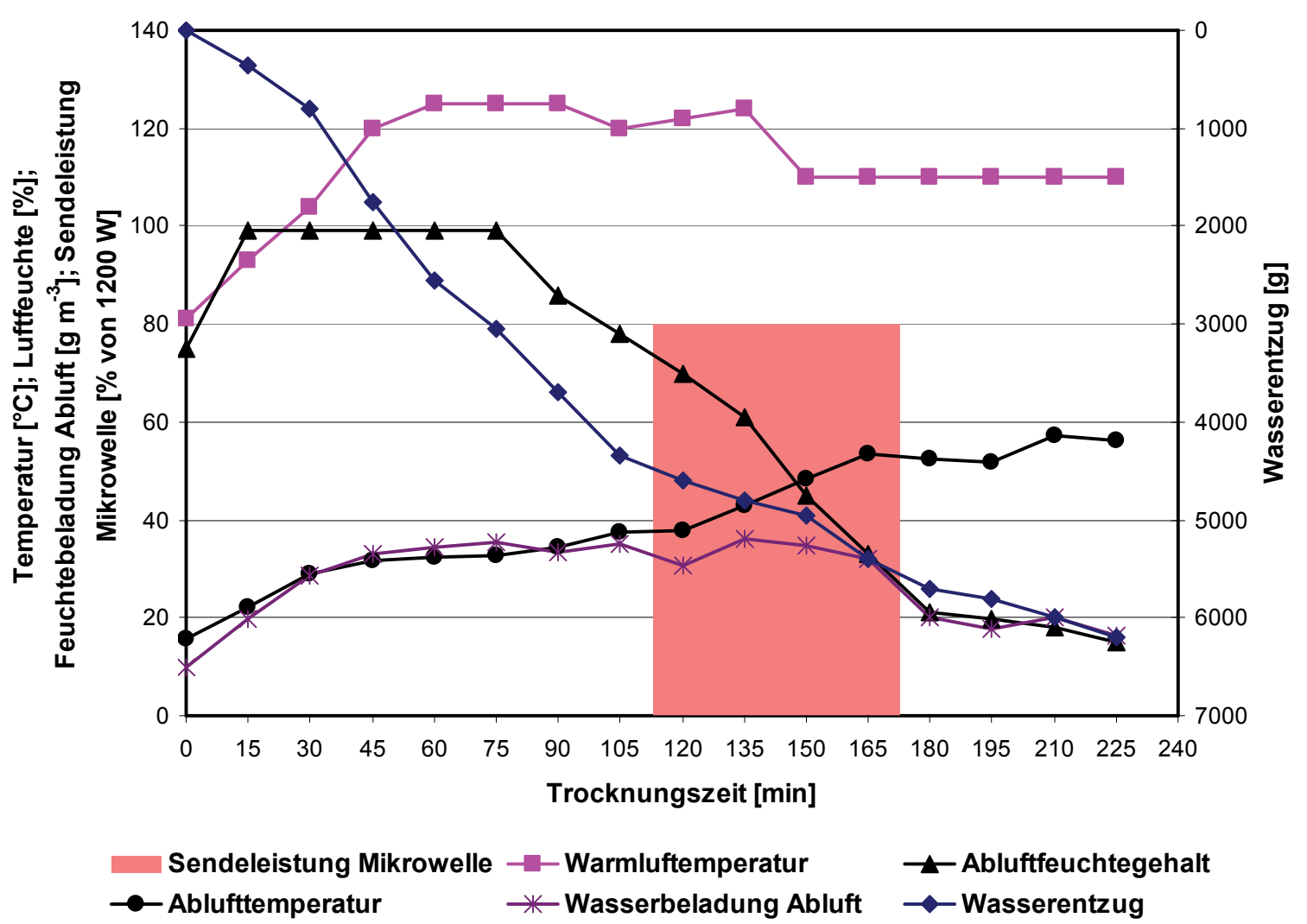

Bild 3.16: Mikrowellenunterstützte Warmlufttrocknung im Chargenmischertrockner, Ergebnisse aus Versuch 3.2

\subsubsection{Versuch 3}

Bei diesem Versuch wurde im Unterschied zu Versuch 3.2 die Mikrowellenapplikation in zwei Aktionsfenstern zugeschaltet. Hierbei stand die Überlegung im Vordergrund, Mikrowellenenergie im Korninnern bereits bei noch vorhandener höherer Gutfeuchte wirken zu lassen, um so den Feuchteentzug in der ersten Trocknungsstunde bei einer gewählten Warmlufttemperatur von ca. $80{ }^{\circ} \mathrm{C}$ zu unterstützen. Obwohl noch ein ausreichend hoher Abluftfeuchtegehalt nahe der Sättigung der Abluft vorlag, wurde trotzdem schon Mikrowellenenergie mit einer Sendeleistung von $600 \mathrm{~W}$ bereits nach ca. 20 Minuten Trocknungsdauer für zunächst 45 Minuten appliziert. Nach einem signifikanten Abfallen des Abluftsättigungsgehaltes auf ca. $50 \quad \%$ nach 75 Trocknungsminuten wurde die Warmlufttemperatur auf ca. $120{ }^{\circ} \mathrm{C}$ erhöht. Als Einschaltkriterium des zweiten Aktionsfensters für die Mikrowellenapplikation wurde das Unterschreiten der Wasserbeladung der Abluft unter $25 \mathrm{~g} \mathrm{~m}^{-3}$ gewählt, wobei die Mikrowellenapplikation dann mit einer Sendeleistung von $1200 \mathrm{~W}$ für 30 Minuten zugeschaltet worden ist. 
Der erforderliche Wasserentzug durch Trocknung von $6600 \mathrm{~g}$ ist bereits nach 180 Trocknungsminuten erreicht worden, die Ablufttemperatur erreichte zum Ende der Trocknung $63,7^{\circ} \mathrm{C}$. Bei einem Energieeinsatz von 7,95 kWh für Warmluft und einem Energieeinsatz für die Mikrowellenapplikation von 2,55 kWh ergab sich zum Trocknen eine eingesetzte Energie von 10,50 kWh. Der spezifische Trocknungsenergiebedarf betrug somit $1,59 \mathrm{kWh} \mathrm{\textrm {kg } ^ { - 1 }}$ Wasserentzug. Im Verhältnis zum Gesamtenergieeinsatz beträgt der prozentuale Anteil Mikrowellenenergie bei diesem Versuch 24,28 \%.

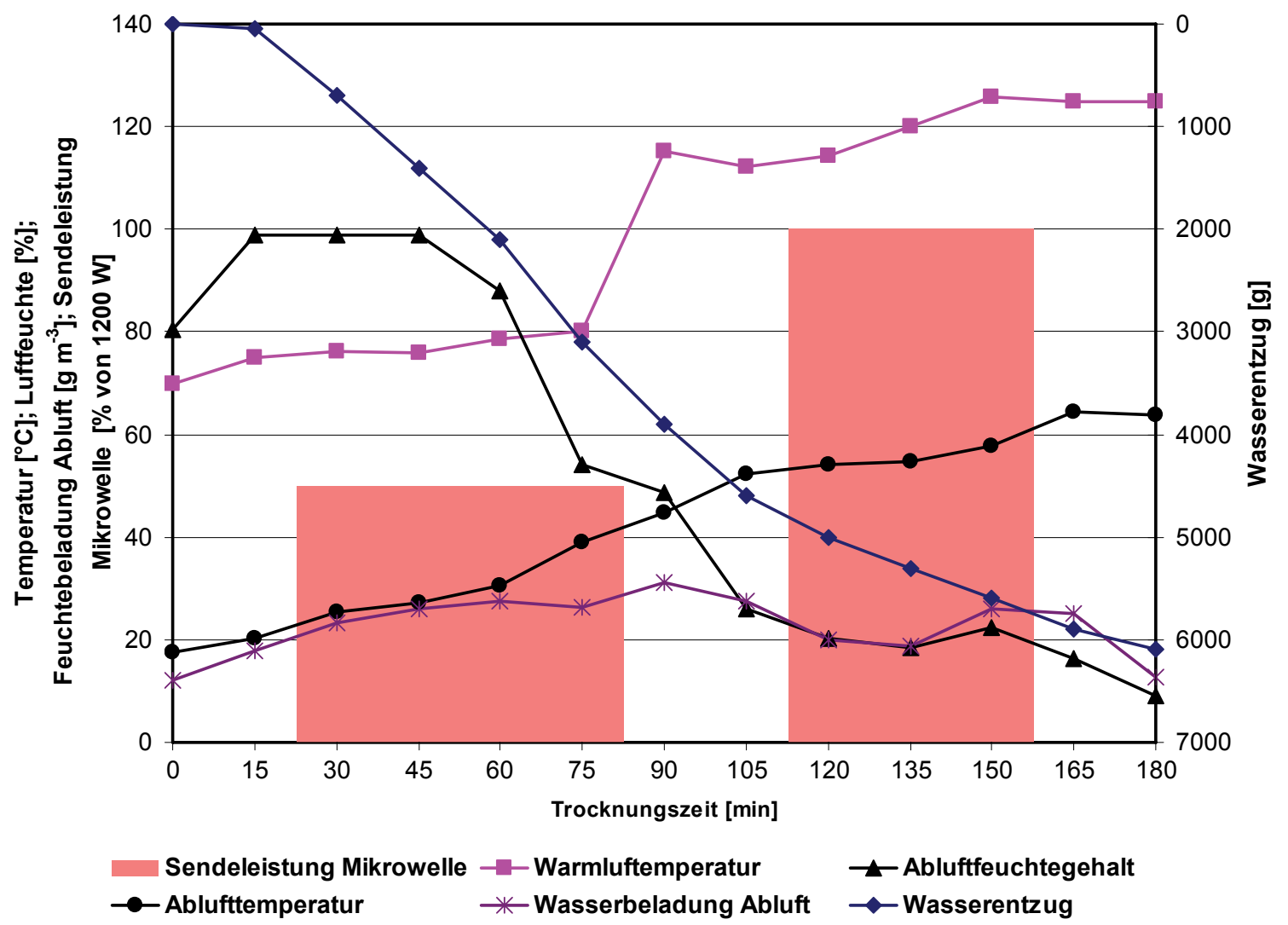

Bild 3.17: Mikrowellenunterstützte Warmlufttrocknung im Chargenmischertrockner, Ergebnisse aus Versuch 3.3

\subsubsection{Versuch 4}

Bei den Versuchen 3.1 bis 3.3 orientierte sich die Einstellung der gewählten Parameter hinsichtlich der Warmlufttemperatur, Prozessluftmenge und Mikrowellenenergie an den Überlegungen, in unterschiedlichen Kombinationen möglichst wenig Trocknungsenergie aufwenden $\mathrm{zu}$ müssen und auch kurze Trocknungszeiten anzustreben. Die Zielsetzung in Versuch 3.4 ist es, von Anfang an mit einer hohen Warmlufttemperatur von ca. $130{ }^{\circ} \mathrm{C}$ und einer Mikrowellenapplikation mit einer Sendeleistung von 1200 W eine möglichst kurze Trocknungszeit zu erreichen. Die Mikrowellenapplikation ist nach 100 Trocknungsminuten 
abgeschaltet worden, nach der 135. Trocknungsminute waren $6700 \mathrm{~g}$ Wasser entzogen. Durch Messungen wurde ein Kornfeuchtegehalt von 14,1\% festgestellt. Die Ablufttemperatur betrug zum Ende der Trocknung 65,3 ${ }^{\circ} \mathrm{C}$. Bei einem Energieeinsatz von $6,9 \mathrm{kWh}$ für Warmluft und einem Energieeinsatz für die Mikrowellenapplikation von 4,45 kWh ergab sich zum Trocknen eine eingesetzte Energie von 11,35 kWh. Der spezifische Trocknungsenergiebedarf betrug somit 1,69 $\mathrm{kWh} \mathrm{kg}^{-1}$ Wasserentzug. Im Verhältnis zum Gesamtenergieeinsatz beträgt der prozentuale Anteil Mikrowellenenergie bei diesem Versuch 39,20 \%.

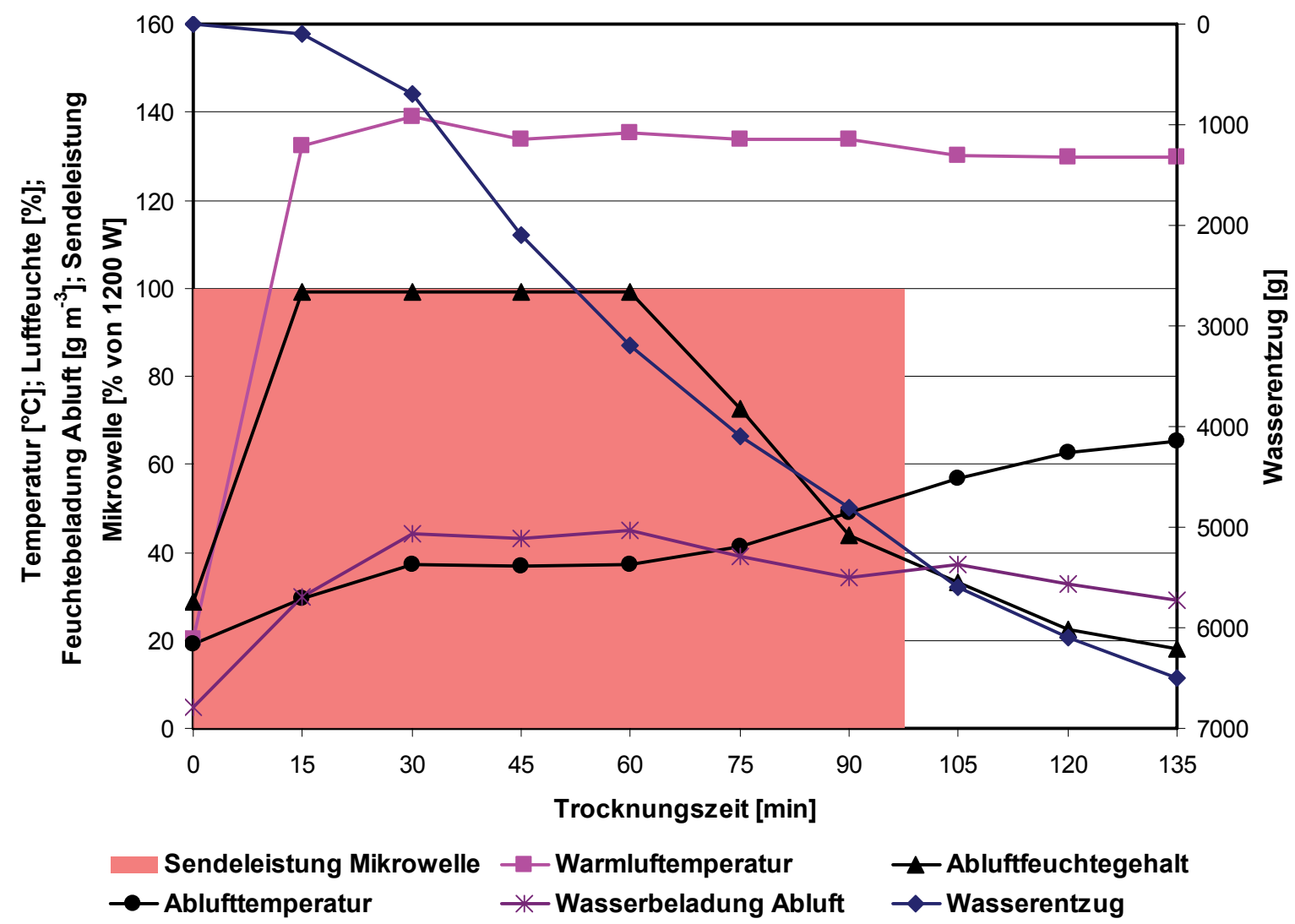

Bild 3.18: Mikrowellenunterstützte Warmlufttrocknung im Chargenmischertrockner, Ergebnisse aus Versuch 3.4

\subsubsection{Computertomographie}

\subsubsection{Summenauswertung der Volumenverhältnisse}

Tabelle 3.2 zeigt in einer Durchschnittswertauflistung das Gesamtvolumen, den Volumenanteil Festkörper und den Volumenanteil Hohlraum sowie das prozentuale Hohlraumvolumen aller untersuchten Maiskörner an. Die Mittelwerte ergeben sich aus den Ergebnissen der jeweils 48 je Probe untersuchten Körner. Der Kornfeuchtegehalt ist in der rechten Spalte aufgeführt. 
Tabelle 3.2: Summenauswertung; Volumenverhältnisse der Maisproben Versuch 3.1 bis 3.4, feuchter und raumluftgetrockneter Körnermais

\begin{tabular}{cccccc}
\hline Versuch & $\begin{array}{c}\text { Gesamt Vol. } \\
{\left[\mathrm{mm}^{3}\right]}\end{array}$ & $\begin{array}{c}\text { Vol. Festkörper } \\
{\left[\mathrm{mm}^{3}\right]}\end{array}$ & $\begin{array}{c}\text { Vol. Hohlraum } \\
{\left[\mathrm{mm}^{3}\right]}\end{array}$ & $\begin{array}{c}\text { Hohlraum } \\
{[\%]}\end{array}$ & $\begin{array}{c}\text { Feuchtegehalt } \\
{[\%]}\end{array}$ \\
\hline $3.1 \mathrm{a}$ & 266,18 & 248,84 & 17,33 & 6,32 & 19,3 \\
$3.1 \mathrm{~b}$ & 284,10 & 259,31 & 24,80 & 8,89 & 14,2 \\
$3.2 \mathrm{a}$ & 300,14 & 288,35 & 11,79 & 3,87 & 25,5 \\
$3.2 \mathrm{~b}$ & 289,53 & 265,62 & 23,90 & 8,39 & 17,0 \\
$3.2 \mathrm{c}$ & 291,11 & 268,88 & 22,22 & 7,74 & 14,2 \\
$3.3 \mathrm{a}$ & 308,80 & 296,29 & 12,50 & 4,00 & 23,6 \\
$3.3 \mathrm{~b}$ & 299,80 & 281,50 & 18,30 & 6,05 & 17,9 \\
$3.3 \mathrm{c}$ & 282,28 & 258,08 & 24,20 & 8,56 & 14,1 \\
$3.4 \mathrm{a}$ & 300,57 & 287,91 & 12,65 & 4,26 & 23,8 \\
$3.4 \mathrm{~b}$ & 294,65 & 276,36 & 18,28 & 6,41 & 18,7 \\
$3.4 \mathrm{c}$ & 279,97 & 251,07 & 28,90 & 10,30 & 14,1 \\
feucht & 335,82 & 326,51 & 9,32 & 2,89 & 37,5 \\
raumluftgetrocknet & 264,55 & 252,77 & 11,78 & 4,45 & 14,8 \\
\hline
\end{tabular}

\subsubsection{Computertomographische Aufnahmen}

Die nachfolgende Übersicht dreidimensionaler Maiskörner dient zur Verdeutlichung der durch Trocknung im Korninnern hervorgerufenen Strukturänderungen. Die Bilder sind aus realen Maiskörnern regeneriert, wobei aber nur die Endtrocknungszustände dargestellt sind. Hierbei sind solche Maiskörner ausgewählt worden, die hinsichtlich der geometrischen Verhältnisse dem Durchschnittswert aller Körner einer jeden Probe aus Tabelle 3.2 am ehesten entsprechen. Jeweils links ist das vollständige Korn abgebildet, die rechte Ansicht zeigt das Korn im Schnitt.
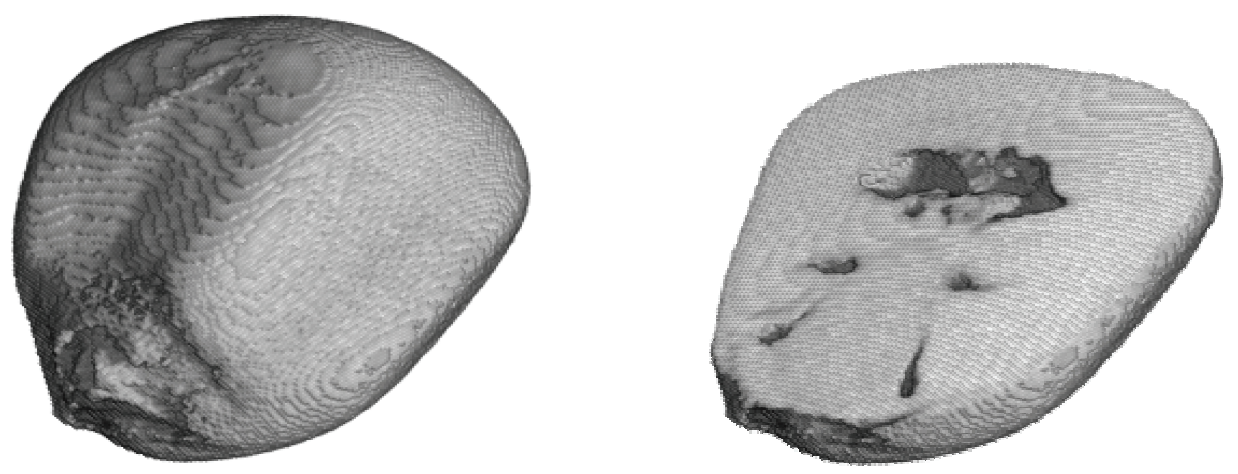

Bild 3.19: CT-Aufnahme, dreidimensional, Kontrolle feuchtes Maiskorn $37,5 \%$ Feuchtegehalt, Hohlraumanteil 2,89\% 

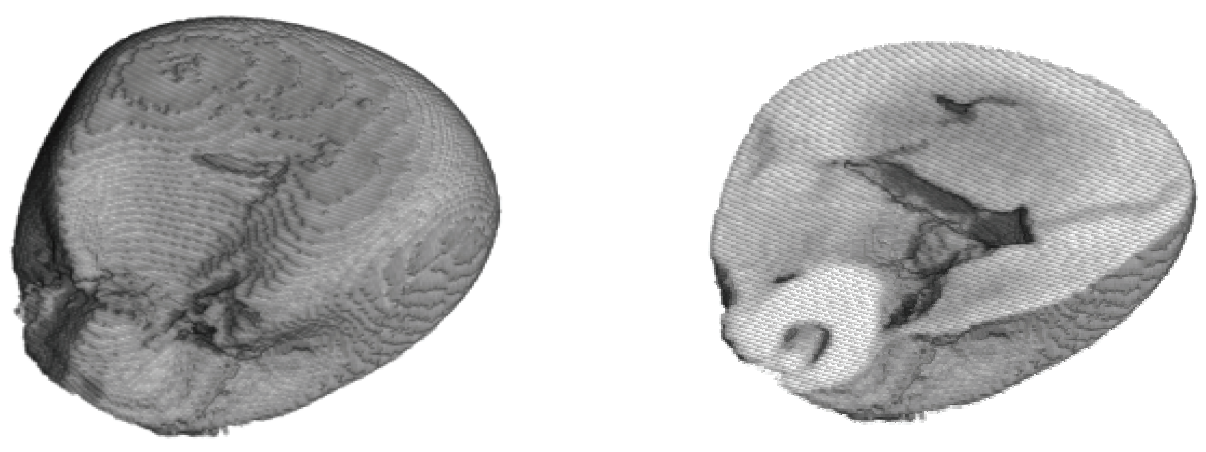

Bild 3.20: CT-Aufnahme, dreidimensional, Kontrolle Raumlufttrocknung, 14,8 \% Feuchtegehalt, Hohlraumanteil 4,45\%
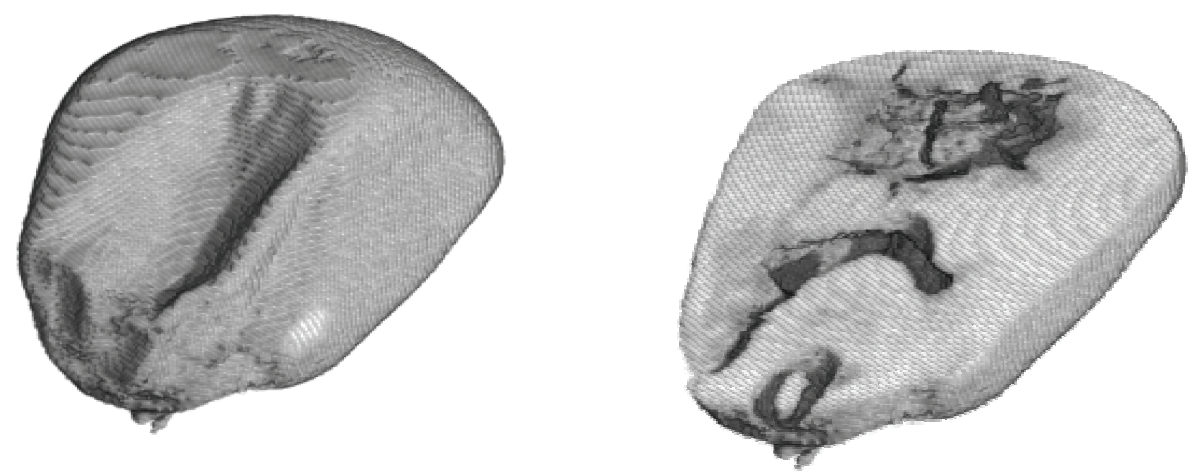

Bild 3.21: CT-Aufnahme, dreidimensional, Versuch 3.1, Warmlufttrocknung 14,2 \% Feuchtegehalt, Hohlraumanteil 8,89\%
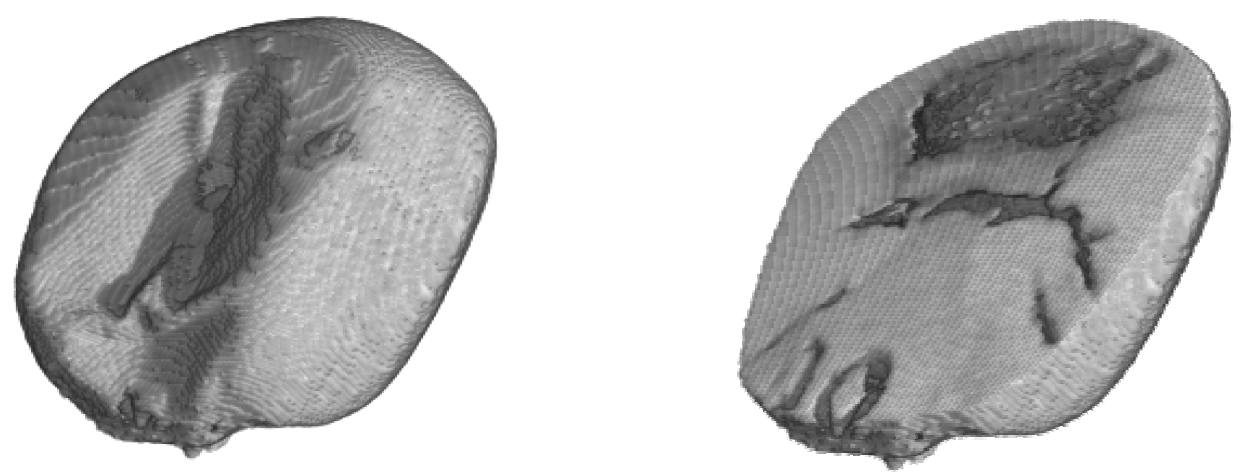

Bild 3.22: CT-Aufnahme, dreidimensional, Versuch 3.2, Warmlufttrocknung + MW nach thermodynamischen Überlegungen, 14,2 \% Feuchtegehalt, Hohlraumanteil 7,74 \% 

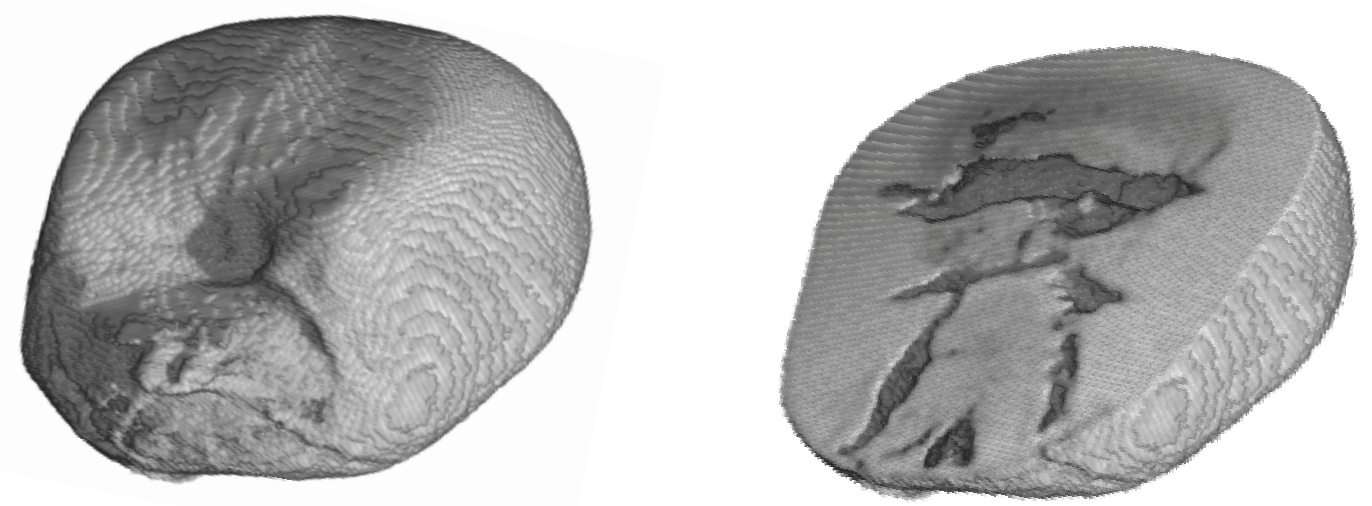

Bild 3.23: CT-Aufnahme, dreidimensional, Versuch 3.3, Warmlufttrocknung + Mikrowelle unterbrochen und mit reduzierter Sendeleistung, 14,1 \% Feuchtegehalt, Hohlraumanteil 8,56\%
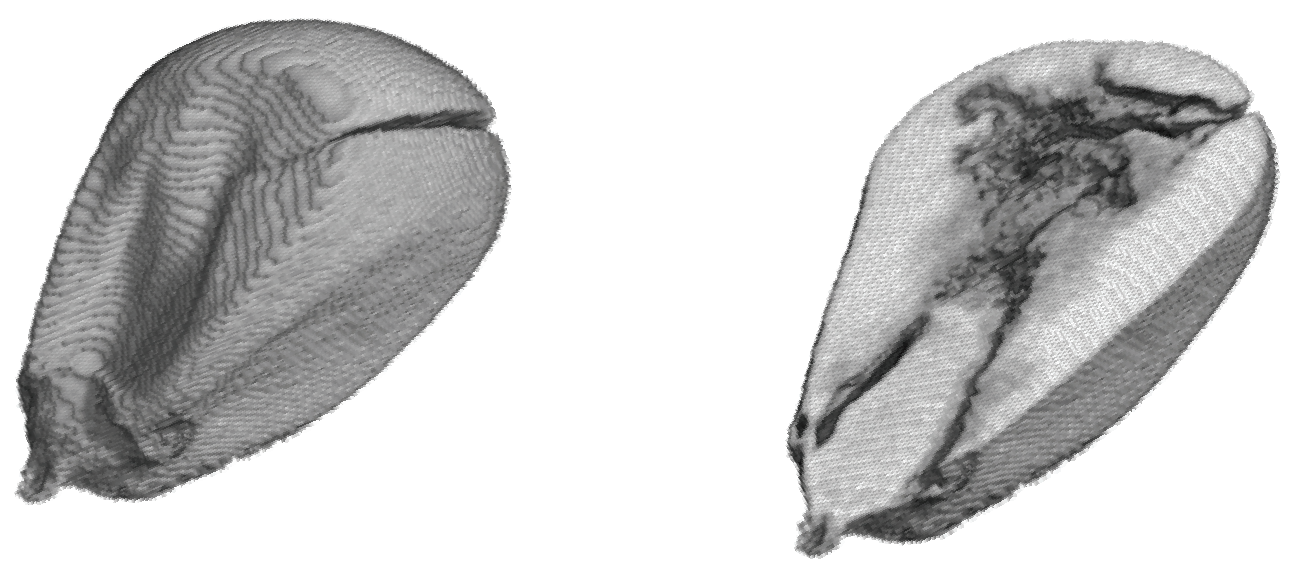

Bild 3.24: CT-Aufnahme, dreidimensional, Versuch 3.4, Warmlufttrocknung + Mikrowelle permanent, 14,1 \% Feuchtegehalt, Hohlraumanteil 10,30\%

\subsubsection{Darstellung des Sorptionsverhaltens}

Der Ermittlung des Sorptionsverhaltens gingen zwei Lagerungszustände voraus, um die Versuche in gesättigen Salzlösungen von einem stationären Gleichgewichtszustand aus beginnen zu können.

Zunächst wurden die getrockneten Maiskörner entsprechend der durch die Trocknungsversuche 3.1 bis 3.4 vorgegebene Unterteilung für die Dauer von 150 Tagen unter lagertypischen Umgebungsluftzuständen bei einer durchschnittlichen Temperatur von $15{ }^{\circ} \mathrm{C}$ und $60 \%$ relativer Luftfeuchte gelagert. Nach der Ernte und Konservierung laufen die für das Korn 
notwendigen Stoffwechselprozesse auch während der Lagerung weiter ab (Morcos 1986). Entscheidend werden diese von den Lagerbedingungen, insbesondere den Luftzuständen, beeinflusst. Aus der literaturbekannten Sorptionsisotherme ist unter diesen Lagerkonditionen eine sich einstellende Gleichgewichtsfeuchte von 13,0 \% zu erwarten (Goldsaat 1988). In Tabelle 3.3 sind die tatsächlich gemessenen Kornfeuchtegehalte aufgeführt, die mit einem geeichten Schnellfeuchtebestimmer (Pfeuffer HE 90) und nach der Trockenschrankmethode ermittelt worden sind, bevor die Proben nach dem unter Bild 3.12 aufgeführten Schlüssel unterteilt worden sind.

Tabelle 3.3: Kornfeuchtegehalt nach 150 Tagen Lagerdauer $\left(15{ }^{\circ} \mathrm{C}\right.$ Lufttemperatur und $60 \%$ rel. Luftfeuchte)

\begin{tabular}{ccc}
\hline Versuch / Probe & Messergebnis HE 90 & Messergebnis Trockenschrank \\
\hline & Kornfeuchtegehalt [\%] & Kornfeuchtegehalt [\%] \\
3.1 & 12,7 & 11,6 \\
3.2 & 12,4 & 9,9 \\
3.3 & 11,8 & 10,7 \\
3.4 & 12,6 & \\
\hline
\end{tabular}

Nach 48 Tagen Verweildauer im Klimaschrank mit einem eingestellten Luftfeuchtegehalt von $62,0 \%$ rel. Feuchte bei $30{ }^{\circ} \mathrm{C}$ Lufttemperatur lässt sich nach Goldsaat (1988) eine Gleichgewichtsfeuchte von $12,3 \%$ erwarten. Die regelmäßig durchgeführten Kontrollmessungen durch Wiegen zeigten auf, dass nach 32 Tagen nahezu konstante Massen vorlagen. Geringfügige Differenzen begründen sich auch mit schwankendem Luftdruck und sind daher unvermeidbar. Die Kontrollprobe $(3.1 \mathrm{k}-3.4 \mathrm{k})$ hat nach der Trockenschrankmethode die in Tabelle 3.4 aufgeführten Kornfeuchtegehalte ergeben:

Tabelle 3.4: Kornfeuchtegehalt nach 48 Tagen Aufenthalt im Klimaschrank $\left(30{ }^{\circ} \mathrm{C}\right.$ Lufttemperatur und $62,0 \%$ rel. Luftfeuchte)

\begin{tabular}{cc}
\hline Probe Nr. & Kornfeuchtegehalt [\%] \\
\hline $3.1 \mathrm{k}$ & 11,74 \\
$3.2 \mathrm{k}$ & 11,37 \\
$3.3 \mathrm{k}$ & 11,13 \\
$3.4 \mathrm{k}$ & 10,80 \\
\hline
\end{tabular}

Entsprechend der Aufschlüsselung nach Bild 3.12 liegen insgesamt 16 Einzelproben vor. Aus jeder getrockneten Maischarge nach den Versuchen 3.1 bis 3.4 wurden ganzkörnige und gebrochene Maiskörner unterteilt, die wiederum jeweils in Glasgefäße mit übersättigten und untersättigen Salzlösungen eingebracht wurden. Nach ca. 35 Tagen (840 Stunden) waren keine Massenveränderungen mehr messbar. Geringfügige Veränderungen begründen sich auch hier 
in einem nicht konstanten Luftdruck und sind bei der vorstehenden Versuchsanordnung unvermeidbar. Bild 3.25 verdeutlicht in Abhängigkeit der Zeit die relativen Masseänderungen der Einzelproben. Der Bezug zur relativen Masseänderung hat den Vorteil, dass sich hierdurch die gleichen qualitativen Aussagen hinsichtlich des Sorptionsverhaltens der Einzelproben zueinander machen lassen und zudem Unterschiede durch verschiedene Startfeuchtegehalte (Tabelle 3.4) kompensiert werden. Die Einzelproben sind dadurch direkt vergleichbar. Nach mathematischer Umstellung der Formel (7) lässt sich die eingetretene Gutfeuchte (Endfeuchtegehalt $\left.\mathrm{F}_{\mathrm{E}}\right)$ mit dem Anfangsfeuchtegehalt $\left(\mathrm{F}_{\mathrm{A}}\right)$, der Anfangsmasse $\left(\mathrm{m}_{\mathrm{F}}\right)$ und der Massedifferenz ( $\mathrm{m}_{\mathrm{H} 2 \mathrm{O}}$ ) errechnen und mit Ergebnissen der Trockenschrankmethode vergleichen.

Bei Desorption: $\quad \mathrm{F}_{\mathrm{E}}=\frac{\mathrm{m}_{\mathrm{F}} \cdot \mathrm{F}_{\mathrm{A}}-100 \cdot \mathrm{m}_{\mathrm{H} 2 \mathrm{O}}}{\mathrm{m}_{\mathrm{F}}-\mathrm{m}_{\mathrm{H} 2 \mathrm{O}}}$

Bei Adsorption: $\quad \mathrm{F}_{\mathrm{E}}=\frac{\mathrm{m}_{\mathrm{F}} \cdot \mathrm{F}_{\mathrm{A}}+100 \cdot \mathrm{m}_{\mathrm{H} 2 \mathrm{O}}}{\mathrm{m}_{\mathrm{F}}+\mathrm{m}_{\mathrm{H} 2 \mathrm{O}}}$

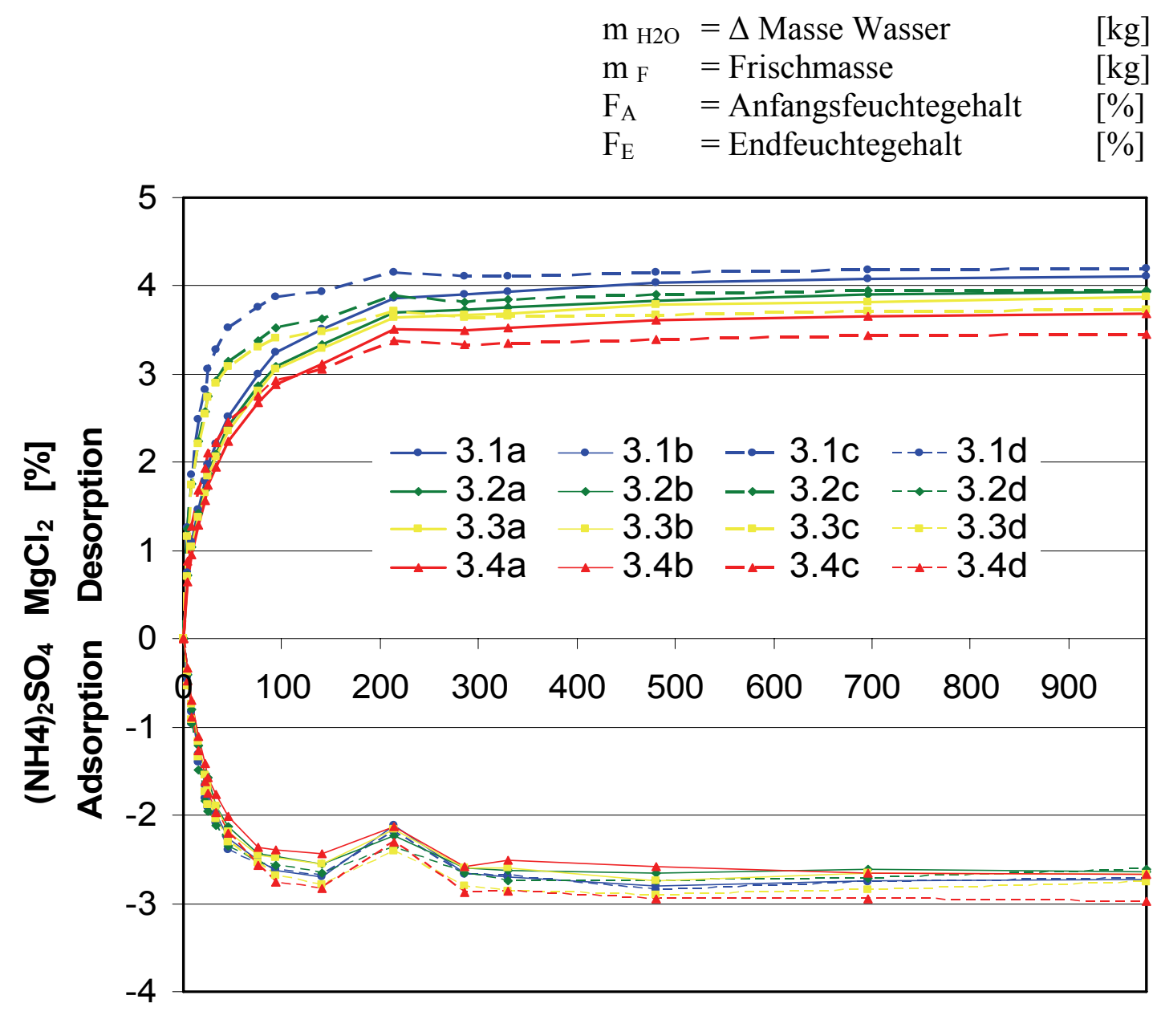

Einwirkdauer der gesättigen Salzlösung bei $30^{\circ} \mathrm{C}[\mathrm{h}]$

Bild 3.25: Relative Masseänderung der Einzelproben in Sorptionslösungen Versuche 3.1a bis 3.4d 
Die graphische Darstellung (Bild 3.25) der Masseänderungen der jeweiligen Proben in den mit gesättigten Salzlösungen aus Magnesiumchlorid bzw. Ammoniumsulfat befindlichen Einzelgläsern zeigt auf, dass sich etwa nach 300 Stunden Gleichgewichtszustände sowohl hinsichtlich der Desorption als auch der Adsorption eingestellt haben. Auffällig ist, dass in den ersten 100 Stunden nach Versuchsbeginn die größten Masseänderungen auftraten.

Die Proben über der Ammoniumsulfatlösung zeigen aufgrund der nahe beieinander liegenden Kurven ein weitgehend ähnliches Befeuchtungsverhalten in der Atmosphäre bei $30{ }^{\circ} \mathrm{C}$ und $80 \%$ relativer Luftfeuchte, wobei durchgängig Unterschiede hinsichtlich des Gleichgewichtsfeuchtegehaltes der vier Versuche bestehen. Die vorübergehende einheitliche Verringerung der Masse aller Proben in Ammoniumsulfatlösung - also der Reduzierung des Feuchtegehaltes - nach etwa 200 Stunden ist auffällig, lässt sich aber nicht schlüssig erklären. Mitunter sind Veränderungen der eingestellten Raumluftbedingungen im Innern des Klimaschrankes aufgetreten. Ein Vertauschen von Proben während des Wiegens erscheint ausgeschlossen, weil von der Gewichtsreduzierung alle acht Proben in der Ammoniumsulfatlösung gleichermaßen betroffen sind, nicht aber die acht Proben in der Magnesiumchloridlösung. Gründe, die sich durch die Struktur der Maiskörner erklären, sind unwahrscheinlich. Alle Proben sind in einem Klimaschrank untergebracht gewesen.

Die Proben über der Magnesiumchloridlösung zeigen innerhalb der ersten 200 Stunden nach Versuchsbeginn ein deutlich voneinander abweichendes Entfeuchtungsverhalten. Es zeigt sich bei einer Atmosphäre innerhalb der Probegläser mit $30{ }^{\circ} \mathrm{C}$ und $33 \%$ relativer Luftfeuchte, dass die Proben in der Reihenfolge von der längsten bis zur kürzesten Trocknungsdauer (Versuch 3.1 bis 3.4) hinsichtlich der Desorption - also im Prinzip einer weiterführenden Trocknung bis zum Gleichgewichtsfeuchtegehalt schneller reagieren, als die Proben mit höherer Trocknungsgeschwindigkeit. Die relativen Masseänderungen zeigen sich bei gleicher Versuchsdauer bei den Proben über der Magnesiumchloridlösung mit der hierdurch einhergehenden Entfeuchtung etwa ein Prozentpunkt größer als bei der Befeuchtung der Körnermaisproben über der Ammoniumsulfatlösung.

\subsection{Diskussion}

Die bisherigen Versuche zur Optimierung von Trocknungsprozessen mit mikrowellenunterstützter Warmlufttrocknung folgten bislang der These, dass ein Trocknungsprozess zunächst mit einer möglichst preiswerten, und auch preiswert am Produkt 
wirkender Energie erfolgen soll (Meisel 1976, Münsterer 2000, Tarsa 1991). Dieses ist mit konventioneller Warmlufttrocknung bei vielen Stoffen, insbesondere bei Agrargütern der Primärerzeugung gegeben. Erst bei nachlassender Trocknungsgeschwindigkeit der Konvektionstrocknung durch verringerte Wärmeleitung in das Produkt, Verringerung der Diffusionsfähigkeit der Produktoberfläche und schwindendem Feuchtegehalt sei dann eine zum Beispiel durch Mikrowellen anders wirksam werdende Energieeinkopplung vorteilhaft. Hierbei ist aber auch zu berücksichtigen, dass Stoffe mit geringem Wassergehalt für die Erwärmung im Mikrowellenfeld aufgrund der schlechter werdenden Energieumsetzung ungünstig zu bewerten sind (Hörsten von 1995). Das spricht gegen einen beginnenden Mikrowelleneinsatz zum Trocknungsende bei der Kombinationstrocknung. Mühlbauer (1979) stellt in seinen Versuchen zur Trocknung von Körnermais mit einer Kombination aus einer Gleichstrom- und Gegenstrom-Warmlufttrocknungsanlage fest, dass im Vergleich zu einem einstufigen Gleichstromtrockner eine Leistungserhöhung von 20 bis 100 Prozentpunkte möglich ist. Die Kombinationsanlage erfordere einen 10 bis 20 Prozentpunkte geringeren Trocknungsenergiebedarf. Aus diesen Untersuchungen wird deutlich, welchem verfahrenstechnischen Einfluss die Anwendung der Warmlufttrocknung unterliegt. Hierdurch leitet sich aber auch ab, dass die möglichen Vorteile einer mikrowellenunterstützten Trocknung nur dann wirksam werden können, wenn das Optimierungspotential konvektiver Trocknungstechnik ausgeschöpft ist. In seinen Ausführungen zu Mikrowellenenergie in der Trocknungstechnik führt Gehrmann (2005) aus, dass international viel Forschungsarbeit in industrielle Anwendung von Mikrowellenenergie investiert wird. Da diese Technologie ständig mit den system-immanenten Problemen der unkontrollierten Ankopplung (hot spots), Funkenentladungen (Verblitzen) und inhomogener Energieverteilung zu kämpfen hat, wird diese Technologie für jeden neuen Einsatzfall Entwicklungsbedarf benötigen. Für Arbeiten im Pharma- und Lebensmittelbereich werden diese Arbeiten am ehesten relevant sein (Gehrmann 2005).

Wenn Mikrowellentrockner nach thermodynamischen Überlegungen eingesetzt werden, eignet sich hierzu als Regelindikator der Abluftfeuchtegehalt. Sinkt der Abluftfeuchtegehalt im Verlauf der Trocknung, kann durch den Einsatz von Mikrowellenenergie ein höherer Dampfdruck im Innern des Trocknungsgutes aufgebaut werden. Hierbei wird die Mikrowellenenergie nicht durch Produktwiderstände (Reflektion, Durchlässigkeit, Absorption) in ihrer Wirksamkeit und Wirkrichtung beeinflusst. Bei den durchgeführten Versuchen 3.1 bis 3.4 zur Trocknung von Körnermais zeigt sich, wie sich die Trocknungsdauer bei zunehmendem Mikrowelleneinsatz verkürzt. 


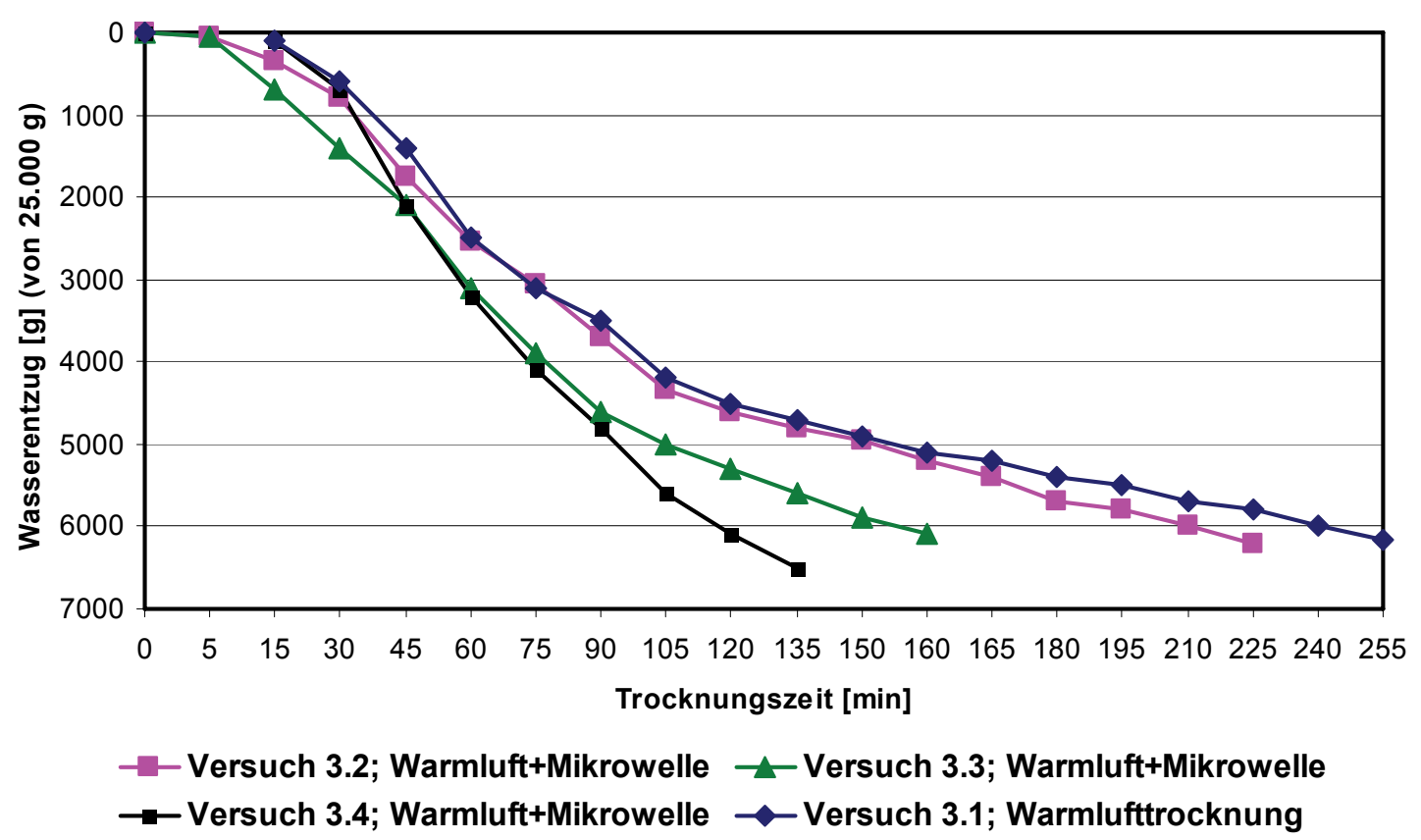

Bild 3.26: Wasserentzug je Trocknungsminute, Versuch 3.1 bis 3.4

Bei der Warmlufttrocknung wird der zum Trocknen erforderliche Dampfdruck durch Konvektion und im Produkt durch Wärmeleitung aufgebaut. Im Unterschied dazu erfolgt bei der Mikrowellentrocknung ein Aufbau des Dampfdruckes durch eine Energieumsetzung in Wärme direkt in den im Produkt enthaltenen Wassermolekülen. Eine nachlassende Wirkung der Konvektionstrocknung zeigt sich durch eine abnehmende Trocknungsgeschwindigkeit. Die Prozesse des Energieeintrages durch Konvektion und des Feuchtigkeitsaustrages im Produkt sind gegenläufig und beeinflussen sich in ihrer Ausprägung zueinander derart, das es daher angebracht ist, die Mikrowellenapplikation bereits schon dann beginnen zu lassen, bevor eine Abnahme der Trocknungsgeschwindigkeit eintritt.

Die Ergebnisse der Trocknungsversuche zeigen durch die computertomographischen Bilder, dass das Einkoppeln von Mikrowellen zum Unterstützen von Trocknungsprozessen zu träge und auch nach jetzigen Wissenstand deutlich zu spät erfolgt, wenn als Einschaltkriterium für die Mikrowellenapplikation der Abluftfeuchtegehalt verwendet wird. Eine Zunahme der im Produkt wirkenden Mikrowellenenergie führt zu hohen Dampfdrücken. Das zeigt sich an der Ausbildung einer größer werdenden Verästelungsstruktur der korninneren Hohlräume und an blasenartigen Hohlräumen unter der Kornschale, ohne dass diese reißt (Bild 3.31). Der Mikrowelleneinsatz bewirkt eine deutliche Verkürzung der Trocknungsdauer (Bild 3.26). Um eine Mikrowellenapplikation vorteilhaft zur Körnermaistrocknung einsetzen zu können, ist die Trocknungsführung bzw. -steuerung hieran anzupassen. Der für die Warmlufttrocknung als 
eine der klassischen trocknungstechnischen Regelgrößen verwendbare Abluftfeuchtegehalt ist für eine Kombinationstrocknung mit Mikrowellen als alleiniges Steuerungskriterium zu träge. Dieses zeigt sich an den Versuchen 3.2 bis 3.4 durch eine hohe Ablufttemperatur zum Trocknungsende. Eine hohe Ablufttemperatur lässt auch auf eine hohe Korntemperatur schließen. Aus theoretischen Überlegungen und Versuchen erklärt Lücke (1992), dass sich bei richtiger Prozessführung einer Kombinationstrocknung mit angepasstem Mikrowelleneinsatz zum Trocknungsende eine tiefere Korntemperatur einstellt, als dieses bei der reinen Warmlufttrocknung zu beobachten ist. Diese Überlegungen werden durch die Auswertung der computertomographisch vermessenen Maiskörner unterstützt.

Die Analyse der computertomographischen Vermessung zeigt bei dem eingesetzten, erntefeuchten Körnermais (Bild 3.27) bereits ein Hohlraumvolumen von 2,89 \%. Das durchschnittliche Kornvolumen des Einzelkorns beträgt 335,82 $\mathrm{mm}^{3}$.

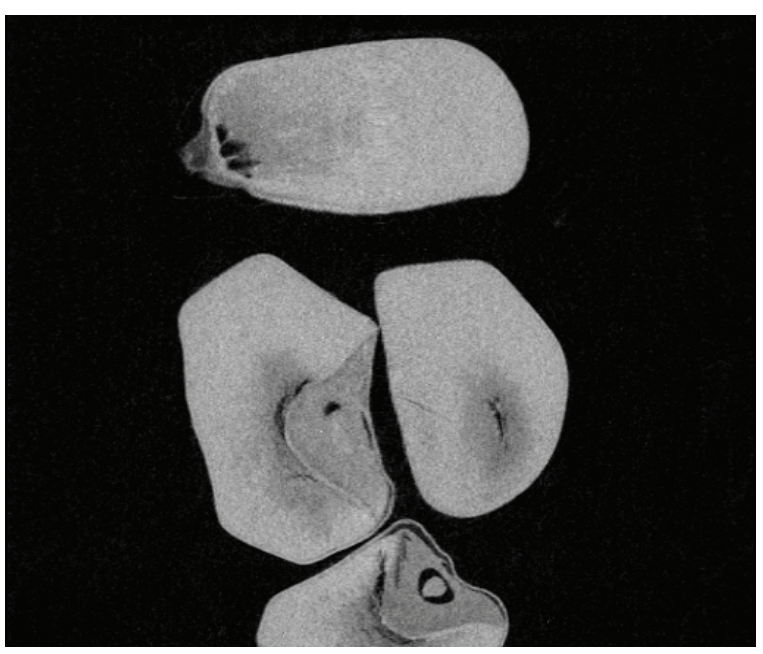

Bild 3.27: CT-Aufnahme, Körnermais feucht

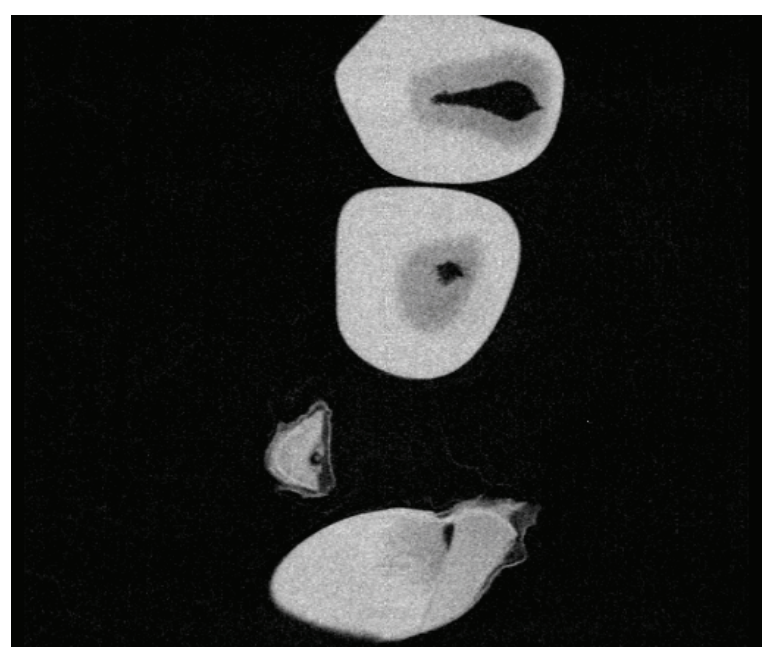

Bild 3.28: CT-Aufnahme, Körnermais luftgetrocknet $\left(20{ }^{\circ} \mathrm{C}, 65 \%\right.$ rel. Luftfeuchte)

Körnermais, der für Saatzwecke verwendet werden soll, wird typischerweise unter raumluftähnlichen Bedingungen getrocknet (Bild 3.28). Hierbei wird die Trocknungsluft auf maximal $30{ }^{\circ} \mathrm{C}$ angewärmt. Bei einem Endfeuchtegehalt von 14,8\% weist diese Probe im lagerstabilen Zustand bereits einen Hohlraumanteil von 4,45\% auf, wobei sich das durchschnittliche Kornvolumen bei der vorliegenden Partie auf 264,55 $\mathrm{mm}^{3}$ verringert hat. Dieses entspricht einer Volumenverringerung auf 78,8\%. Interessant ist, dass sich bei der Warmlufttrocknung unter den Bedingungen der guten fachlichen Praxis nach der Trocknung mit einer Warmlufttemperatur von ca. $125{ }^{\circ} \mathrm{C}$ der durchschnittliche Hohlraumanteil sich auf $8,89 \%$ nahezu verdoppelt hat, hingegen das durchschnittliche Kornvolumen $284,10 \mathrm{~mm}^{3}$ 
beträgt. Im Verhältnis zum Feuchtmais führt die konventionelle Warmlufttrocknung bei diesem Versuch zu einer Volumenverringerung auf 84,7\%.

Die Verfahrenskombination der Warmluft- und Mikrowellentrocknung führt bei der Applikation der Mikrowellenenergie nach thermodynamischen Überlegungen zu keiner Erhöhung des Hohlraumvolumens im Korn. Bei den Versuchen 3.2 und 3.3 liegen die Werte mit 7,74 \% bzw. 8,56 \% sogar geringfügig unter dem der reinen Warmlufttrocknung, obwohl die Mikrowellenapplikation eine deutliche Beschleunigung der Trocknung bewirkt (Bild 3.29). Demgegenüber ist aber zu sehen, dass das nach der Trocknung vorhandene durchschnittliche Kornvolumen mit 291,11 $\mathrm{mm}^{3}$ (Versuch 3.2: 86,8 \%) bzw. 282,28 $\mathrm{mm}^{3}$ (Versuch 3.3: 84,1\%) vergleichbar bleibt.

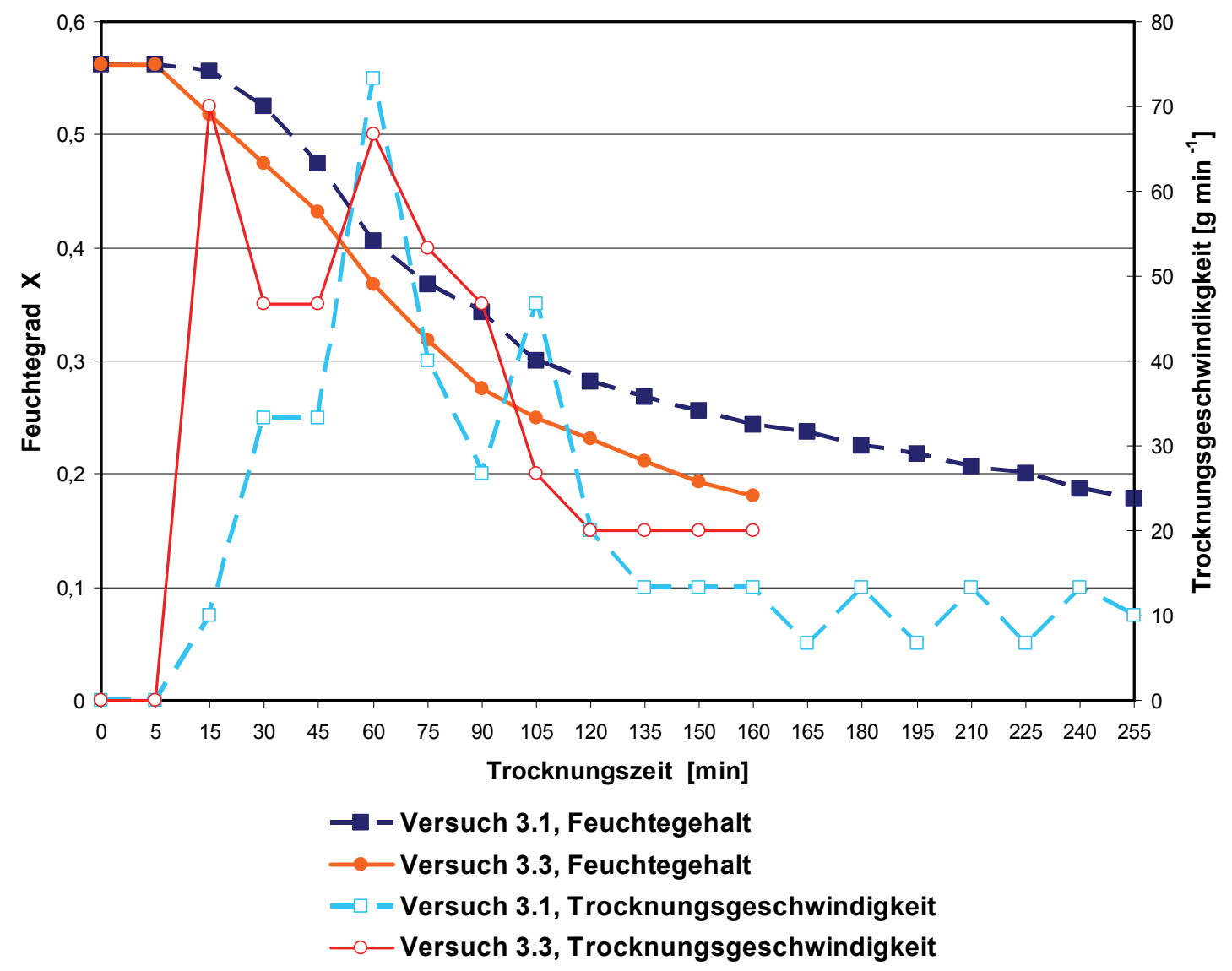

Bild 3.29: Feuchtegrad und Trocknungsgeschwindigkeit aus Versuch 3.1 und 3.3 aufgetragen über der Trocknungsdauer.

Die Trocknungsparameter des Versuchs 3.4 sind auf einen übermäßig hohen Mikrowellenenergieeinsatz ausgerichtet (Bild 3.30). Einerseits sollen größtmögliche Trocknungsgeschwindigkeit erreicht und andererseits auch möglichst signifikante physikalische Strukturänderungen im Maiskorn mit den Möglichkeiten des Chargenmischertrockners erreicht werden. 


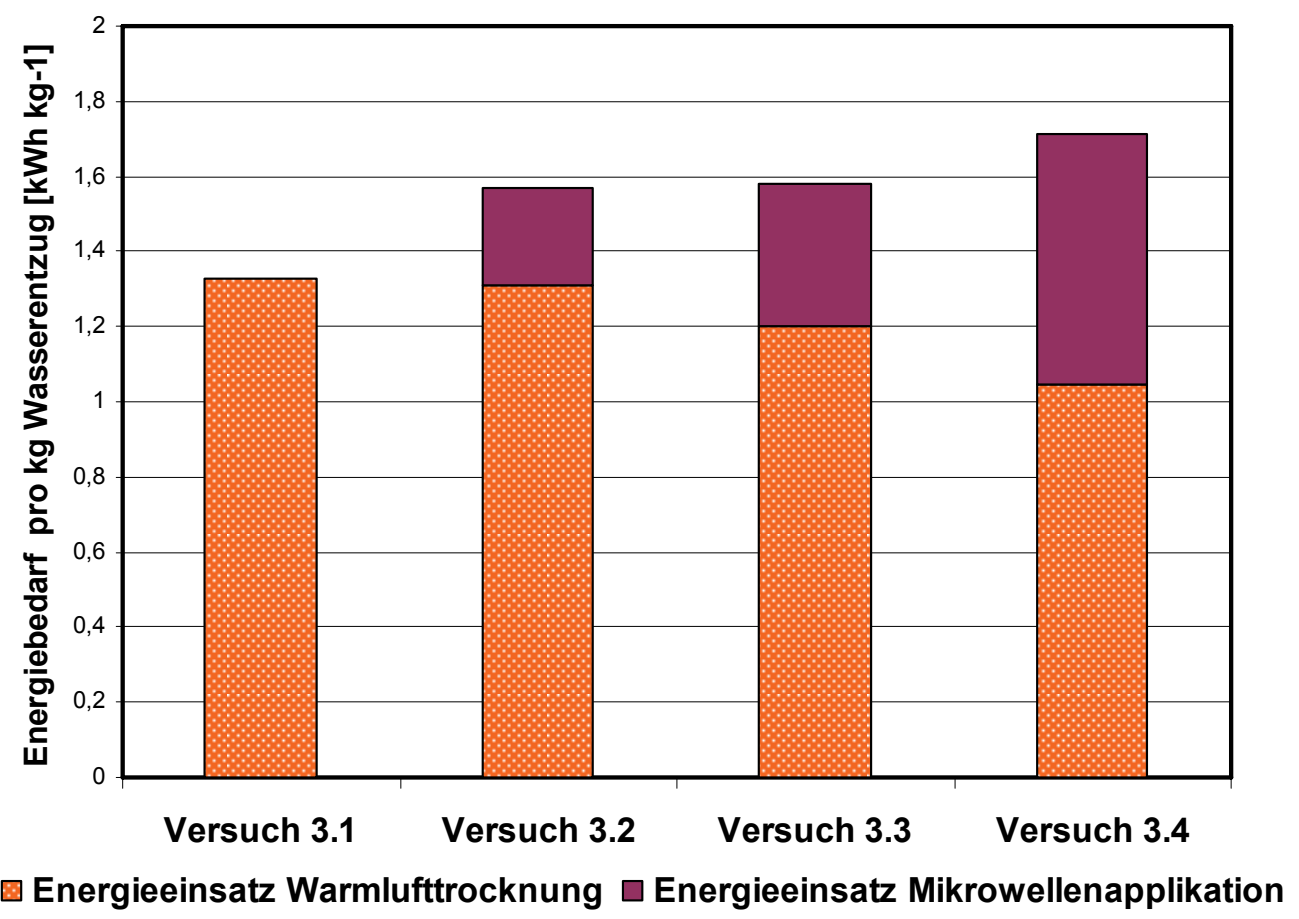

Bild 3.30: Trocknungsenergieeinsatz im Vergleich, Versuche 3.1 bis 3.4

Bei Versuch 3.4 ist im Vergleich $\mathrm{zu}$ Versuch 3.1 für die Trocknung von einem Anfangsfeuchtegehalt von 37,5 \% auf einen Endfeuchtegehalt von $14,1 \%$ eine Trocknungsdauer von 140 Minuten erforderlich. Das ergibt eine Reduzierung der Trocknungsdauer um 48,2\%, wobei sich aber der spezifische Trocknungsenergieaufwand um $27 \%$ auf 1,69 $\mathrm{kWh} \mathrm{kg}^{-1}$ Wasserentzug erhöht hat. Der durchschnittliche Hohlraumanteil in den Maiskörnern beträgt bei dem Versuch 3.4 im getrockneten Zustand 10,3\% bei einem Kornvolumen von 279,97 $\mathrm{mm}^{3}$. Bei der Detailbetrachtung von CT-Aufnahmen lässt sich bei einzelnen Körnern aber im Gegensatz zu dem Versuch 3.1 (Bild 3.32), Versuch 3.2 und Versuch 3.3 eine erhöhte Anzahl kleiner Hohlräume erkennen. Hieraus lässt sich schließen, dass temporär ein zu hoher Dampfdruck im Korninnern vorhanden gewesen sein muss, sodass die Aufweitung der vorhandenen Verästelungen nicht mehr ausreichend schnell hat erfolgen können (Bild 3.31). Bei einigen Körnern führte dieses $\mathrm{zu}$ Rissen in der Samenschale. Initialisierend für Risse der Samenschale können aber auch mechanische Einwirkungen während des Mähdruschs sein, die sich erst während der Trocknung durch den Aufbau von Dampfdruck offenbaren. Hierdurch verursachte Störungen können auch bei der Warmlufttrocknung auftreten (Mühlbauer 1974). Mitunter können auch während des Warmluft-Trocknungsprozesses durch Wirken des Dampfdrucks im Korn Spannungen auftreten, die zum Platzen der Körner und Bilden von Rissen führen (Maltry et al. 1975). Im Verhältnis zu der auch bei reiner Warmlufttrocknung unter praxisrelevanten 
Betriebsparametern auftretenden Hohlraumausbildung von etwa 8,8 \% erscheint eine Erhöhung auf 10,3 \% entsprechend der Durchführung von Versuch 3.4 nicht unverhältnismäßig hoch.

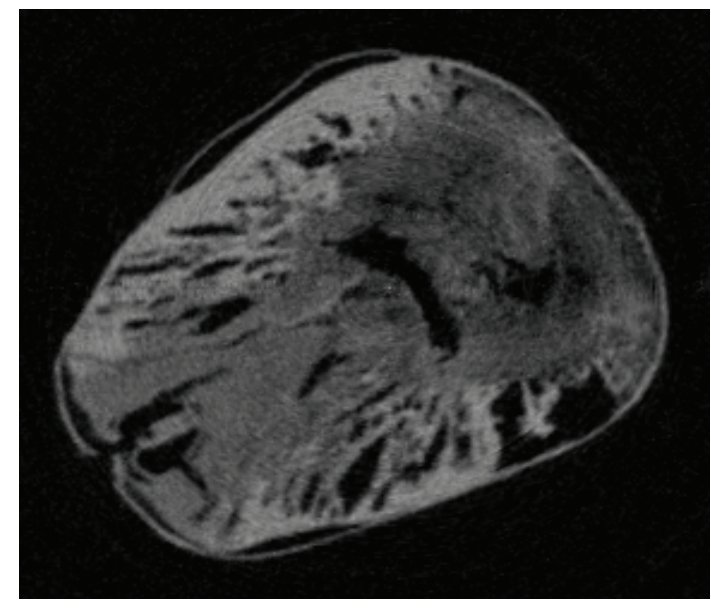

Bild 3.31: CT-Schnitt, ausgewähltes Korn aus Versuch 3.4

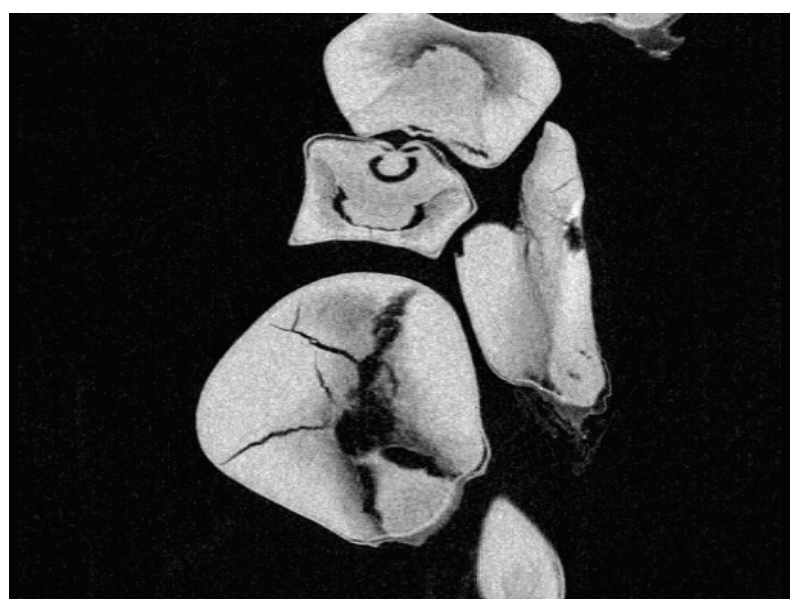

Bild 3.32: CT-Schnitt, ausgewähltes Korn aus Versuch 3.1 (Warmlufttrocknung)

Häufig lässt sich auch eine Ablösung der Samenschale vom Endosperm beobachten, ohne dass die Samenschale aufreist. Es ist zunächst anzunehmen, dass bei dieser Form der mikrowellenunterstützten Trocknung die äußeren Bereiche des Maiskorns feuchter und damit elastischer bleiben und somit ein Aufreißen der Samenschale unterbleibt. Des Weiteren tritt mit fortschreitender Trocknung eine Schrumpfung der korninneren Bestandteile ein, so dass sich hierdurch in gewisser Weise eine innere Hohlraumvergrößerung ermöglicht, ohne dass sich die Samenschale ihrerseits deutlich aufweiten muss. Erst durch eine fortwährende Betrachtung aller CT-Schnitte eines Maiskorns wird deutlich, dass die ausgebildeten Hohlräume nicht wie einzelne, abgeschlossene Hohlkammern zu sehen sind, sondern eine ineinander greifende Struktur aufweisen, wodurch während der Trocknung ein Ausgleich des Dampfdruckes im Innern des Korns unterstützt wird. Es zeigt sich, dass unterschiedliche Trocknungsintensitäten zu unterschiedlichen räumlichen Änderungen der Struktur im Innern von Maiskörnern führen. Diese Prozesse mit Hilfe von Computertomographie zu visualisieren ist neu. Um der Frage nachzugehen, ob die durch die Mikro-Computertomographie aufgezeigten Änderungen der Struktur aufgrund unterschiedlicher Trocknungsintensitäten auch nach der Trocknung Einfluss auf den Körnermais behalten, sind Sorptionsversuche durchgeführt worden. Es wird deutlich, dass sich mit zunehmendem Einsatz von Mikrowellenenergie ergänzend zur Konvektionstrocknung das Sorptionsverhalten dahingehend ändert, dass sich sowohl die Feuchtigkeitsaufnahme als auch -abgabe gehemmter erfolgt. Dieses begründet sich offenbar mit einer veränderten Kapillar- und Hohlraumstruktur, obwohl sich der das FestkörperHohlraum Verhältnis als solches nicht signifikant ändert. Im Ergebnis führt ein zunehmender 
Einsatz von Mikrowellenenergie nicht primär zu größeren Hohlräumen, aber zur Ausprägung von deutlich mehr Hohlräumen, auch zu kleineren. Erst eine übermäßige Trocknungsintensität führt zu einer deutlichen Vergrößerung und Anzahl von Hohlräumen. Dieser Prozess führt dann zu Pop-Corn ähnlichen Ausbildungen (Bützer 2005). In beiden Fällen verschlechtern sich - zwar aus unterschiedlichen Gründen - die kapillaren Eigenschaften.

Nach den Ausführungen von Mühlbauer (1974) ließ sich in seinen Versuchen nicht feststellen, dass entgegen früherer Annahmen die Kornschale der Feuchtebewegung einen erheblichen Widerstand entgegensetzt, weil Körner mit und ohne Schale das gleiche Trocknungsverhalten aufweisen. Diese Feststellung steht insofern nicht im Widerspruch zu den Erkenntnissen des Sorptionsverhaltens der Proben 3.1a bis 3.4d, als dass gebrochener und ganzkörniger Mais keine signifikanten Unterschiede hinsichtlich des Sorptionsverhaltens zeigen. Im Vergleich zur Trocknung erfolgt die durch unterschiedliche Salzlösungen mögliche Feuchtediffusion aber sehr langsam, so dass die Kornschale nicht als Diffusionsbarriere wirkt. Im Gegensatz dazu wird aus Bild 3.31 deutlich, dass bei einem hohen Energieeintrag während der Trocknung die Schale schon als Diffusionsbarriere wirkt. Das zeigt sich an blasenartigen Hohlräumen unter der Kornschale. Daraus leitet sich ab, dass mit der Warmlufttrocknung allein die Grenzen einer produktverträglichen Trocknungsgeschwindigkeit nicht erreicht werden, es mit einer unterstützenden Mikrowellenapplikation aber möglich ist, die Trocknungsgeschwindigkeiten soweit zu erhöhen, dass die Unversehrtheit des Korns nicht mehr gegeben ist.

In den Untersuchungen zur mikrowellenunterstützten Vakuumkonvektionstrocknung an Rindfleischklößchen kommt Heindl (1993) zu dem Ergebnis, dass eine schonende und gleichmäßige Trocknung durch niedrige Wasserverdampfungstemperaturen von $46{ }^{\circ} \mathrm{C}$ bzw. 76 ${ }^{\circ} \mathrm{C}$ im Vakuumbereich (100 - 400 mbar) erreicht wird. Die Produkttemperaturen zum Trocknungsende bleiben dadurch niedrig. Das dem Chargenmischertrockner nachgeordnete Radialgebläse führt durch die saugende Betriebsweise $\mathrm{zu}$ einem Unterdruck in der Trockengutkammer von ca. ein bis zwei Millibar. Hierdurch lassen sich aber noch keine nennenswerten Eigenschaften im Sinne einer Vakuumtrocknung erreichen. Wesentlicher verfahrenstechnischer Vorteil des im Saugverfahren betriebenen Chargenmischertrockners ist die Reinhaltung der Anlage und der Anlagenumgebung im Betrieb sowie eine gute messtechnische Erfassung der Prozessluftzustände. Es ist anzunehmen, dass eine deutliche Erhöhung des Betriebsvakuums bei einer mikrowellenunterstützten Konvektionstrocknung auch bei der Körnermaistrocknung $\mathrm{zu}$ einem tieferen Guttemperaturniveau führt. Diese Zusammenhänge können nur in weiteren Versuchen erforscht werden. 


\subsection{Schlussfolgerungen / Ausblick}

Die Versuche zielen darauf ab, die technische Machbarkeit einer Verfahrenskombination zur Trocknung von Körnermais mit Warmluft und Mikrowellen zu untersuchen und den Einfluss physikalisch unterschiedlich wirkender Energie auf das Trocknungsgut zu erarbeiten. Hierzu werden Strukturzustände in Maiskörnern mit Hilfe der Computertomographie visualisiert. Erkenntnisse der Computertomographie korrespondieren mit den Ergebnissen aus den Sorptionsversuchen. Aus den Trocknungsversuchen leitet sich ab, dass weitere verfahrenstechnische Optimierungen erforderlich sind, damit eine mikrowellenunterstützte Warmlufttrocknung zur Körnermaistrocknung vernünftig eingesetzt werden kann. Eine Beschleunigung der Trocknung durch den unterstützenden Einsatz von Mikrowellenenergie führt zu einem insgesamt höheren spezifischen Trocknungsenergiebedarf, wenn die Mikrowellenapplikation nicht hinreichend angepasst erfolgt ist. Durch die computertomographischen Aufnahmen zeigt sich, dass Strukturänderungen in Maiskörnern bei jeder Trocknung auftreten, auch bei der Raumlufttrocknung, die der natürlichen Trocknung sehr nahe kommt. Die Warmlufttrocknung mit einer praxisbewährten Zulufttemperatur von 125 ${ }^{\circ} \mathrm{C}$ führt bereits $\mathrm{zu}$ einer Hohlraumbildung (8,89\%) im Korninnern, die zum Ende der Trocknung etwa den zweifachen Wert der Raumlufttrocknung (4,45\%) aufweist. Durch die Versuche 3.2 und 3.3 wird deutlich, dass eine Mikrowellenapplikation mit der hier gewählten Sendeleistung zu keinen auffällig anderen Volumenverhältnissen (Festkörper - Hohlraum) führt als bei der Warmlufttrocknung. Hieraus leitet sich ab, dass zur Unterstützung der Warmlufttrocknung eine Mikrowellenapplikation mit angepasster Sendeleistung vorteilhaft zur Maistrocknung eingesetzt werden kann.

Sensorische Prüfungen, stoffinhaltliche Untersuchungen, Keimproben, Fütterungsversuche oder mikroskopische Analysen sind zur Qualitätsbewertung landwirtschaftlicher Früchte bewährt. Dennoch lässt sich nur durch eine zerstörungsfreie Untersuchung in Verbindung mit dem Trocknungsprozess aufzeigen, wie ein auf die Körnermaistrocknung ausgerichtetes Verfahren zu optimieren ist. Eine zerstörungsfreie Visualisierung, wie das Trocknungsgut auf das Einwirken von Trocknungsenergie reagiert, ermöglicht eine anders begründete Bewertung von Trocknungsprozessen. Dieses wird für weitere Forschungen und Entwicklungen in der landwirtschaftlichen Trocknungstechnik bedeutsam sein. 


\section{Epilog}

Verschiedene wissenschaftliche Untersuchungen befassen sich in unterschiedlichen Fragestellungen mit Mikrowellentechnologie. Ebenso sind Mikrowellenanlagen sowohl in Hauhalthalten und industriell genutzten Anlagen zur Trocknung, Wärmebehandlung von Früchten, Hölzern oder Industrierohstoffen oder auch für einige messtechnische Anwendungen bewährt. Im Vergleich zur reinen Konvektionstrocknung sind bei der Mikrowellenanwendung eine aufwendigere mechanisch-technische Apparatur, zusätzliche steuer- und regelungstechnische Elemente sowie sicherheitstechnische Einrichtungen erforderlich. Deshalb hat sich diese Technik bislang dort etabliert, wo Behandlungsabläufe wie Trocknen ganzjährig erforderlich sind, oder die in Verbindung mit dem Verfahren einhergehende Wertschöpfung durch besser einstellbare Produkteigenschaften, Reduzierung des Trocknungsenergiebedarfs oder Verfahrensbeschleunigung gegeben ist. Trocknungsanlagen zur Körnermaistrocknung sind nur in der Erntekampagne, dass heißt nur ein bis zwei Monate jährlich im Einsatz. Deshalb dominieren in der Praxis bislang Warmlufttrocknungsanlagen, die auch hinsichtlich ihrer technischen Ausstattung an den temporären Einsatz angepasst sind. Obwohl die theoretischen Zusammenhänge konventioneller Warmlufttrocknungssysteme für die Körnermaistrocknung weitgehend erforscht und diese Ergebnisse auch in der Praxis umsetzbar sind, zeigt sich aber dort oft noch ein hohes Potential an möglicher Energieeinsparung und Verfahrensoptimierung. Aufgrund tendenziell wachsender Erträge, witterungs- oder sortenzuchtbedingt enger werdender Erntezeiträume und vermarktungstechnischen Strategien zeichnen sich schon jetzt in der Praxis teilweise deutliche Trocknungsengpässe zur Erntezeit ab. Dieses ist umso kritischer zu sehen, als das nach heutigem Wissen die Produktqualität entscheidend durch eine zügige und richtige Nacherntebehandlung beeinflusst wird und dieses ein zunehmend wichtiger werdendes Vermarktungskriterium wird.

Durch eine mikrowellenunterstützte Warmlufttrocknung lassen sich im Vergleich zur reinen Warmlufttrocknung deutlich kürzere Trocknungszeiten bei einem geringeren spezifischen Trocknungsenergiebedarf erreichen. Die durchgeführten Versuche zeigen aber auch, dass eine Verringerung des spezifischen Trocknungsenergiebedarfes nur dann erreicht werden kann, wenn die Mikrowellenapplikation optimal auf das Trocknungsgut und den Trocknungsprozess ausgerichtet ist. Für die praktische Anwendung heißt das, dass es für eine optimale Anlagenkonfiguration erforderlich ist, unterschiedliche Vorgänge während der Konservierung mit dem Oberbegriff Trocknen $\mathrm{zu}$ trennen. Vorteile einer unterstützenden Mikrowellenanwendung lassen sich nur dann umsetzen, wenn die Optimierungspotentiale der 
Warmlufttrocknung erschöpft sind. Für den vorteilhaften Einsatz von Mikrowellen ist deren räumliche und verfahrenstechnische Einkopplung in den Trocknungsprozess wichtig.

Um eine gleichmäßige Produkterwärmung durch die Mikrowellenenergie zu erreichen, ist eine ausreichend hohe Produktbewegung während der Trocknung erforderlich. Dieses bedingt sich durch die Ausbreitungsgeometrie der Mikrowellen im Trockenraum, welche durch die Raumkonstruktion und die konkreten Trockenguteigenschaften bestimmt werden. Hierzu notwendige Bewegungsgeschwindigkeiten sind bei den heute bewährten Trocknungssystemen zur Maistrocknung unüblich, allein schon deshalb, um keinen unnötig hohen Produktabrieb zu provozieren und keine unnötige mechanische Antriebsleistung $\mathrm{zu}$ installieren. Die bei Warmlufttrocknungsanlagen vorgesehenen Bewegungsgeschwindigkeiten orientieren sich an dem Niveau, welches zum gleichmäßigen Wärmeeintrag und zur Feuchtigkeitsabfuhr erforderlich ist. Daraus lässt sich für weitere Entwicklungen die Empfehlung aussprechen, dass es für eine mikrowellenunterstützte Warmlufttrocknung anbietet, das Trocknungssystem aus unterschiedlichen Teilelementen bestehen zu lassen, um den Trocknungsvorgang optimal an die Erfordernisse des Trocknungsgutes und die jeweilige Form und Zustand der Trocknungsenergie anpassen zu können. Derzeitige Warmlufttrocknungssysteme sehen das nur bedingt vor. Im Ergebnis führt eine pauschalierende Trocknung eines auf alle Körner konstant wirkenden Energieangebotes in einem Trockner dazu, dass unabhängig von Feuchtegehalt und Feuchteverteilung in der Trockengutschüttung und ebenso im Einzelkorn nur dadurch eine gleichmäßige Trocknung bei geringem spezifischem Energiebedarf möglich ist, indem sich hierzu während der Trocknung ein ausreichend hoher Ausgleich des Produktfeuchtegehaltes mit der hierzu erforderlichen Ausgleichsdauer - in der Trockengutschüttung überlagert. Daher lässt sich auch mit der Warmlufttrocknung unter Wahrung der Produktqualität keine weitere Verkürzung der Trocknungsdauer bei Körnermais erwarten. Eine am Korn mit hoher Temperatur wirkende Trocknungsenergie führt zu einem Aufbau von Diffusionsbarrieren an der Gutoberfläche, wodurch sich die Trocknungszeit verlängern kann. Diese Grenzen sind in der Praxis erreicht.

Bei der Mikrowellentrocknung erfolgt im Gegensatz zur Warmlufttrocknung eine Verdampfung der Feuchtigkeit im Korninnern. Die äußeren Schichten bzw. Schalen des Maiskorns bleiben bei einer Mikrowellenapplikation bis zum Ende des Trocknungsprozesses diffusionsfähiger, weil der Feuchtetransport vom Korninnern an die Oberfläche nachgeführt wird. Die Folge ist eine kürzere Trocknungszeit. Für die Frage, ob und welche physikalischen Wirkungen durch unterschiedliche Trocknungsenergieeinträge und damit verbundene 
unterschiedliche Trocknungsgeschwindigkeiten hervorgerufen werden, ist die Untersuchung von Körnermais mit einer Computertomographieanlage optimal. Durch die Erstellung dreidimensionaler computertomographischer Aufnahmen zeigt sich, dass Strukturänderungen in Maiskörnern bei jeder Trocknung auftreten, auch bei der Raumlufttrocknung, die der natürlichen Trocknung sehr nahe kommt. Die Warmlufttrocknung führt bereits zu einer Hohlraumbildung im Korninnern, die zum Ende der Trocknung etwa den zweifachen Wert der Raumlufttrocknung aufweist. Obwohl die mikrowellenunterstützte Warmlufttrocknung im Vergleich zur Warmlufttrocknung regelmäßig zu einer Verkürzung der Trocknungsdauer führt, ändert sich das prozentuale Hohlraumvolumen außer bei einer von Anfang an hoch eingestellten Mikrowellensendeleistung nicht erkennbar, wohl aber die Verästelungstruktur der Hohlräume im Korninnern. Die nach der Trocknung durchgeführten Sorptionsversuche zeigen auf, dass mit zunehmendem Einsatz von Mikrowellenenergie ergänzend zur Konvektionstrocknung sowohl die Feuchtigkeitsaufnahme als auch -abgabe gehemmter erfolgt.

Um die Wirkung unterschiedlicher Trocknungstechnologien und Energieeinträge auf Körnermais $\mathrm{zu}$ bewerten, ist zur Produktanalyse unter Qualitätsgesichtspunkten eine Kombination aus Computertomographie und Messung des Sorptionsverhaltens gut geeignet. Durch Infrarotaufnahmen lassen sich die Energieumsetzung in Wärme und deren räumliche Ausbildung im Trocknungsgut feststellen. Diese Verfahren sind deshalb für eine Bewertung so bedeutungsvoll, weil die Bemusterung zerstörungsfrei erfolgt und dennoch Rückschlüsse auf physikalische Vorgänge bzw. Zustände im Produktinnern möglich sind, die in der Form bislang nicht erkennbar waren. Folglich lassen sich mit diesen Kenntnissen Trocknungsprozesse gezielter erforschen. Bei der Weiterentwicklung von Trocknungssystemen wird der Einsatz zunehmend knapper und teurer werdender Energie neu zu bewerten sein. Hierbei steht mehr denn je die Qualität des Korn im Vordergrund. 


\section{Literaturverzeichnis}

Askeland D.R. (1996): Materialwissenschaften, Grundlagen, Übungen, Lösungen. Spektrum Akademischer Verlag, Heidelberg, 607 pp.

Beke J. (1997): Terményszárítás. Verlag Agroinform Ungarn, Budapest 1997, 228-232.

Böckelmann M.(2003): Qualitätssicherung durch dokumentierte Trocknung.

Mühle + Mischfutter, Heft 23, 689-691

Böckelmann M., Weimar R.-J., Lücke W., Hörsten von D. (2007): Der Einsatz von Mikrowellen zur Maistrocknung - Grundlagen und Laborversuche. Agrartechnische Forschung 13, 27-36.

Bützer P. (2005): Popcorn. Pädagogische Hochschule St. Gallen, Schweiz, 2-32

Ciesler B. (1986): Untersuchungen zum Trocknungsverlauf und zur Entwicklung eines Trocknungsmodells der Getreidesatztrocknung. Dissertation, Georg-August Universität Göttingen, 61-64, 83-88.

Decareau R.V. (1986): Microwave Processing and Engineering. Ellis Horwood Ltd., Chichester, 150-151.

Gassner G., Hohmann B., Deutschmann F. (1989): Mikroskopische Untersuchung pflanzlicher Lebensmittel. Gustav Fischer Verlag Stuttgart, 6-22, 41.

Gehrmann D. (2003): Trocknungstechnik. Chemie Ingenieur Technik, Ausgabe 10, 1507-1514.

Gerlach D. (1985): Das Lichtmikroskop. Georg Thieme Verlag Stuttgart, 260.

Gerecke K.H. (1990): Vademekum - Technische Werte der Getreideverarbeitung und Futtermitteltechnik. Band 1, Verlag Moritz Schäfer, 71-81.

Goldsaat (1988): Werksnormen Körnerfruchtkühlung. Bindung von Wasser an landwirtschaftliche Schüttgüter, 5. 
Gröber H., Erk S., Grigull U. (1963): Die Grundgesetze der Wärmeübertragung. 3. Auflage, Springer - Verlag, Berlin, Göttingen, Heidelberg, 139-146.

Gunasekaran, S. (1990): Grain drying using continuous and pulsed microwave energy. Drying Technology, Ausgabe 8, Verlag Marcel Dekker, USA, 1039-1047

Fanslow G.E., Saul R.A. (1971): Drying Field Corn with Microwave Power and Unheated Air. Journal of Microwave Power, Volume 6, No.3, 229-235.

Friedmann G., Bücheler E., Thurn P. (1981): Ganzkörpercomputertomographie. Gerorg Thieme Verlag Stuttgart, 2-38.

Heindl A. (1993): Mikrowellenunterstützte Vakuumkonvektionstrocknung. Dissertation, Technische Universität München, 123-130.

Heindl, A., Müller, J. (2002): Mikrowellenunterstützte Trocknung von Arznei- und Gewürzpflanzen. Arznei- \& Gewürzpflanzen - Journal of Medicinal and Spice Plants, Sonderausgabe 2002, 208-225

Hippel von A.R. (1954): Dielektric Material and Applications. MIT Press, New York, 438 pp.

Hörsten von D. (1995): Einsatz von Mikrowellenenergie und anderen thermischen Verfahren zur Abtötung von Fusarium culmorum im Weizengut, 26-36.

Kersten J., Rohde H.R., Nef E. (2004): Mischfutterherstellung - Rohware, Prozesse, Technolgie. Agri-Media Verlag Bergen, 2. Auflage, 174-177.

Kialbekov R., Hörsten von D., Lücke W., Hahn J., Frerichs L. (2006): Trocknungsverhalten und Qualitätsentwicklung von Halmgut bei Konvektionstrocknung mit Mikrowellenund Hochfrequenzvorbehandlung. Agrartechnische Forschung, Heft 1, 8-13.

Krischer O., Kast W. (1972): Trocknungstechnik, die wissenschaftliche Grundlagen der Trocknungstechnik. Springer-Verlag, Berlin, Heidelberg, New York, Band 1, 287 pp. 
Kröll K., Kast W. (1989): Trocknungstechnik. Springer Verlag, Berlin, Heidelberg, New York, 1989, Band III, 65-93.

Lücke W. (1992): Mikrowellenbehandlung pflanzlicher Produkte. Habilitation, Georg-August Universität Göttingen.

Lücke W. (2006): Microwave Drying of Agricultural Materials - Basics, Effects and Mathematical Simulation. Agricultural Engineering for a Better World, CIGR World Congress, Germany, 617 pp.

Maltry W., Pötke E., Schneider B. (1975): Landwirtschaftliche Trocknungstechnik. VEB Verlag Technik Berlin, 427-440.

Martinez R. (2001): Modelling and Simulation of the Two-Stage Rice Drying System in the Philippines. Dissertation, Stuttgart-Hohenheim, 154-156

Matthis H.J. (1956): Der Strömungswiderstand beim Belüften landwirtschaftlicher Erntegüter. VDI Forschungsbericht 454.

Mellmann J., Richter I., Maltry W. (2005): Optimierte Steuerung von Getreideschachttrocknern. Bornimer Agrartechnische Berichte, Heft 52, 5 pp.

Meisel N. (1976): Mikrowellen beim Trocknen von Teigwaren. Getreide Mehl und Brot 30, H.7, 52-62.

Metaxas A.C., Meredith R.J. (1983): Industrial Microwave Heating. Peter Peregrinus Ltd, London, UK, 27 pp.

Mohsenin N.N., Moser, E., Sinn, H. (1981): Stoffeigenschaften landwirtschaftlicher Erzeugnisse in elektromagnetischen Feldern und ihre Nutzung in der landwirtschaftlichen Verfahrenstechnik. Grundlagen Landtechnik, Band 31, 90-94

Morcos B (1986): Mikrobielle Entwicklung und Verderb bei der Konservierung und Lagerung von Getreide - Schädigungsfreie Verweilzeiten und tolerierbare Grenzen -. Dissertation, Georg-August Universität Göttingen, 10-19 
Mühlbauer W. (1974): Untersuchung über die Trocknung von Körnermais unter besonderer Berücksichtigung des Gleichstrom-Trocknungsverfahrens. Dissertation, StuttgartHohenheim.

Mühlbauer W. (1979): Entwicklung eines Gleichstrom/Gegenstrom-Durchlauftrockners für Getreide und Mais. Grundlagen der Landtechnik Band 29, Nr. 4, 131-140.

Mühlbauer, W. (1980): Stand und Entwicklungstendenzen bei der Getreidetrocknung in der Bundesrepublik Deutschland. Getreide Mehl und Brot (GM\&B), Heft 4, 131-140

Münsterer J. (2000): Erste Untersuchungen zur mikrowellenunterstützten Warmlufttrocknung von Hopfen. Hopfen-Rundschau, Nummer 8, 189-191.

Münzing K. (1995): Studien zur Weizentrocknung unter besonderer Berücksichtigung wichtiger Einflussvaraiblen für die Praxis. Dissertation, Technische Universität Berlin, 3-8.

Nemitz G. (1963): Die hygroskopischen Eigenschaften getrockneter Lebensmittel. Lebensmitteluntersuchung und Forschung. Band 123, H.1, 1-5.

Nultsch W., Grahle A. (1983: Mikroskopisch-Botanisches Praktikum. Georg Thieme Verlag Stuttgart, 74-76.

Radajewski W., Jolly P., Abawi, G.Y. (1988): Grain Drying in a Continuous Flow Drier Supplemented with a Microwave Heating System. Journal of Agricultural Engineering Research, Volume 41, 211-225.

Rademacher, T. (2003): Mähdrescher - aktuelle Modelle, Daten und Fakten. RKL-Schrift 4.1.4.1.4, Rendsburg, 620 pp

Robenek H. (1995): Mikroskopie in Forschung und Praxis. GIT Verlag GmbH Darmstadt, 339 -369 .

Raju, G.G. (2003): Dielectrics in Electric Fields. Verlag Marcel Dekker, New York-Basel, 193194 
Römpp (1995): Lexikon Chemie. 10 Auflage, Georg Thieme Verlag Stuttgart.

Püschner H. (1964): Wärme durch Mikrowellen; Grundlagen, Bauelemente, Schaltungstechnik. Philips technische Bibliothek, Eindhoven/NL, 80 pp.

Sembery, P. Vögyi, F. (1989): Moisture measurement of maize at microwave frequency. Bulletin of the University of Agriculture Science, No. 1, 157-164

Schlünder E. U. (1998): Trocknungstechnik, Script Hochschulkurs. Technische Universität Karlsruhe.

Shivhare U.S., Raghavan G.S.V., Bosisio R.G. (1994): Modelling the Drying Kinetics of Maize in a Microwave Environment. Journal of Agricultural Engineering Research, Volume 57, 199-205.

Skriegan E. (2005): Technologie der Lagerung und Nacherntebehandlung von Körnerfrüchten. Verlag Moritz Schäfer, Detmold, 138-142.

Tarsa A. (1991): Versuche zur Mikrowellentrocknung von Möhren und der Rekonstitution der Trockenprodukte. Diplomarbeit, Georg-August Universität Göttingen, 53 pp.

Toftdahl O. (1987): Grain Drying. Innovation Development Engineering ApS, Denmark, 19 pp.

Trabelsi, S., Nelson, S.O. (2003): Free-space measurement of dielectric properties of cereal grain and oilseed at microwave frequencies. Institute of Physics Publishing, Measurement Science and Technology, Ausgabe 14, 589-600

Verein Deutscher Elektrotechniker (2003): VDE Schrift 0875, Teil 11, Ausgabe August 2003

Weber D. (1995): Technische Feuchtemessung in Gasen und Festkörpern. Vulkan-Verlag Essen, 120-125.

Wieneke F. (1972): Verfahrenstechnik der Halmfutterproduktion, Universität Göttingen. 
Wieneke F. (1988): Möglichkeiten des Mikrowelleneinsatzes in der Landwirtschaft. Landtechnik 4/88, 191-192.

Zscheischler J., Estler M.C., Staudacher W., Groß F., Burgstaller G., Streyl H., Rechmann Th., (1990): Handbuch Mais - Umweltgerechter Anbau - Wirtschaftliche Verwertung. DLG Verlag Frankfurt am Main, 208-215. 


\section{Danksagung}

Die vorliegende Arbeit entstand in der Abteilung für Agrartechnik des Departments für Nutzpflanzenwissenschaften der Georg-August-Universität Göttingen in Zusammenarbeit mit dem Fachbereich Agrarwirtschaft und dem Fachbereich Elektrische Energietechnik der Fachhochschule Südwestfalen, Abteilung Soest und dem Labor für Mikrowellentechnik am Institut für Technologie- und Wissenstransfer (TWS) im Kreis Soest e.V.

Mein besonderer Dank gilt Herrn Prof. Dr. W. Lücke, der mir durch seine jederzeit gewährende Unterstützung und ständige Diskussionsbereitschaft wertvolle Anregungen gab. Ich danke ihm für die wissenschaftliche Betreuung und das mir entgegengebrachte Vertrauen. Sein Engagement hat mir die Durchführung der Arbeit in dieser Form ermöglicht.

Frau Prof. Dr. E. Pawelzik, Abteilung Qualität Pflanzlicher Erzeugnisse, gilt mein Dank für die gewährte Unterstützung und ihr stetes Interesse am Fortgang der Untersuchungen und für die Übernahme des Koreferates.

Gleichsam möchte ich Herrn PD. Dr. M. Worbes, Abteilung Pflanzenbau der Tropen und Subtropen, für sein Interesse an der Arbeit und seine Hilfsbereitschaft danken.

Herrn Dr. D. von Hörsten möchte ich stellvertretend für alle Mitarbeiterinnen und Mitarbeiter der Abteilung für Agrartechnik meinen Dank für die geleistete Unterstützung aussprechen. Als externer Doktorand hat mir die offene, konstruktive Zusammenarbeit sehr geholfen.

In besonderer Weise hat Herr Prof. Dr. R.- J. Weimar, Fachhochschule Südwestfalen, Fachbereich Elektrische Energietechnik und dem Institut für Technologie- und Wissenstransfer im Kreis Soest e.V. durch sein stetes zeitliches und inhaltliches Mitwirken, die vorliegende Arbeit geprägt und aktiv gefördert. Hierfür möchte ich mich ganz herzlich bedanken.

Herrn Prof. Dr. L. Volk, Fachbereich Agrarwirtschaft der Fachhochschule Südwestfalen, gilt mein Dank für die stete Unterstützung und organisatorische Begleitung dieser interdisziplinären Arbeit.

Des Weiteren gilt mein Dank dem Unternehmen Fr. Strube Saatzucht GmbH \& Co. KG, Söllingen, für die Bereitstellung der Mikro-Computertomographieanlage. Frau Dr. A. Wolff 
und ihrem Mitarbeiterteam danke ich für die kompetente Unterstützung und vertrauensvolle Zusammenarbeit.

Abschließend möchte ich meiner Familie, meinen Eltern und ganz besonders meiner Frau Angela danken, dass sie mir auf dem Weg bis hin zur Promotion verständnisvoll und unterstützend zur Seite standen.

Markus Böckelmann 


\section{Lebenslauf}

Name:

Markus Böckelmann

Geburtsdatum:

28.04.1966

Geburtsort:

Hiltrup, jetzt Münster

Familienstand:

verheiratet mit Angela Böckelmann, geb. de Vries, Dipl. -Oecotroph.

Staatsangehörigkeit: deutsch

Schulbildung:

09.1972 - 07.1976

Thomas-Morus-Grundschule, Münster

$08.1976-06.1982$

Wichern-Realschule, Münster, Abschluss: Fachoberschulreife

$08.1982-06.1984$

Ludwig-Erhard-Schule, Münster, Abschluss: Höhere Handelsschule

09. $1984-07.1986$

Ludwig-Erhard-Schule, Münster, Gymnasialer Zweig

Abschluss: Allgemeine Hochschulreife

Ausbildung:

$08.1986-08.1988$

Ausbildung zum Landwirt

Studium:

10.1988 - 07.1992 Studium des Maschinenbaus an der Fachhochschule Münster, Abteilung Steinfurt. Studienabschluss: Diplom-Maschinenbauingenieur (FH)

Zusatzqualifikation: (1993) Ausbildung zum Schweißfachingenieur und an der Schweißtechnischen Lehr- und Versuchsanstalt Duisburg Abschluss: Schweißfachingenieur (EWE)

Tätigkeiten:

03.1993 - 04.1998 Fa. RIELA Getreidetechnik, Riesenbeck. Zunächst als Projektingenieur, ab 01.1995 Bereichsleiter der Konstruktion

Seit 04.1998

Selbständig, Ingenieurbüro

Seit 04.1999

Von der Landwirtschaftskammer Nordrhein-Westfalen öffentlich bestellt und vereidigt als Sachverständiger für Landwirtschaft.

Fachgebiete: Technik in der Landwirtschaft, Innen- und Außenwirtschaft, Klimatechnik und Energiefragen 
Seit 08.1999 Gesamthochschule Paderborn, Abteilung Soest, jetzt Fachhochschule Südwestfalen. Fachbereich Agrarwirtschaft (Prof. Dr. L. Volk) und Elektrische Energietechnik (Prof. Dr. R.- J. Weimar). Studien zu: Prozessoptimierung bei der Trocknung landwirtschaftlicher Produkte.

Seit 03.2003 Externer Doktorand in der Abteilung Agrartechnik des Departments für Nutzpflanzenwissenschaften der Georg-August-Universität Göttingen. 